DR. LAURA FOUASSIER (Orcid ID : 0000-0001-6377-5610)

Article type : Original Article

\title{
ZEB1 promotes cholangiocarcinoma progression through tumor dedifferentiation and tumor-stroma paracrine signaling
}

Cindy Lobe ${ }^{1, *}$, Marie Vallette ${ }^{1, *}$, Ander Arbelaiz ${ }^{1}$, Ester Gonzalez-Sanchez ${ }^{1,2,3}$, Laura Izquierdo ${ }^{3,4}$, Anna Pellat ${ }^{1}$, Nathalie Guedj ${ }^{6}$, Corentin Louis ${ }^{7}$, Valérie Paradis ${ }^{6}$, Jesus M Banales ${ }^{3,4,5}$, Cédric Coulouarn $^{7}$, Chantal Housset ${ }^{1,8}$, Javier Vaquero ${ }^{1,2,3,9, \#, \S}$ \& Laura Fouassier ${ }^{1, \#, \S}$

1-Sorbonne Université, Inserm, Centre de Recherche Saint-Antoine (CRSA), Paris, France. 2-TGF- $\beta$ and Cancer Group, Oncobell Program, Bellvitge Biomedical Research Institute (IDIBELL), Barcelona, Spain.

3-National Biomedical Research Institute on Liver and Gastrointestinal Diseases (CIBERehd), Instituto de Salud Carlos III, Madrid, Spain.

4-Department of Liver and Gastrointestinal Diseases, Biodonostia Health Research Institute Donostia University Hospital, University of the Basque Country (UPV/EHU), San Sebastián, Spain.

5-IKERBASQUE, Basque Foundation for Science, Bilbao, Spain.

6-Service d'Anatomie Pathologique Hôpital Beaujon, F-92110 Clichy, France; INSERM, UMR 1149, Centre de Recherche sur l'Inflammation, F-75018 Paris, France.

7-Inserm UMR 1241, Univ Rennes 1, Rennes, France.

8-Assistance Publique-Hôpitaux de Paris (AP-HP), Saint-Antoine Hospital, Reference center for inflammatory biliary diseases and autoimmune hepatitis, ERN Rare-Liver, Paris, France.

9-LPP, Sorbonne Université, CNRS, Ecole Polytech., Univ. Paris-Sud, Observatoire de Paris, Université Paris-Saclay, PSL Research University, 4 Place Jussieu, 75252 Paris, France

* Share co-first authorship

\# Share co-senior authorship

$\S$ Authors to whom any correspondence should be addressed

Keywords: Cholangiocarcinoma, stroma, cancer-associated fibroblasts, ZEB1, CTGF/CCN2.

This article has been accepted for publication and undergone full peer review but has not been through the copyediting, typesetting, pagination and proofreading process, which may lead to differences between this version and the Version of Record. Please cite this article as doi: 10.1002/hep.32069

This article is protected by copyright. All rights reserved 


\section{Contact information:}

Laura Fouassier, Ph.D.

Sorbonne Université, Faculté de Médecine, Site Saint-Antoine,

27 rue Chaligny, 75571 Paris cedex 12, France.

E-mail: laura.fouassier@inserm.fr Phone: +33-698774001

Javier Vaquero, Ph.D.

TGF-Beta and Cancer Group, IDIBELL,

Gran Via de L'Hospitalet, 199, 08908 Barcelona, Spain.

E-mail: jvaquero@idibell.cat Phone: +34-626569867

List of Abbreviations: cancer-associated fibroblasts (CAF), cancer stem cell (CSC), cholangiocarcinoma (CCA), conditioned media (CM), connective tissue growth factor (CTGF), epidermal growth factor (EGF), epithelial-to-mesenchymal transition (EMT), EMT-inducing transcription factors (EMT-TF), epithelial splicing regulatory protein 1 (ESRP1), extrahepatic CCA (eCCA), hepatic stellate cells (HSC), hepatocyte growth factor (HGF), insulin growth factor 2 (IGF2), interleukin (IL), intrahepatic CCA (iCCA), platelet derived growth factor subunit B (PDGFB), reactive oxygen species (ROS), small hairpin RNAs (shRNAs), the cancer genome atlas (TCGA), transforming growth factor beta (TGF- $\beta$ ), tumour-associated macrophages (TAM).

Financial support: C Lobe is recipient of a fellowship from La Ligue Nationale contre le Cancer (N¹3014). CC is supported by grants from Inserm, Université de Rennes 1, INCa, and ITMO Cancer AVIESAN (Alliance Nationale pour les Sciences de la Vie et de la Santé) dans le cadre du Plan cancer (Non-coding RNA in cancerology: fundamental to translational; [Grant C18007NS]). C Louis is supported by a PhD fellowship from Université de Rennes 1. LF is supported by ANR (ANR-17-CE14-0013-01). AA is supported by the Fondation pour la Recherche Médicale (FRM SPF201809007054) and by Les Entreprises contre le Cancer Paris Île-de-France (GEFLUC) (2019). JV is recipient of the following postdoctoral fellowships from the Spanish Association for the Study of the Liver (AEEH), the Fondation ARC (NPDF2014601431) and the by the LABEXPlas@par project and received financial state aid managed by the Agence Nationale de la Recherche, as part of the programme "Investissements d'avenir" (ANR-11-IDEX0004-02). JV has been funded by Ministerio de Ciencia e Innovación, which is part of Agencia Estatal de Investigación (AEI), through the Retos Investigación grant, numbers RTI2018-094079 and PID2019-108651RJ-I00. JMB is granted by the Spanish Carlos III Health Institute (ISCIII) [(FIS PI15/01132, PI18/01075 and Miguel Servet Program CON14/00129] cofinanced by "Fondo Europeo de Desarrollo Regional" (FEDER), BIOEF (Basque Foundation for Innovation and 
Health Research: EiTB Maratoia BIO15/CA/016/BD), and "Fundación Científica de la Asociación Española Contra el Cáncer" (AECC Scientific Foundation). $\mathrm{CH}$ and LF are supported by the Fondation pour la Recherche Médicale (Equipe FRM 2020 nEQ202003010517). EGS, CC, JMB, JV and LF are members of the European Network for the Study of Cholangiocarcinoma (ENSCCA) and participate to the initiative COST action EURO-CHOLANGIO-NET (CA18122). 
Abstract

\section{Background \& aims}

ZEB1 is a transcription factor that promotes metastatic and stem cell features, which has been associated with poor prognosis in cholangiocarcinoma (CCA), a desmoplastic cancer enriched in cancer-associated fibroblasts (CAF). Here, we aimed to define ZEB1 regulatory functions in malignant and stroma compartments of CCA.

\section{Approach and Results}

Bioinformatic and immunohistochemical analyses were performed to determine correlations between ZEB1 and markers of progressiveness in human intrahepatic CCA (iCCA). Gain/loss of function models were generated in CCA cells, and liver myofibroblasts, as a model of CAF. Conditioned media (CM) was used to unravel tumor-stroma interplay. In vivo experiments were performed using xenograft CCA model. ZEB1 expression in tumor cells of human iCCA was associated with undifferentiated tumor and vascular invasion. In vitro, ZEB1 promoted epithelialmesenchymal transition and stemness in tumor cells leading to cell migration and spheroid formation. In vivo, ZEB1-overexpressing CCA cells formed larger tumors with more abundant stroma. CCN2/CTGF expression was increased in tumor cells from ZEB1-overexpressing xenografts and correlated with ZEB1 expression in human tumors. In vitro, CM from ZEB1overexpressing tumor cells or recombinant CTGF induced myofibroblast proliferation. ZEB1 was also expressed by CAF in human CCA and its expression correlated with CCN2 in myofibroblast and CCA stroma. In mice, co-transplantation of CCA cells with ZEB1-depleted myofibroblasts reduced CCA progressiveness compared to CCA cells/ZEB1-expressing myofibroblasts. Furthermore, ZEB1 controls the expression of paracrine signals (i.e. HGF and IL6) in tumor cells and myofibroblasts.

\section{Conclusions}

ZEB1 plays a key role in CCA progression by regulating tumor cell-CAF cross-talk, leading to tumor dedifferentiation and CAF activation.

This article is protected by copyright. All rights reserved 


\section{1-Introduction}

Cholangiocarcinoma (CCA) includes a heterogeneous group of malignancies that develop from the epithelial layer of the biliary tract. $(1,2)$ CCA is the second most common primary liver tumor after hepatocellular carcinoma, and its incidence is rising worldwide. $(1,2)$ Late diagnosis often compromises surgical resection which is the only effective therapeutic option, that is applicable in only $20 \%$ of cases. Patients ineligible for surgery undergo a palliative treatment with a combination of gemcitabine and cisplatin (GEMCIS).(1-3) High recurrence rates after tumor resection and chemoresistance contribute to the very poor prognosis of CCA. $(1,2)$ Therefore, it is essential to investigate the molecular mechanisms involved in the pathogenesis of CCA, still largely unknown, to identify new potential targets for therapy.

Epithelial-to-mesenchymal transition (EMT) is a dynamic and reversible process by which cells lose epithelial characteristics and acquire mesenchymal properties. $(4,5)$ This process plays a major role in the formation of metastatic carcinoma cells, $(4,5)$ the acquisition of stem cell properties $(4,5)$ and the induction of chemoresistance against a variety of chemotherapeutic drugs and targeted therapies.(4-7) Diverse extracellular signals promote EMT by increasing the expression of EMT-inducing transcription factors (EMT-TF) from the 3 major families: SNAI (SNAIL and SLUG), TWIST (TWIST1 and TWIST2) and ZEB (ZEB1 and ZEB2).(4, 5) ZEB1, that belongs to the Zinc finger E-box binding protein family, is able to recognize E-box sequences in the promoter of certain genes, acting as a transcriptional repressor (i.e. E-cadherin) or activator (i.e. vimentin).(8) In human, ZEB1 becomes aberrantly expressed in different types of cancer, both in tumor and stromal cells, i.e. cancer-associated fibroblasts (CAF)(9), tumor-associated macrophages (TAM),(10) and endothelial cells.(11) Of note, ZEB1 expression in both tumor and stromal cells has been linked to early recurrence and poor prognosis in human pancreatic ductal adenocarcinoma.(12) In this cancer, which displays a strong desmoplastic stroma, ZEB1 expression in CAF supports cancer progression via paracrine signaling with tumor cells.(13) Similarly, CCA is characterized by a dense desmoplastic stroma enriched in CAF that derive either from hepatic stellate cells (HSC) or portal myofibroblasts, and participate in tumor progression $(14,15)$ and chemoresistance.(16)

ZEB1 has been associated with poor prognosis in human intrahepatic CCA (iCCA).(17) Yet, little is known regarding its cellular expression and functions in CCA. Thus, the present study aimed to define the expression of ZEB1 in tumor cells and CAF of CCA and its potential function in the interplay between these two cell types by using preclinical models and human samples of CCA. Here, we describe a new dual function of ZEB1 in CCA. Our data demonstrate that ZEB1 promotes CCA tumor cell dedifferentiation through the acquisition of an EMT/cancer stem cell 
(CSC) phenotype, along with an upregulation of CTGF that contributes to the development of a profibrogenic microenvironment. Moreover, ZEB1 impacts the cross-communication between malignant cells and stromal myofibroblasts by regulating the expression of HGF and IL6, leading ultimately to CCA progression.

This article is protected by copyright. All rights reserved 


\section{2-Materials and methods}

\section{ZEB1 gain and loss of function cell models}

Stable ZEB1 inhibition in SK-ChA-1, HuCCT1 R and in liver myofibroblasts (hTERT-HSC cells) was performed by lentiviral transduction with specific small hairpin RNAs (shRNAs). Plasmids containing sequences targeting ZEB1 (shRNA-ZEB1) or scramble (shRNA-Control) designed by GenecopoeiaTM (Maryland,USA) were used to produce $\triangle \mathrm{U} 3$ SIN lentivirus in the Viral and Gene Transfer Vectors Platform of Necker (Paris, France). Briefly, cells were infected and maintained in presence of lentivirus for $24 \mathrm{~h}$. Then, lentiviruses were removed, and fresh media was added to maintain cells in culture another $48 \mathrm{~h}$ until mCherry expression was observed. Positive transduced cells were detected by positive fluorescence signal and they were selected by treatment with lethal doses of puromycin for $72 \mathrm{~h}$ to eliminate non-infected cells. Due to incomplete efficiency of puromycin selection in SK-ChA-1 and HuCCT1 R, 2 clones of each cell type were isolated and characterized throughout the study. The cell transduction was performed with level 2 precautions according to biosafety regulation standard ISO 15190:2003.

ZEB1 experimental overexpression was performed by retroviral infection. Plasmids coding for the murine open reading frame of Zeb1 or Mock (control), were used to produce retrovirus in the same platform, following the protocol previously described by Dr. Julie Caramel (Cancer Research Center, Lyon, France).(18) For retroviral transduction, the same protocol was applied to the HuCCT1 cells. In this case, puromycin was effective and cell populations were used in the study.

HuCCT1 resistant cells to erlotinib (HuCCT1 R) were generated through a process of slowly escalating exposure to erlotinib (from 1 to $20 \mu \mathrm{M}$ ), an EGFR tyrosine kinase inhibitor, as previously described.(16)

For clarification purposes, along the manuscript cell lines or murine models derived from injection of cells with low ZEB1 expression will be represented by white bars, while those with high ZEB1 expression will be represented by black bars.

Further information about the materials and methods is available in the Supplementary Information. 


\section{3-Results}

3.1- ZEB1 expression in malignant cells is associated with tumor differentiation and vascular invasion in human CCA

To examine ZEB1 expression, we performed an immunostaining in a set of tissue samples from 45 subjects with iCCA. While ZEB1 expression was absent from cholangiocytes of adjacent liver tissue (Figure 1A, yellow arrow), 20\% of iCCA displayed a nuclear expression of ZEB1 in cancer cells (Figure 1A, black arrows). ZEB1 was also observed in stromal cells (Figure 1A, red arrows). In cancer cells, ZEB1 expression positively associated with tumor differentiation (Figure 1B) and vascular invasion (Figure 1C). Accordingly, the expression of ZEB1 in a panel of 8 human CCA cell lines (including iCCA and extrahepatic CCA (eCCA) cell lines) was inversely correlated with the expression of E-cadherin and ESPR1, two epithelial markers, and positively with that of vimentin, a mesenchymal marker, at protein (Figure 1D) and mRNA levels (Supplementary Figure 1). Furthermore, ZEB1 is the EMT-TFs that best correlated both negatively with the expression of epithelial markers and positively with the mesenchymal marker (Supplementary Figure 1). Interestingly, CCA cell lines with negligible ZEB1 expression are derived from more differentiated human tumors, than the cell lines with high ZEB1 expression that were obtained from undifferentiated tumors.(19-24) Accordingly, transcriptomic expression analyses showed a strong negative correlation between ZEB1 and both $C D H 1$ (encoding E-cadherin) and ESRP1 in human CCA from Rennes and TCGA cohorts, and a strong positive correlation between ZEB1 and VIM (encoding vimentin) in both datasets (Figure 1E).

\section{2- ZEB1 regulates CCA cell dedifferentiation by inducing epithelial-to-mesenchymal transition and stemness phenotype}

To decipher the molecular mechanisms by which ZEB1 regulates the differentiation of cancer cells, we modulated ZEB1 expression in CCA cells by different approaches. In HuCCT1 cells, the murine form of Zeb1 was overexpressed as previously described,(18) and, in SK-ChA-1 cells, ZEB1 was downregulated by shRNA (Figure 2A-C). Additionally, HuCCT1 cells resistant to erlotinib (HuCCT1-R, previously generated in our laboratory),(16) which overexpressed ZEB1 as a cause of resistance, were also modified to downregulate ZEB1 (Figure 2A-C). In the 3 cellular models, genetic manipulation of ZEB1 did not induce a compensatory alteration at the mRNA or protein level of other EMT-TFs (Supplementary Figure 2). Interestingly, ectopic expression of ZEB1 in HuCCT1 induced a decrease of the epithelial markers CDH1 and ESRP1 and an upregulation of the mesenchymal marker VIM (Figure 3A-C), whereas ZEB1 downregulation had opposite effects in SK-ChA-1 and HuCCT1-R (Supplementary Figures 3A-C and 4A-C). Because

This article is protected by copyright. All rights reserved 
EMT-TFs are linked to the acquisition of stemness features,(8) we evaluated the impact of ZEB1 in the regulation of two CSC markers, CD44 and CD24, allowing the quantification of a population (CD44 ${ }^{\text {high/CD24low })}$ that shares EMT/CSC traits. $(16,25)$. Accordingly, overexpression of ZEB1 caused an upregulation of CD44 and a marked downregulation of CD24 (Figure 3D), whereas ZEB1 knockdown had the opposite effect (Supplementary Figures 3D and 4D). These data were confirmed by flow cytometry analysis, indicating that high ZEB1 expression concurred with an increase of CD44high/CD24low cell population (Figure 3E) and inversely when ZEB1 is downregulated (Supplementary Figures $3 \mathrm{E}$ and $4 \mathrm{E}$ ).

These phenotypic modifications were functionally translated by an increased migration, colony and sphere formation in HuCCT1 Zeb1-overexpressing cells (Figure $3 \mathrm{~F}-\mathrm{H}$ ) and by a mirroring effect in SK-ChA-1 and HuCCT1-R cells downregulated for ZEB1 (Supplementary Figures 3F-H and $4 \mathrm{~F}-\mathrm{H}$ ), compared with the corresponding controls. Altogether, these observations indicate that ZEB1 expression in CCA cells confers high plasticity leading to cell dedifferentiation.

\section{3- ZEB1 induces the generation of larger CCA tumors with abundant stromal content}

To determine the biological relevance of ZEB1 in CCA cells, we generated CCA tumors into immunocompromised mice by engrafting cancer cells that overexpressed ZEB1 (HuCCT1-Zeb1). CCA cells overexpressing Zeb1 generated larger tumors (Figure 4A-B), histologically characterized by a prominent desmoplastic stroma (Figure 4C) with higher collagen deposition (Figure 4D), compared with control tumors. In this model, engraftment of human cancer cells gave rise to tumors with murine stromal cells, which we took advantage of to analyze gene expression in cancer cells and stromal cells using human and murine specific primers, respectively. In this context, the expression of murine Acta2 ( $\alpha-S m a)$, a marker of CAF, and Col1a1 (collagen 1) and Col4a1 (collagen 4), extracellular matrix (ECM) components produced by CAF, was increased in the stroma of tumors developed from CCA cells overexpressing Zeb1 (HuCCT1-Zeb1) (Figure 4E) in agreement with the histological observations (Figure 4C-D). These observations agreed with our previous findings in resistant CCA tumors (developed from HuCCT1-R cells that overexpress ZEB1), in which the stroma content was also increased.(16) To understand how the stromal compartment expanded upon transplantation of Zeb1overexpressing tumor cells (HuCCT1-Zeb1), we evaluated the expression of key pro-fibrogenic factors, TGFB1 (encoding transforming growth factor beta 1, TGF- $\beta 1$ ), CCN2 (encoding connective tissue growth factor, CTGF) and PDGFB (encoding Platelet Derived Growth Factor Subunit B, PDGFB) in the tumoral human compartment, corresponding to CCA cells. The expression of TGFB1 and PDGFB in CCA cells, was unchanged in tumors generated from the cells overexpressing Zeb1, whereas a significant increase in CCN2 mRNA levels was observed 
(Figure 4F). The analysis of resistant CCA tumor samples overexpressing ZEB1, obtained from our previous work(16) revealed an even stronger increase in CCN2 expression, compared to WT (Figure 4G). Protein analyses confirmed the upregulation of CTGF expression in ZEB1 overexpressing tumors (Figure $4 \mathrm{H}-\mathrm{I}$ ). These results suggest that in tumor cells, ZEB1 positively regulates CCN2/CTGF, which boosts the stromal expansion of CCA.

\section{4- ZEB1 regulates CCN2 transcription in CCA cells leading to myofibroblast expansion}

The transcriptional activation of CCN2/CTGF by ZEB1 has been described in breast cancer cells through its cooperation with YAP signaling,(26) which is active in CCA cells.(27) Accordingly, analysis of several CCA cell lines showed that CCN2 expression correlated with ZEB1 expression (Supplementary Figure $5 A$ ). Evaluation of genetically manipulated CCA cells in which ZEB1 is either overexpressed or down-regulated, demonstrated that the mRNA expression of CCN2 concurred with that of ZEB1, showing a strong upregulation of CCN2 expression in Zeb1overexpressing cells (HuCCT1-Zeb1) and a downregulation in ZEB1-depleted CCA cells (SKChA-1- and HuCCT1 R-shZEB1 cells), compared with controls (Figure 5A). At the protein level, CTGF expression also concurred with ZEB1 in HuCCT1 Control/Zeb1 and HuCCT1 R-shRNA cell lines (Figure 5A). Despite mRNA level difference in SK-ChA-1, there were no differences of CTGF at the protein level (Figure 5A), hypothesizing the existence of unknown posttranslational mechanisms in this cell line. Regarding TGFB1 expression, no differences were observed (Supplementary Figure 5B) corroborating our in vivo findings (Figure 4F and 4G). Transcriptomic analysis of human CCA showed a strong positive correlation between CCN2 and ZEB1 expressions in the Rennes Cohort and a similar trend in the TCGA cohort (Figure 5B). To evaluate the effect of CTGF on stromal cells, human liver myofibroblasts (hTERT-HSC cell line), used as a model of CAF, were exposed to recombinant CTGF (Figure $5 \mathrm{C}$ ) or to conditioned media (CM) from Zeb1-overexpressing cells (HuCCT1-Zeb1) in which CCN2 is upregulated (Figure 5D). In both cases, the proliferation of myofibroblasts was increased, compared with the controls (Figure 5C-D), indicating a pro-proliferative effect of CTGF on myofibroblasts, consistent with its role in hepatic fibrosis. $(28,29)$ Furthermore, addition of a neutralizing antibody against CTGF to CM of HuCCT1-Zeb1 reduces significantly the effect on myofibroblasts viability (Figure 5D). Contrarily, CTGF had no effect on CCA cell proliferation (Supplementary Figure 5C) nor on the expression of EMT markers and pro-inflammatory cytokines (Supplementary Figure 5D). Finally, ChIP analyses demonstrated that ZEB1 upregulates CCN2 expression by directly binding its promoter (Figure 5E), as previously demonstrated in breast cancer.(26)

Finally, to extend our findings on the relationship between ZEB1 and CCN2 to other cancer types, ZEB1 and CCN2 expression was analyzed both in human cell lines and tissues using the 
Broad-Novartis Cancer Cell Line Encyclopaedia database that includes 1156 cell lines (Supplementary Figure 6A) and the TCGA datasets (Supplementary Figure 6B). We confirmed that ZEB1 and CCN2 are interrelated in other cancers, suggesting a ubiquitous mechanism of regulation.

\section{5- ZEB1 is expressed by CAF and regulates their activation status}

CCA is characterized by a dense desmoplastic stroma rich in CAF, which have an important role in tumor progression.(14) Our observation of ZEB1 expression in stromal cells from CCA patients (Figure 1A, red arrows), led us to further investigate the role of ZEB1 in CAF. First, immunostaining of ZEB1 and $\alpha$-SMA in human iCCA samples, showed a co-expression of both proteins in stromal areas (Figure 6A, red arrows), indicating that $\alpha$-SMA expressing CAF also express ZEB1. Of note, some stromal cells showed ZEB1 expression in absence of $\alpha$-SMA (Figure 6A, black arrows), suggesting that other stromal populations may express ZEB1. Furthermore, transcriptomic analysis of laser microdissected tissue from stromal areas of human iCCA showed a strong correlation of ZEB1 with ACTA2 and COL4A1, and with the pro-fibrotic factors TGFB1 and CCN2 (Figure 6B), but not with PDGFB (Supplementary Figure 7A). Accordingly, analysis of xenografts derived from ZEB1-overexpressing CCA cells (HuCCT1-Zeb1 and HuCCT1-R), which display a more prominent and activated stroma, showed an upregulation of Zeb1 in stroma (i.e. mouse gene, Figure 6C-D) together with that of Acta2, Col4A1 (Figure 4E and ref (16)), Tgfb1, Ccn2 and Pdgfb (Supplementary Figure $7 \mathrm{~B}-\mathrm{C}$ ). To validate in vitro the regulation of these factors by ZEB1, ZEB1 was stably downregulated in hepatic myofibroblasts (Figure 6E), resulting in decreased expression of TGFB1, CCN2, PDGFB, ACTA2 and COL4A1, (Figure $6 \mathrm{~F}$ ). ChIP analyses confirmed the direct regulation of CCN2 by ZEB1 also in human liver myofibroblasts (Supplementary Figure 7D). Downregulation of CCN2 in hTERT-HSC by siRNA was insufficient to modify the expression of TGFB1, PDGFB and COL4A1 and only a small although significant reduction of ACTA2 was observed in cells (Supplementary Figure 7E), indicating that ZEB1 effects are not dependent on CCN2 expression or at least not exclusively. Altogether, these results provide evidence of the role of ZEB1 in the activation of stromal CAF.

This article is protected by copyright. All rights reserved 


\subsection{Depletion of ZEB1 in myofibroblasts reduces CCA progressiveness}

Next, we evaluated the in vivo relevance of ZEB1 expression in human liver myofibroblasts on CCA growth. CCA cells were co-engrafted with liver myofibroblasts depleted for ZEB1 (hTERTHSC-shZEB1) or not (hTERT-HSC-shControl). We used EGI-1 CCA cells because they have negligible expression of ZEB1 (to avoid interference with ZEB1 expressed by stromal cells) and their tumorigenic potential is the highest among our CCA cell lines. Transplantation of CCA cells with liver myofibroblasts depleted for ZEB1 generated smaller tumors compared to the controls (Figure 7A). One concern of transplanting human liver myofibroblasts in immunocompromised mouse is their replacement by resident mouse fibroblasts during tumorigenesis. Indeed, mRNA expression of fibroblast related genes from human origin (ACTA2, COL1A1 and COL4A1) was very low in the tumors (Supplementary Figure $8 \mathrm{~A}$ ) and similar to that of xenografts derived from implantation of EGI-1 cells alone.(16) Interestingly, analysis of the murine counterparts showed a downregulation of Zeb1, Tgfb1, Acta2, Col4a1, and a trend for Ccn2 and Pdgfb expression in the stromal compartment of tumors developed from CCA cells with ZEB1-depleted myofibroblasts, compared with the controls (Figure 7B), indicating that, in addition of promoting tumor growth, human myofibroblasts could educate the recruited murine stroma at an early time point. On the contrary, no changes in profibrotic factors were observed in the tumor compartment (Supplementary Figure 8B).

Finally, to evaluate the direct effect of liver myofibroblasts on tumor cells, CCA cells were exposed to CM from ZEB1-depleted myofibroblasts or the controls and CCA cell viability was analyzed. CM from control myofibroblasts (hTERT-HSC-shRNA-Control) strongly increased the viability of EGI-1 and HuCCT1 cells, compared with serum-free media. In contrast, CM from ZEB1-depleted myofibroblasts showed a weak effect on the viability of EGI-1 cells and no effect on HuCCT1 cells (Figure 7C). Changes in cell viability were accompanied by the activation of pro-survival intracellular signaling pathways. CM from control myofibroblasts activated STAT3 and AKT in CCA cells, while CM from ZEB1-depleted myofibroblasts had very low or no effect on these signaling pathways (Figure 7D). No differences were observed in the status of ERK1/2 (Figure 7D). No changes in mRNA level of EMT markers and pro-inflammatory cytokines were observed in CCA cells treated with any of the CM from myofibroblasts (Supplementary Figure 9). Altogether, these results suggest that ZEB1 in myofibroblasts regulates their interplay with CCA cells, that depends on growth factors secreted by CAF.

\section{7- ZEB1 impacts the interplay between CCA tumor cells and stromal myofibroblasts}

This article is protected by copyright. All rights reserved 
Our study suggests that ZEB1 regulates the interplay between CCA cells and CAF to promote CCA progression. Therefore, as a proof of concept, we selected four molecules with major roles in CCA progression that could play a role in ZEB1-regulated interplay: insulin growth factor 2 (IGF2),(16) epidermal growth factor (EGF),(30) hepatocyte growth factor (HGF)(31) and interleukin 6 (IL6).(32) We analyzed the expression of these factors in our preclinical models, including i/ Zeb1-overexpressing cells (HuCCT1-Zeb1 cells vs control cells) and mouse CCA tumors generated from these cells, ii/ CCA cells resistant to erlotinib that overexpressed ZEB1 (HuCCT1-R vs WT cells) and tumors generated from these cells, and iii/ ZEB1-depleted myofibroblasts (hTERT-HSC-shZEB1 vs control cells) and tumors generated from EGI-1 cells with myofibroblasts (Figure 8 and Supplementary Figure 10).

Both IGF2 and EGF expression increased in ZEB1-overexpressing cells (HuCCT1-Zeb1 and HuCCT1 R) compared with their controls (Supplementary Figure 10A-B). Similarly, in ZEB1depleted myofibroblasts, IGF2 and EGF expression was strongly downregulated compared to the controls (Supplementary Figure 10A-B). However, once implanted in mice no change was observed in the tumor (Supplementary Figure 10A-B), suggesting that the complexity of in vivo systems may influence ZEB1 regulation of these factors.

HGF was found overexpressed in Zeb1-overexpressing cells (HuCCT1-Zeb1) and resistant cells (HuCCT1-R) compared to the controls (Figure 8A). Interestingly, in vivo HGF was overexpressed in both human (reflecting expression in cancer cells) and murine (reflecting expression in stromal cells) compartments of CCA tumors (Figure 8A). On the contrary, no change in the expression of HGF was observed in cultured myofibroblasts and in vivo in the co-transplantation xenograft model (Figure 8A). The increased HGF expression in the stroma of mice tumors derived from ZEB1-overexpressing HuCCT1 cells suggested that ZEB1 in malignant cells induces HGF expression in stromal cells through paracrine signal. To test this hypothesis, we exposed liver myofibroblasts (hTERT-HSC) to CM from Zeb1-overexpressing cells (HuCCT1-Zeb1) or the controls. Indeed, CM from HuCCT1-Zeb1 cells upregulated HGF expression in myofibroblasts compared to control CM (Figure 8B). However, neither direct treatment with exogenous CTGF nor the addition of a neutralizing antibody against CTGF to the CM of HuCCT1-Zeb1 cells modified the HGF expression in hTERT-HSC cells, indicating that other factors upregulate HGF (Supplementary Figure 11A-B). Transcriptomic analysis of human CCA showed a strong and positive correlation between ZEB1 and HGF expressions in both the Rennes and the TCGA cohorts, which was confirmed in other cancer types using public data bases (Supplementary Figure 12A-C). Further ChIP assays demonstrated the direct binding of ZEB1 to HGF promoter (Supplementary Figure 12D).

This article is protected by copyright. All rights reserved 
The only statistical change observed for IL6 was a strong downregulation in myofibroblasts depleted for ZEB1 (hTERT-HSC-shZEB1) compared to controls, that was also observed in the stromal compartment of CCA xenografts (Figure $8 \mathrm{C}$ ). This specific regulation of IL6 in the liver myofibroblasts suggested that ZEB1 may regulate IL6 in CAF but not in malignant cells. To address this possibility, we treated ZEB1-depleted myofibroblasts (hTERT-HSC-shZEB1) or the controls with TGF- $\beta$, the classical molecule used to activate myofibroblasts and we monitored the expression of ZEB1 and IL6 genes. ZEB1 expression was upregulated in response to TGF- $\beta$ in control myofibroblasts and as expected, this response was completely abolished in ZEB1depleted cells, confirming the efficiency of our shRNA system (Figure 8D). In agreement with our hypothesis, IL6 response overlapped with that of ZEB1, being upregulated by TGF- $\beta$ in control myofibroblasts, but abolished in cells depleted for ZEB1, confirming ZEB1 regulation of IL6 in liver myofibroblasts (Figure 8D). In human CCA, IL6 showed a strong correlation with ZEB1 expression in both the Rennes and the TCGA cohorts (Supplementary Figure 13A). Similarly, this correlation was confirmed in other human cancers from the Broad-Novartis Cancer Cell Line Encyclopaedia and the TCGA datasets (Supplementary Figure 13B-C).

To functionally translate the importance of ZEB1 regulation over these factors, we performed viability studies on a panel of malignant cells and liver myofibroblasts (including those in which ZEB1 expression was genetically modified) by exposing the cells to HGF and IL6. These experiments show that HGF was able to increase the viability of almost all cell lines tested (Figure 8E), while IL6 was only able to potentiate the viability of HuCCT1 cells (both HuCCT1 Control and Zeb1) (Figure 8F). Nevertheless, the most interesting effect observed was that the modulation of ZEB1 expression leads to increase (in HuCCT1-Zeb1) or reduce (in hTERT-HSCshZEB1) response to HGF (Figure 8E). A similar ZEB1-dependent modulatory response was observed when HuCCT1-Control and Zeb1 cells were exposed to CM of hTERT-HSC, and when hTERT-HSC-shControl and shZEB1 were exposed to CM of HuCCT1 (Supplementary Figure 14A-B). Furthermore, HGF promotes a moderate activation of hTERT-HSC-shControl cells, but has no effect in hTERT-HSC depleted for ZEB1 (Supplementary Figure 14C). However, mRNA expression of receptors for HGF (MET) and IL6 (IL6R and IL6ST, encoding GP130) did not explain the altered sensitivity to these soluble factors (Supplementary Figure 15A-C), indicating that modifications of other signaling mediators account for these effects.

Altogether, these results indicate that ZEB1 regulates not only the production of soluble signaling molecules (such as HGF) but their signaling pathways creating a signaling hub between tumor and stromal cells to boost CCA growth.

This article is protected by copyright. All rights reserved 


\section{4 -Discussion}

The final purpose of translational research is to extrapolate findings from preclinical models into new therapeutic targets, which are potentially targetable in clinical practice to improve patient management, in this case CCA patients. Following this strategy, we have used multiple cell culture models, animal experimentation with tumor xenografts and validated our findings by taking advantage of immunostaining and transcriptomic data from human CCA to pinpoint ZEB1 as an ideal therapeutic candidate against CCA for which new ways of treatment should be further explored.

ZEB1 is a transcription factor originally discovered as a mediator of the EMT process in embryonic development.(33) During the last decades it has acquired a prominent role in the biology of tumor cells in a variety of cancers by promoting tumor cell stemness, invasion and chemoresistance to an assortment of drugs.(8) Here we confirm a role for ZEB1 in the dedifferentiation and the acquisition of a more aggressive phenotype of CCA tumor cells. ZEB1 increased cell plasticity through modulation of epithelial (i.e. E-cadherin), mesenchymal (i.e. vimentin) and stemness (i.e. CD44/CD24) marker expression, providing them with migratory and stemness properties, which in human CCA was translated as a correlation of ZEB1 expression in tumor cells with poorly differentiated tumors and vascular invasion. These data open the way of the potential therapeutic usefulness of targeting ZEB1 in late stages of CCA, which could prevent cell dissemination by impairing EMT, and might have a profound impact in reducing cancer progression.

Recent evidence points to ZEB1 involvement in different aspects of tumor progression through its expression in several stromal cell types, $(9-11,13)$ although ZEB1 role in these cells is not yet fully understood. The desmoplastic nature of CCA stroma led us to focus a large part of our study on demonstrating the role of ZEB1 in the activation of stromal CAF. Nevertheless, we also found ZEB1 in areas of human iCCA that resemble tumor infiltrates (Supplementary Figure 16A) and vascular structures (Supplementary Figure 16B), indicating that ZEB1 is probably present also in immune cells and endothelial cells of CCA. Accordingly, major functions for ZEB1-expressing TAM and endothelial cells have been described in other cancers. $(10,11)$ This observation opens the door to further research to fully understand ZEB1 regulatory network over the different cell types present in CCA and how they interact to each other.

We also provide evidence that ZEB1 is at the interface of tumor-stroma cross-communication by regulating the expression of growth factors and proinflammatory cytokines that impact CCA progression.

This article is protected by copyright. All rights reserved 
CCN2/CTGF is a growth factor with a prominent role in the activation of HSC during liver fibrosis.(29) In accordance with previous observations that place ZEB1 as a regulator of CTGF,(26) we show that ZEB1 is an inducer of CCN2 expression at both sides of the CCA cellCAF crosstalk by direct binding to its promoter. Modulation of ZEB1 resulted in a concomitant change of CCN2 expression in all genetically modified tumor and myofibroblast cells, even when these cell lines were implanted into immunocompromised mice to generate tumors. Moreover, ZEB1 and CCN2 expression correlated in human samples, translating our preclinical evidence into iCCA. Interestingly, our data suggest that this regulation occurs in both human and murine species, as shown by the ability of both murine (HuCCT1-Zeb1) and human (HuCCT1 R) ZEB1 to upregulate CCN2 in CCA cells. These observations show the high degree of evolutionary conservation of this regulatory mechanism, depicting the biological importance of ZEB1-CCN2 tandem in CCA and probably in other pathologies (including several types of cancer) as previously described $(34,35)$ and the strong correlation found in cancers from the TCGA cohort. Furthermore, CCN2 has been implicated in promotion of inflammation and angiogenesis(35) and preliminary analyses of specific macrophage and endothelial cell markers in our in vivo models (Supplementary Figure $16 \mathrm{C}-\mathrm{D}$ ), suggest that ZEB1 expression in tumor cells may have a more important role in the reprograming of entire stromal compartment through CCN2/CTGF in late stages of cancer to support tumor growth and dissemination. These evidences together with the fact that CTGF is an independent prognostic factor indicator of both tumor recurrence and overall survival for intrahepatic $\mathrm{CCA}(36)$ ensures its potential as a target for the future of this disease. In this sense, Pamrevlumab, a CTGF neutralizing antibody, recently reached the clinics and results from trials in pancreatic cancer are encouraging,(37) showing that the use of Pamrevlumab in combination with gemcitabine and nab-paclitaxel has potential to enhance tumor response and increase resection rates. Looking at the similarities of pancreatic cancer and CCA, both exhibiting a high degree of desmoplasia due to extensive extracellular matrix deposition, once more the evidence points to the potential use of the anti-CTGF antibody in CCA.

We also found that ZEB1-overexpressing tumor cells displayed an elevated expression of HGF, in both cells and murine preclinical models of CCA, and direct binding of ZEB1 to HGF promoter was demonstrated by CHIP analysis for the first time to our knowledge. Furthermore, ZEB1 expression in CCA tumor cells induced an upregulation of HGF in stromal cells from xenografts and CM from ZEB1-overexpressing cells increased HGF expression in myofibroblasts in vitro. A recent work describes a similar paracrine regulatory mechanism by which ZEB1 expression in tumor cells is able to stimulate stromal macrophage production of signaling molecules (i.e. ROS and IL-1ß) to promote inflammatory colorectal carcinoma.(38) Nonetheless, the exact paracrine 
signal that favors HGF production by CAF in CCA is yet to be identified. Interestingly, we also observed that manipulation of ZEB1 expression in CCA cells and fibroblasts correlated with the ability of these cells to respond to exogenous HGF in vitro. Since expression of the main HGF receptor (MET) did not explain this ZEB1-dependent response, we hypothesized that ZEB1 may be modulating the expression of other mediators that could influence HGF signaling. MET has been described as a promiscuous receptor able to interact with multiple partners that act as coreceptors, modulating its signaling.(39) Among them, integrins are membrane receptors described to play important roles in the establishment of EMT.(4) Previous results from our group(16) led us to hypothesize a potential implication of Integrin Beta 3 (ITGB3, also known as CD61), which has also been described as a CTGF receptor in fibroblasts.(40) Indeed, expression analysis indicate that mRNA and protein expression of ITGB3 perfectly correlated with that of ZEB1 in HuCCT1 and hTERT-HSC modified cells (Supplementary Figure 17A-B). Although very preliminary data, further functional experiments are guaranteed to unravel the exact functions of ITGB3 downstream of ZEB1.

The tumor microenvironment plays a role in cancer progression from initiation to metastasis and therapeutic response.(41) Stromal CAF from CCA are known to produce a wide variety of growthstimulatory, pro-angiogenic, immune-modulatory and pro-invasive factors, as well as different ECM components.(42) IL6 binds to specific receptors on tumor and stromal cells leading to cancer progression.(43) It has been demonstrated that IL 6 and IL8 are directly regulated by ZEB1 in breast cancer cells,(44) but we did not find any relationship between ZEB1 and IL6 expression in CCA tumor cells. Here we found that ZEB1 invalidation in fibroblasts reduces the expression of IL6 to the surrounding stroma. Recently it has been described that IL6 can be indirectly regulated by ZEB1 through a mechanism that involves p53 acetylation and recruitment to IL6 promoter in stromal fibroblast from tumor of mammary origin.(9) Intriguingly, we observed a similar regulation of both IL6 and CXCL8 (IL8) in hTERT-HSC invalidated for ZEB1 and exposed to TGF- $\beta 1$ (Figure $8 \mathrm{D}$ and Supplementary Figure 18), although the absence of a murine IL8 ortholog made impossible its analysis in the stroma of xenografts. The in vitro experiments showed a modest effect of IL6 on CCA cell lines viability. However, effects of IL6 at other levels of the tumorigenic process (43) remain to be explored in our experimental models.

In conclusion, our results highlight ZEB1 as a key player in the progression of CCA, not only by allowing tumor cells to become more dedifferentiated and aggressive, by participating in the activation of CAFs and the production of extracellular matrix, but also through regulation of crosstalk signals that boost CCA growth (summarized in Figure 8G).

This article is protected by copyright. All rights reserved 


\section{References}

1. Banales JM, Cardinale V, Carpino G, Marzioni M, Andersen JB, Invernizzi P, Lind GE, et al. Expert consensus document: Cholangiocarcinoma: current knowledge and future perspectives consensus statement from the European Network for the Study of Cholangiocarcinoma (ENS-CCA). Nat Rev Gastroenterol Hepatol 2016;13:261-280.

2. Banales JM, Marin JJG, Lamarca A, Rodrigues PM, Khan SA, Roberts LR, Cardinale V, et al. Cholangiocarcinoma 2020: the next horizon in mechanisms and management. Nat Rev Gastroenterol Hepatol 2020.

3. Valle J, Wasan H, Palmer DH, Cunningham D, Anthoney A, Maraveyas A, Madhusudan S, et al. Cisplatin plus gemcitabine versus gemcitabine for biliary tract cancer. N Engl J Med 2010;362:1273-1281.

4. Pastushenko I, Blanpain C. EMT Transition States during Tumor Progression and Metastasis. Trends Cell Biol 2019;29:212-226.

5. Vaquero J, Guedj N, Claperon A, Nguyen Ho-Bouldoires TH, Paradis V, Fouassier L. Epithelial-mesenchymal transition in cholangiocarcinoma: From clinical evidence to regulatory networks. J Hepatol 2017;66:424-441.

6. Tulchinsky E, Demidov O, Kriajevska M, Barlev NA, Imyanitov E. EMT: A mechanism for escape from EGFR-targeted therapy in lung cancer. Biochim Biophys Acta Rev Cancer 2019;1871:29-39.

7. van Staalduinen J, Baker D, Ten Dijke P, van Dam H. Epithelial-mesenchymal-transitioninducing transcription factors: new targets for tackling chemoresistance in cancer? Oncogene 2018;37:6195-6211.

8. Caramel J, Ligier M, Puisieux A. Pleiotropic Roles for ZEB1 in Cancer. Cancer Res 2018;78:30-35.

9. Fu R, Han CF, Ni T, Di L, Liu LJ, Lv WC, Bi YR, et al. A ZEB1/p53 signaling axis in stromal fibroblasts promotes mammary epithelial tumours. Nat Commun 2019;10:3210.

10. Cortes M, Sanchez-Moral L, de Barrios O, Fernandez-Acenero MJ, Martinez-Campanario MC, Esteve-Codina A, Darling DS, et al. Tumor-associated macrophages (TAMs) depend on ZEB1 for their cancer-promoting roles. EMBO J 2017;36:3336-3355. 
11. Fu R, Li Y, Jiang N, Ren BX, Zang CZ, Liu LJ, Lv WC, et al. Inactivation of endothelial ZEB1 impedes tumor progression and sensitizes tumors to conventional therapies. J Clin Invest 2020;130:1252-1270.

12. Bronsert $P$, Kohler I, Timme S, Kiefer S, Werner M, Schilling O, Vashist $Y$, et al. Prognostic significance of Zinc finger E-box binding homeobox 1 (ZEB1) expression in cancer cells and cancer-associated fibroblasts in pancreatic head cancer. Surgery 2014;156:97-108.

13. Sangrador I, Molero X, Campbell F, Franch-Exposito S, Rovira-Rigau M, Samper E, Dominguez-Fraile $\mathrm{M}$, et al. Zeb1 in Stromal Myofibroblasts Promotes Kras-Driven Development of Pancreatic Cancer. Cancer Res 2018;78:2624-2637.

14. Cadamuro M, Brivio S, Mertens J, Vismara M, Moncsek A, Milani C, Fingas C, et al. Plateletderived growth factor- $D$ enables liver myofibroblasts to promote tumor lymphangiogenesis in cholangiocarcinoma. J Hepatol 2019;70:700-709.

15. Claperon A, Mergey M, Aoudjehane L, Ho-Bouldoires TH, Wendum D, Prignon A, Merabtene $F$, et al. Hepatic myofibroblasts promote the progression of human cholangiocarcinoma through activation of epidermal growth factor receptor. Hepatology 2013;58:2001-2011.

16. Vaquero J, Lobe C, Tahraoui S, Claperon A, Mergey M, Merabtene F, Wendum D, et al. The IGF2/IR/IGF1R Pathway in Tumor Cells and Myofibroblasts Mediates Resistance to EGFR Inhibition in Cholangiocarcinoma. Clin Cancer Res 2018;24:4282-4296.

17. Terashita K, Chuma M, Hatanaka Y, Hatanaka K, Mitsuhashi T, Yokoo H, Ohmura T, et al. ZEB1 expression is associated with prognosis of intrahepatic cholangiocarcinoma. $\mathrm{J}$ Clin Pathol 2016;69:593-599.

18. Caramel J, Papadogeorgakis E, Hill L, Browne GJ, Richard G, Wierinckx A, Saldanha G, et al. A switch in the expression of embryonic EMT-inducers drives the development of malignant melanoma. Cancer Cell 2013;24:466-480.

19. Knuth A, Gabbert H, Dippold W, Klein O, Sachsse W, Bitter-Suermann D, Prellwitz W, et al. Biliary adenocarcinoma. Characterisation of three new human tumor cell lines. $J$ Hepatol 1985;1:579-596.

20. Kusaka Y, Tokiwa T, Sato J. Establishment and characterization of a cell line from a human cholangiocellular carcinoma. Res Exp Med (Berl) 1988;188:367-375.

21. Miyagiwa M, Ichida T, Tokiwa T, Sato J, Sasaki H. A new human cholangiocellular carcinoma cell line (HuCC-T1) producing carbohydrate antigen 19/9 in serum-free medium. In Vitro Cell Dev Biol 1989;25:503-510. 
22. Saijyo S, Kudo T, Suzuki M, Katayose $\mathrm{Y}$, Shinoda $\mathrm{M}$, Muto $\mathrm{T}$, Fukuhara $\mathrm{K}$, et al. Establishment of a new extrahepatic bile duct carcinoma cell line, TFK-1. Tohoku J Exp Med 1995;177:61-71.

23. Shimizu Y, Demetris AJ, Gollin SM, Storto PD, Bedford HM, Altarac S, Iwatsuki S, et al. Two new human cholangiocarcinoma cell lines and their cytogenetics and responses to growth factors, hormones, cytokines or immunologic effector cells. Int J Cancer 1992;52:252-260.

24. Storto PD, Saidman SL, Demetris AJ, Letessier E, Whiteside TL, Gollin SM. Chromosomal breakpoints in cholangiocarcinoma cell lines. Genes Chromosomes Cancer 1990;2:300-310.

25. Mani SA, Guo W, Liao MJ, Eaton EN, Ayyanan A, Zhou AY, Brooks M, et al. The epithelialmesenchymal transition generates cells with properties of stem cells. Cell 2008;133:704-715.

26. Lehmann W, Mossmann D, Kleemann J, Mock K, Meisinger C, Brummer T, Herr R, et al. ZEB1 turns into a transcriptional activator by interacting with YAP1 in aggressive cancer types. Nat Commun 2016;7:10498.

27. Marti P, Stein C, Blumer T, Abraham Y, Dill MT, Pikiolek M, Orsini V, et al. YAP promotes proliferation, chemoresistance, and angiogenesis in human cholangiocarcinoma through TEAD transcription factors. Hepatology 2015;62:1497-1510.

28. Paradis V, Dargere D, Bonvoust F, Vidaud M, Segarini P, Bedossa P. Effects and regulation of connective tissue growth factor on hepatic stellate cells. Lab Invest 2002;82:767-774.

29. Rachfal AW, Brigstock DR. Connective tissue growth factor (CTGF/CCN2) in hepatic fibrosis. Hepatol Res 2003;26:1-9.

30. Claperon A, Mergey M, Nguyen Ho-Bouldoires TH, Vignjevic D, Wendum D, Chretien Y, Merabtene $F$, et al. EGF/EGFR axis contributes to the progression of cholangiocarcinoma through the induction of an epithelial-mesenchymal transition. J Hepatol 2014;61:325-332.

31. Socoteanu MP, Mott F, Alpini G, Frankel AE. c-Met targeted therapy of cholangiocarcinoma. World J Gastroenterol 2008;14:2990-2994.

32. Zhang M, Yang H, Wan L, Wang Z, Wang H, Ge C, Liu Y, et al. Single cell transcriptomic architecture and intercellular crosstalk of human intrahepatic cholangiocarcinoma. J Hepatol 2020.

33. Vandewalle C, Van Roy F, Berx G. The role of the ZEB family of transcription factors in development and disease. Cell Mol Life Sci 2009;66:773-787.

This article is protected by copyright. All rights reserved 
34. Chen Z, Zhang N, Chu HY, Yu Y, Zhang ZK, Zhang G, Zhang BT. Connective Tissue Growth Factor: From Molecular Understandings to Drug Discovery. Front Cell Dev Biol 2020;8:593269.

35. Shen YW, Zhou YD, Chen HZ, Luan X, Zhang WD. Targeting CTGF in Cancer: An Emerging Therapeutic Opportunity. Trends Cancer 2021;7:511-524.

36. Gardini A, Corti B, Fiorentino M, Altimari A, Ercolani G, Grazi GL, Pinna AD, et al. Expression of connective tissue growth factor is a prognostic marker for patients with intrahepatic cholangiocarcinoma. Dig Liver Dis 2005;37:269-274.

37. Picozzi V, Alseidi A, Winter J, Pishvaian M, Mody K, Glaspy J, Larson T, et al. Gemcitabine/nab-paclitaxel with pamrevlumab: a novel drug combination and trial design for the treatment of locally advanced pancreatic cancer. ESMO Open 2020;5.

38. de Barrios O, Sanchez-Moral L, Cortes M, Ninfali C, Profitos-Peleja N, Martinez-Campanario MC, Siles L, et al. ZEB1 promotes inflammation and progression towards inflammation-driven carcinoma through repression of the DNA repair glycosylase MPG in epithelial cells. Gut 2019;68:2129-2141.

39. Garcia-Vilas JA, Medina MA. Updates on the hepatocyte growth factor/c-Met axis in hepatocellular carcinoma and its therapeutic implications. World J Gastroenterol 2018;24:3695-3708.

40. Gao R, Brigstock DR. Connective tissue growth factor (CCN2) induces adhesion of rat activated hepatic stellate cells by binding of its C-terminal domain to integrin alpha(v)beta(3) and heparan sulfate proteoglycan. J Biol Chem 2004;279:8848-8855.

41. Gentilini A, Pastore M, Marra F, Raggi C. The Role of Stroma in Cholangiocarcinoma: The Intriguing Interplay between Fibroblastic Component, Immune Cell Subsets and Tumor Epithelium. Int J Mol Sci 2018;19.

42. Sirica AE. The role of cancer-associated myofibroblasts in intrahepatic cholangiocarcinoma. Nat Rev Gastroenterol Hepatol 2011;9:44-54.

43. Ho LJ, Luo SF, Lai JH. Biological effects of interleukin-6: Clinical applications in autoimmune diseases and cancers. Biochem Pharmacol 2015;97:16-26.

44. Katsura A, Tamura Y, Hokari S, Harada M, Morikawa M, Sakurai T, Takahashi K, et al. ZEB1-regulated inflammatory phenotype in breast cancer cells. Mol Oncol 2017;11:12411262.

This article is protected by copyright. All rights reserved 
"Author names in bold designate shared co-first authorship"

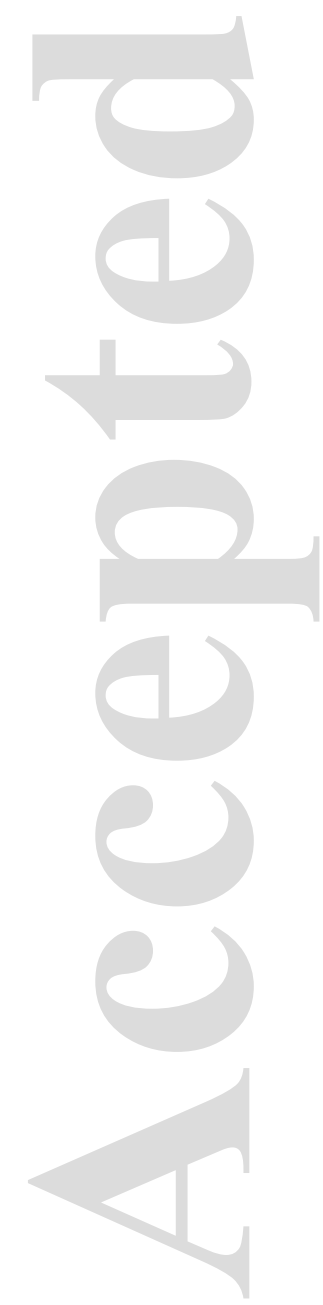

This article is protected by copyright. All rights reserved 


\section{Author contributions:}

Conceptualization and design: JV, LF.

Data generation: CL, MV, AA, EGS, LI, AP, NG, CL, CC, JV.

Data analysis and interpretation: CL, AA, EGS, NG, CL, VP, CC, JV, LF.

Manuscript preparation: CC, NG, JV, LF.

Review and editing: CL, MV, AA, EGS, LI, AP, NG, CL, VP, JMB, CC, CH, JV, LF.

Funding acquisition: CC, JMB, JV, LF.

Acknowledgment: We acknowledge Tatiana Ledent from Housing and experimental animal facility (HEAF), CRSA, Annie Munier and Romain Morichon from the Flow cytometry-imaging platform LUMIC, CRSA, and Claire Calmel from CRSA for help in genotyping CCA cells, Sylvie Fabrega from the "Viral Vector and Gene Transfer Platform, Université Paris Descartes-Sorbonne Paris Cité, SFR Necker, US 24, 75014 Paris, France ». We thank Yves Chrétien for helping with statistical analysis, Miguel Alburquerque for his technical help with immunohistochemistry scanning, and Julie Caramel for providing the plasmid encoding Zeb1 for retroviral overexpression. We thank Kevin Bevant, Katyana Amilca and Michèle Sabbah for their guidance through ChIP experiments and analyses. We thank CERCA Programme / Generalitat de Catalunya for institutional support. 


\section{Figure legends}

Figure 1. ZEB1 expression is related to tumor dedifferentiation in CCA. A. Representative IHC staining of ZEB1 in human non-tumoral liver (left panel), Grade 1 (middle panel) and Grade 3 (right panel) intrahepatic CCA. B-C. Quantification of CCA with ZEB1 positive signal in tumor epithelial cells according to tumor grade $(B)$ and vascular invasion (C). Statistics were performed by applying the Chi square test. D. ZEB1, E-cadherin and vimentin protein expression in a panel of 8 human CCA cell lines, as determined by western blot. E. Correlation between CDH1, ESRP1 and VIM and ZEB1 expression in Rennes and TCGA CCA cohorts, determined using Spearman's correlation analysis.

Figure 2. ZEB1 gain/loss of function in in vitro models derived from CCA cell lines. A. Schematic representation of the generation of gain/loss of function cellular models derived from HuCCT1 (which express negligible levels of ZEB1), SK-ChA-1 cells (which express high levels of ZEB1) and HuCCT1 Resistant (R) cells to erlotinib (which express high levels of ZEB1 as cause of the resistance). B. Representative phase contrast pictures showing the mesenchymal or epithelial morphology. C. Changes in ZEB1 mRNA and protein expression, as determined by RTQPCR and western blot. Values are expressed as means \pm SEM from 5 independent cultures. *, $\mathrm{p}<0.05 ;{ }^{* *}, \mathrm{p}<0.01 ;{ }^{* * *}, \mathrm{p}<0.001,{ }^{* * * *}, \mathrm{p}<0.0001$, as compared with control cells or shControl clone 1. \#\#\#, $\mathrm{p}<0.0001$, as compared with shControl clone 2.

Figure 3. ZEB1 expression promotes the acquisition of an EMT/CSC phenotype in CCA cells. A-C. Changes in CDH1 (E-cadherin), ESRP1 and VIM (vimentin) mRNA and protein expression and localization in HuCCT1 control cells or cells overexpressing Zeb1, as determined by RT-QPCR, western blot and immunofluorescence, respectively. Scale: $50 \mu \mathrm{m}$. D-E. Changes in CD44 and CD24 mRNA and protein expression in the same cells, as determined by RT-QPCR (D) and by flow cytometry (E). Quantification in the right panel shows the \% of cells in the lower right quadrant corresponding to a CD44 $/ \mathrm{CD} 24^{-}$population. F. Cell tracking analyzed by timelapse microscopy during $24 \mathrm{~h}$. Tracks of 8 representative cells for each condition are shown. Bar graphs show quantification of the distance travelled by the cells. G-H. Colony $(G)$ and sphere $(H)$ formation assay after 7 days in culture. Representative pictures of colonies and spheres are shown. Scale: $100 \mu \mathrm{m}$. Values are expressed as means \pm SEM from at least 3 independent cultures. *,$p<0.05 ;{ }^{* *}, p<0.01 ;{ }^{* * *}, p<0.001,{ }^{* * *}, p<0.0001$, as compared with Control cells.

Figure 4. ZEB1 expression promotes tumorigenicity and stroma expansion in CCA xenografts. A. Tumor volume of mice bearing HuCCT1 Control (white) or Zeb1 (black) cells. BD. Representative images of tumors (B), HE (C) and Picro-Sirius Red stainings (D) from HuCCT1 
control and Zeb1 xenograft tumors. Scale: $50 \mu \mathrm{m}$. Quantification of collagen positive area was analyzed with ImageJ. E. mRNA expression of mouse Acta2 (a-SMA), Col1a1 and Col4a1 in xenograft tumors derived from HuCCT1 Control and Zeb1 cells. F-G. mRNA expression of TGFB1, CCN2 and PDGFB in tumor cells (corresponding to human genes) in xenograft tumors derived from HuCCT1 Control and Zeb1 cells (F), or HuCCT1 WT and Resistant (R) cells (G). Values are expressed as means \pm SEM from 5 animals. ${ }^{*}, p<0.05$; ${ }^{* *}, p<0.01$; comparing with control or WT tumors. H-I. Changes in CTGF protein expression in xenograft tumors derived from HuCCT1 Control and Zeb1 cells $(\mathrm{H})$, or HuCCT1 WT and R cells (I), as determined by western blot.

Figure 5. ZEB1 regulates the expression of CCN2/CTGF in CCA. A. CCN2 (CTGF) mRNA and protein expression in gain/loss of function models derived from SK-ChA-1, HuCCT1 and HuCCT1 Resistant $(R)$ cells. Results are expressed as means \pm SEM from 5 independent cultures. B. Correlation between CCN2 and ZEB1 expression in the Rennes and the TCGA CCA cohorts. C. Effect of recombinant CTGF $100 \mathrm{ng} / \mathrm{ml}$ on the viability of hTERT-HSC cells. D. Effect on the viability of hTERT-HSC cells of conditioned media (CM) from HuCCT1 Control (white bar) and CM from HuCCT1-Zeb1 cells in absence (black) or presence of an unspecific IgG (dark grey) or a CTGF neutralizing antibody (light grey). E. Chromatin immunoprecipitation PCR (ChIP-PCR) experiment using primers targeting CCN2 promoter in HuCCT1 Control (white bars) and Zeb1 (black bars) cells. ${ }^{*}, \mathrm{p}<0.05 ;{ }^{* *}, \mathrm{p}<0.01 ;{ }^{* * *}, \mathrm{p}<0.001$, as compared with Control cells, shRNAControl clone 1 or vehicle. \#, p<0.01; \#\#, p<0.001, as compared with shRNA-Control clone 2.

Figure 6. ZEB1 expression in cancer-associated fibroblasts impacts their activation status. A. Representative IHC co-immunostaining of ZEB1 (brown) and $\alpha$-SMA (pink) in human iCCA showing ZEB1 expressing CAF. B. Correlation between ACTA2 ( $\alpha-S M A), C O L 4 A 1, T G F B 1$ and CCN2 (CTGF) with ZEB1 expression in microdissected stroma from 10 iCCA samples. C-D. mRNA expression of Zeb1 in the stromal compartment (corresponding to murine genes) in xenograft tumors derived from HuCCT1 Control and Zeb1 cells (C), or from HuCCT1 WT and Resistant (R) cells (D). E. Changes in mRNA and protein expression of ZEB1, determined by RTQPCR and WB in hTERT-HSC shControl or shZEB1 cells. F. mRNA expression of TGFB1, CCN2 (CTGF), PDGFB, ACTA2 ( $\alpha$-SMA) and COL4A1, in hTERT-HSC shControl or shZEB1 cells. Results are expressed as means \pm SEM from 5 animals or 5 independent cultures. *, $p<0.05$; **, $p<0.01 ;{ }^{* * *}, p<0.001$, as compared with Control tumors, WT tumors or shControl cells.

Figure 7. Depletion of ZEB1 in myofibroblasts reduces CCA progressiveness. A. Tumor volume of mice bearing EGI-1+hTERT-HSC-shControl or EGI-1+hTERT-HSC-shZEB1 cells. Representative images of tumors are shown. B. mRNA expression of Zeb1, Acta2 ( $\alpha-S m a)$, 
Col4a1, Tgfb1, Ccn2 (Ctgf) and Pdgfb in the stromal compartment (corresponding to murine genes). C. Effect of conditioned media (CM) from hTERT-HSC shControl or shZEB1 cells on the viability of EGI-1 and HuCCT1 cells. D. Representative image of western blot analysis of the phosphorylated and total forms of STAT3, AKT and ERK after 30 minutes of exposure to the indicated $\mathrm{CM}$ in EGI-1 and HuCCT1 cells. Results are expressed as means \pm SEM from 5 animals or 5 independent cultures. ${ }^{*}, \mathrm{p}<0.05$; ${ }^{* *}, \mathrm{p}<0.01$; ${ }^{* *}, \mathrm{p}<0.001$, as compared with shControl tumors or vehicle. \#, p<0.01; \#\#, as compared with CM from hTERT-HSC shControl.

Figure 8. ZEB1 expression in tumor cells and CAF impacts their crosstalk. A and C. mRNA expression of HGF (A) and IL6 (C) in HuCCT1 Control and Zeb1, HuCCT1 WT and Resistant (R) CCA cell lines, and hTERT-HSC-shRNA-Control and shRNA-ZEB1 cell lines, and their respective derived CCA xenografts. For each model, gene expression in cultured cells (in vitro), tumor cells (corresponding to human genes) and stromal cells (corresponding to murine genes) of the xenografts (in vivo) is shown. B. mRNA expression of HGF in hTERT-HSC cells exposed to conditioned media (CM) from HuCCT1 Control or Zeb1 cells. D. mRNA expression of ZEB1 and IL6 in hTERT-HSC-shRNA-Control and shRNA-ZEB1 cells after $24 \mathrm{~h}$ after exposure to TGF- $\beta 1$ $10 \mathrm{ng} / \mathrm{ml}$ or the vehicle. E-F. Effect of HGF (F) and IL6 (G) on the viability of CCA and fibroblast cell lines. G. Schematic diagram depicting the major findings of the study. Overexpression of ZEB1 leads to induction of EMT and stemness in tumor cells and to fibroblast activation. Furthermore, ZEB1 at both sides of the tumor-stroma interface, regulates the production of soluble signaling molecules (such as CTGF, HGF and IL6) to boost tumor progression. Values are expressed as means \pm SEM from 5 independent cultures/animals. ${ }^{*}, p<0.05 ;{ }^{* *}, p<0.01 ;{ }^{* *}$, $p<0.001 ;{ }^{* * *}, p<0.0001$, as compared with Control cells, Control tumors or the vehicle.

This article is protected by copyright. All rights reserved 


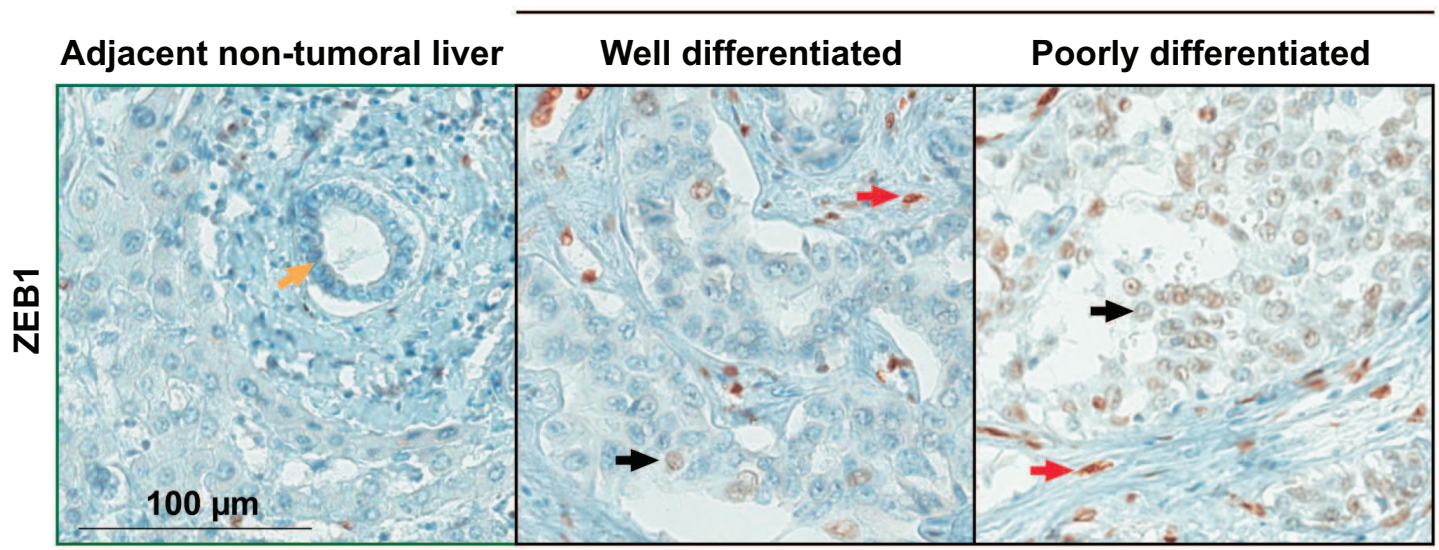

B
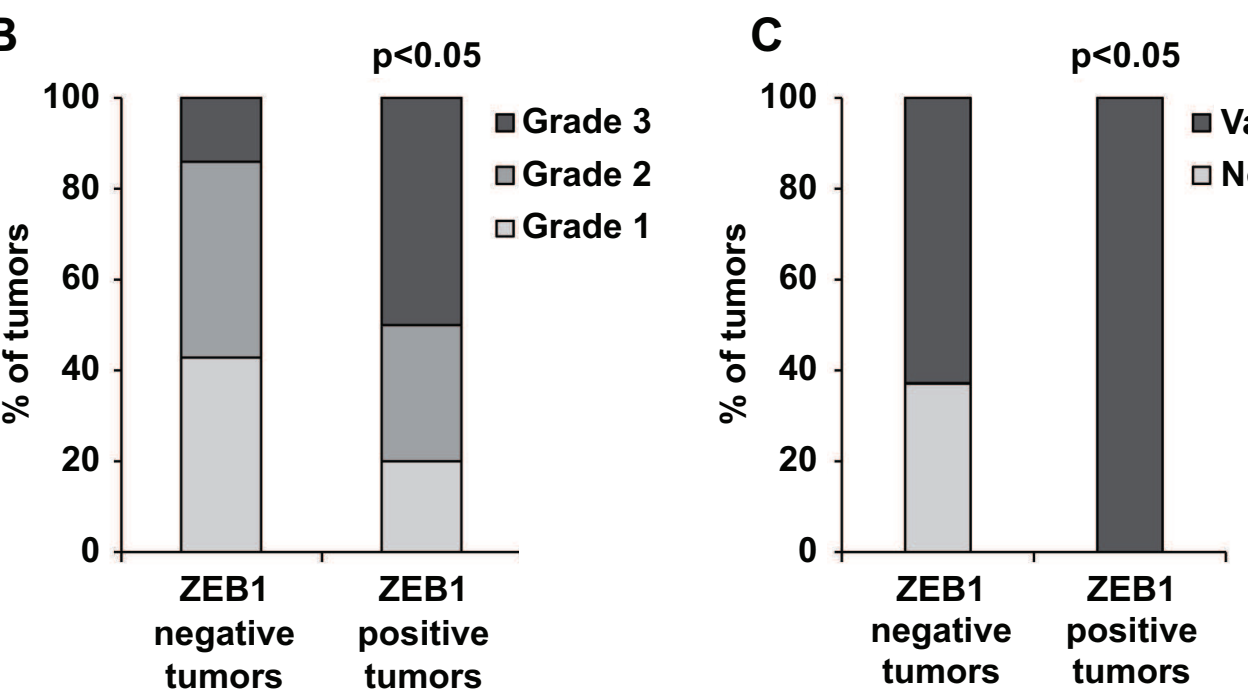

D

E

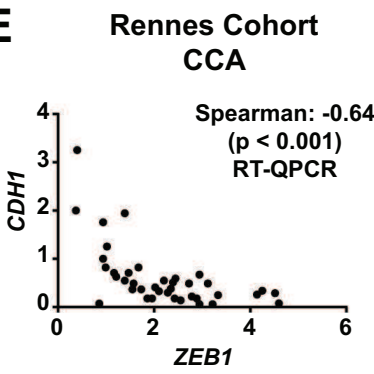

TCGA, PanCancer Atlas
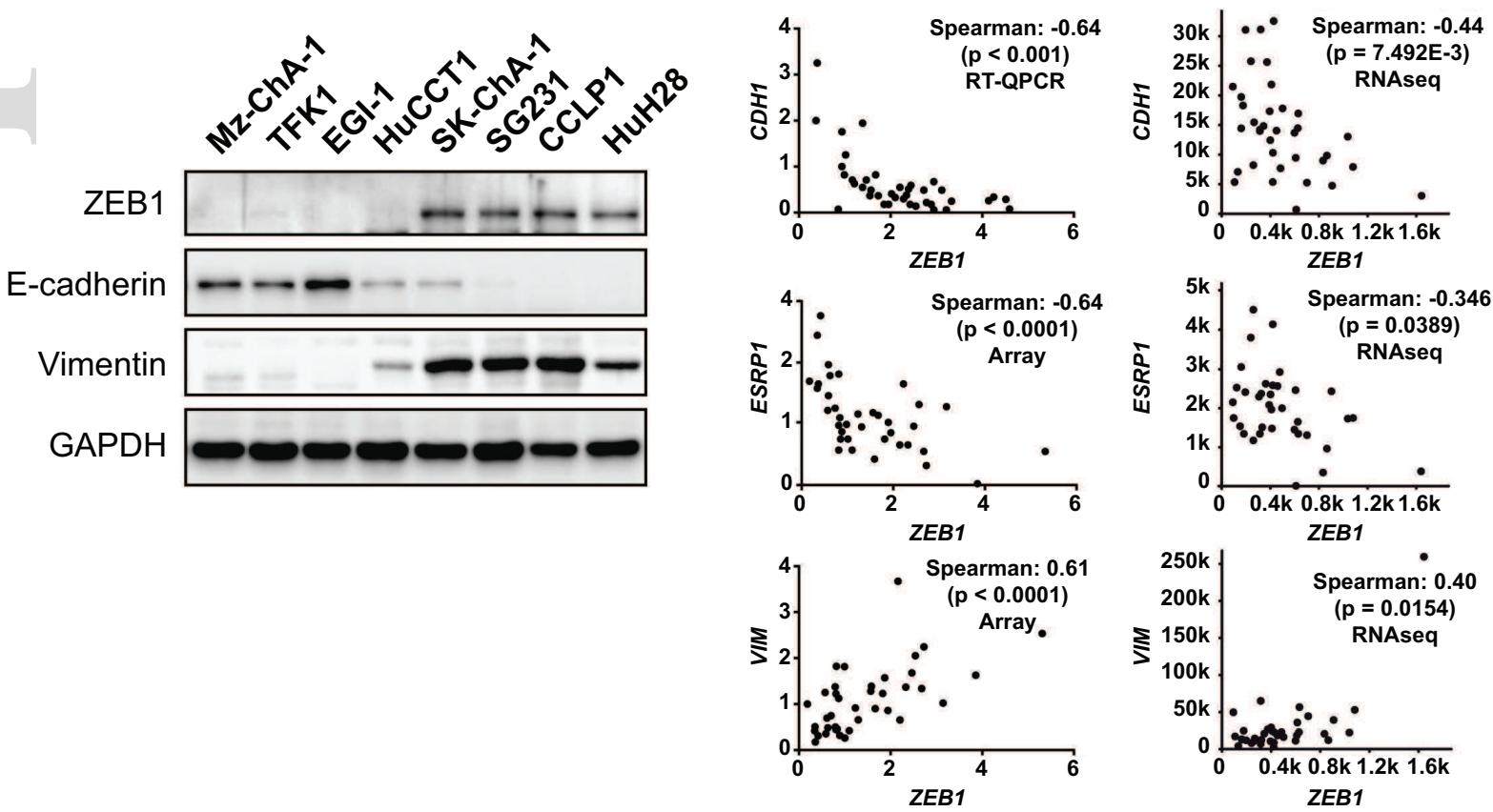

This article is protected by copyrighFighurient reserved 
A

Overexpression model

HuCCT1

(Low ZEB1)
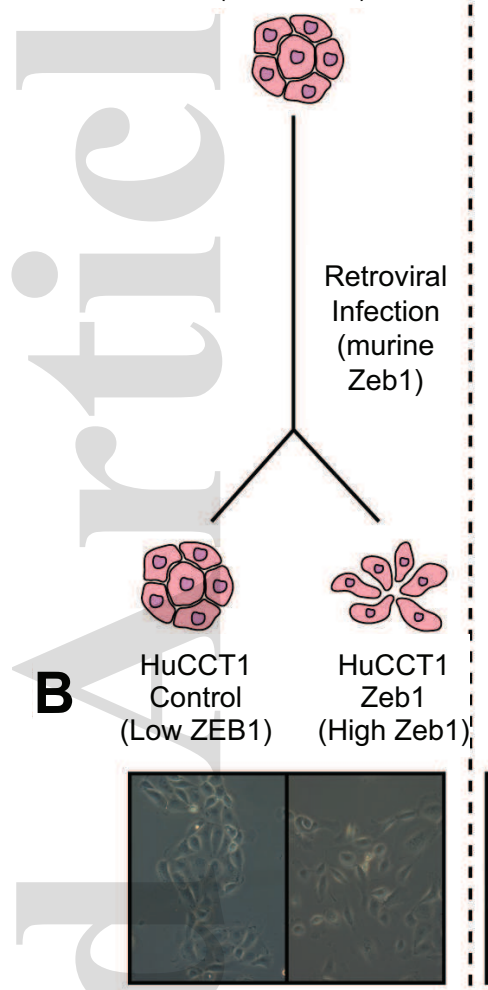

C

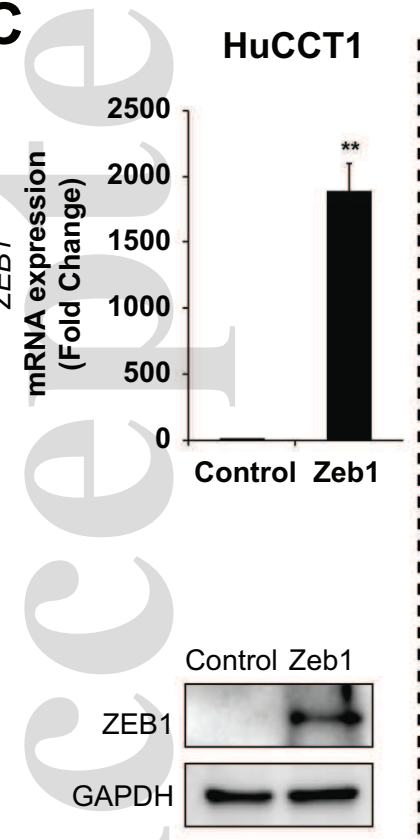

Downregulation model

SK-ChA-1

(High ZEB1)
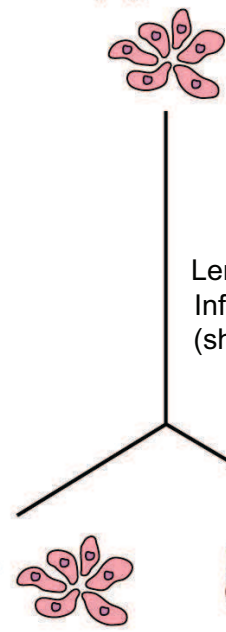

Sk-ChA-1 shControl

Clones $1 \& 2$

(High ZEB1)

Lentiviral

Infection

(shRNA)

\section{Overexpression \& downregulation model}

HuCCT1

(Low ZEB1)
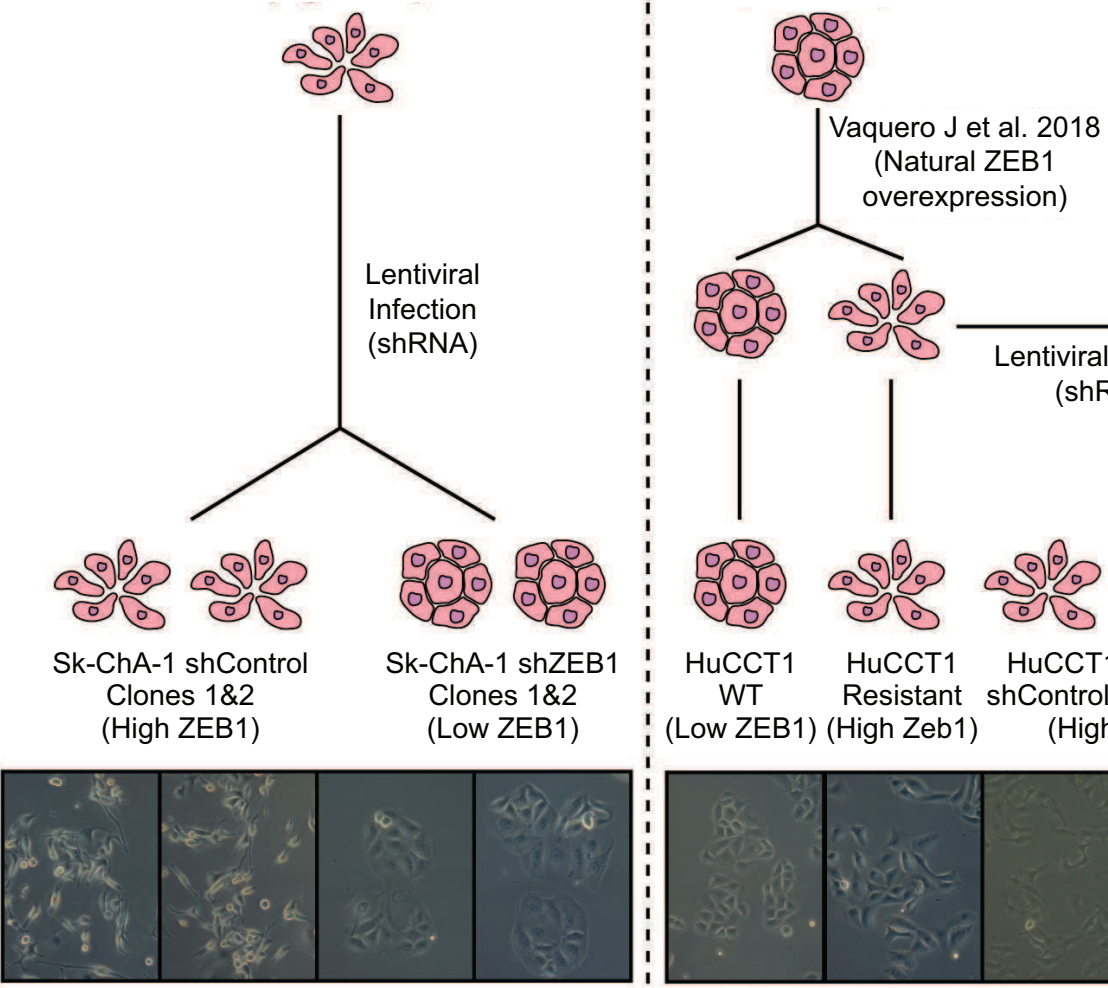

Sk-ChA-1 shZEB1 Clones $1 \& 2$ (Low ZEB1) verexpression)

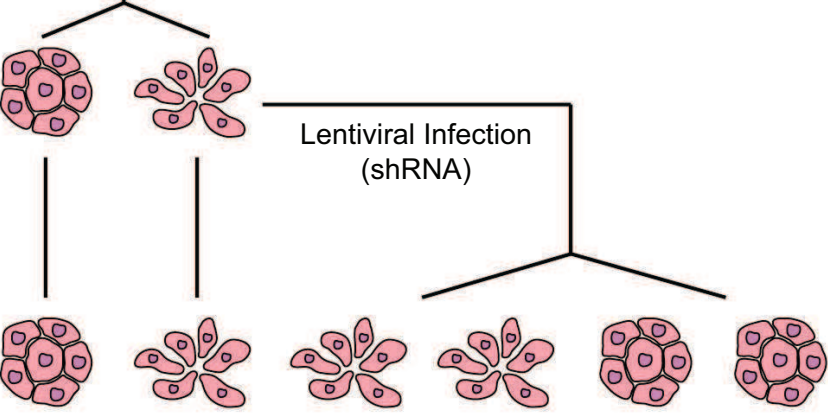

HuCCT1 HuCCT1 HuCCT1 Resistant HuCCT1 Resistant WT Resistant shControl Clones 1\&2 shZEB1 Clones 1\&2 (Low ZEB1) (High Zeb1) (High ZEB1) (Low ZEB1)

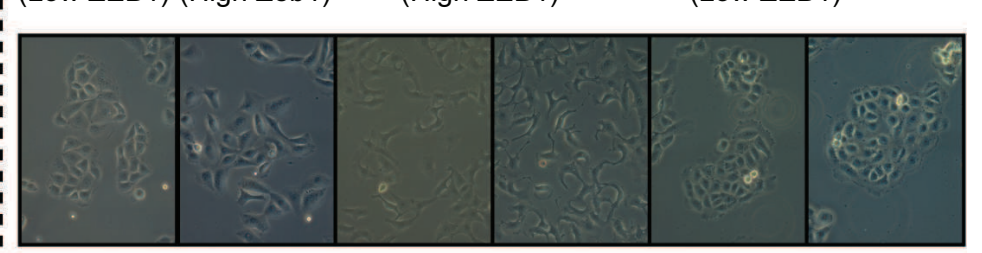

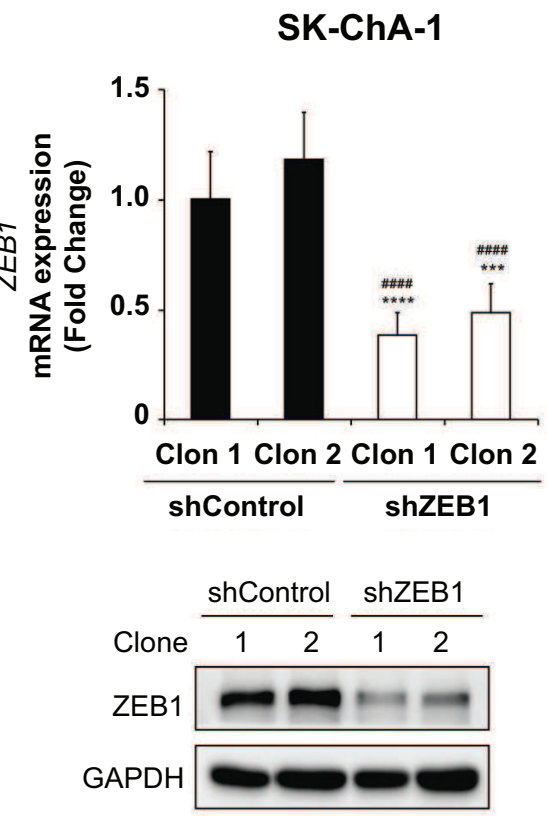

HuCCT1

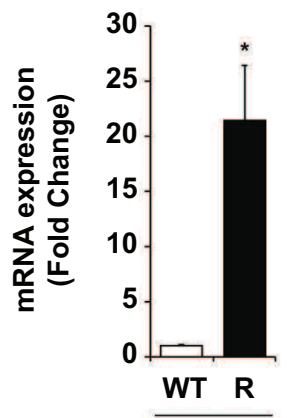

Non Infected

WT R

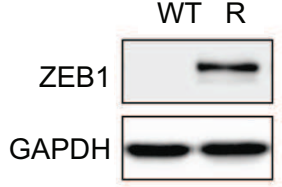

HuCCT1 Resistant
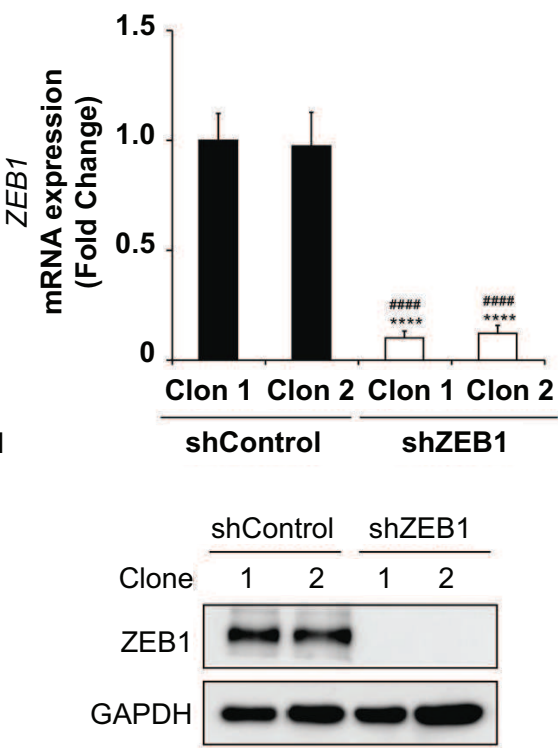

\section{Figure 2}


A
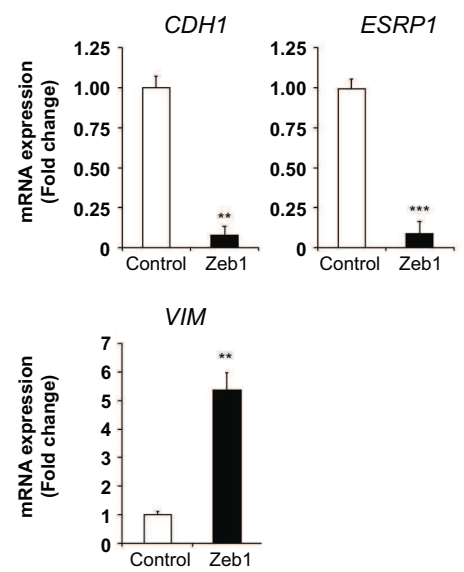

D
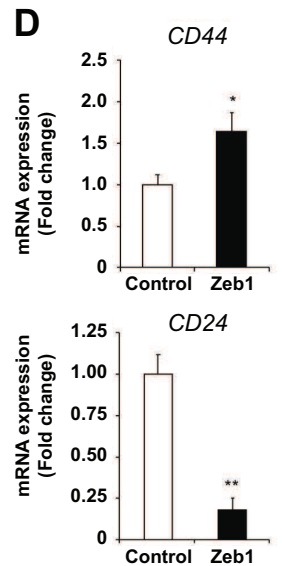

$\mathbf{F}$
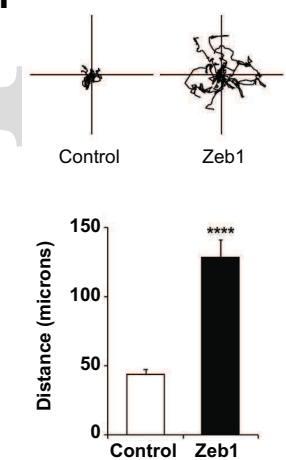

E

G
B
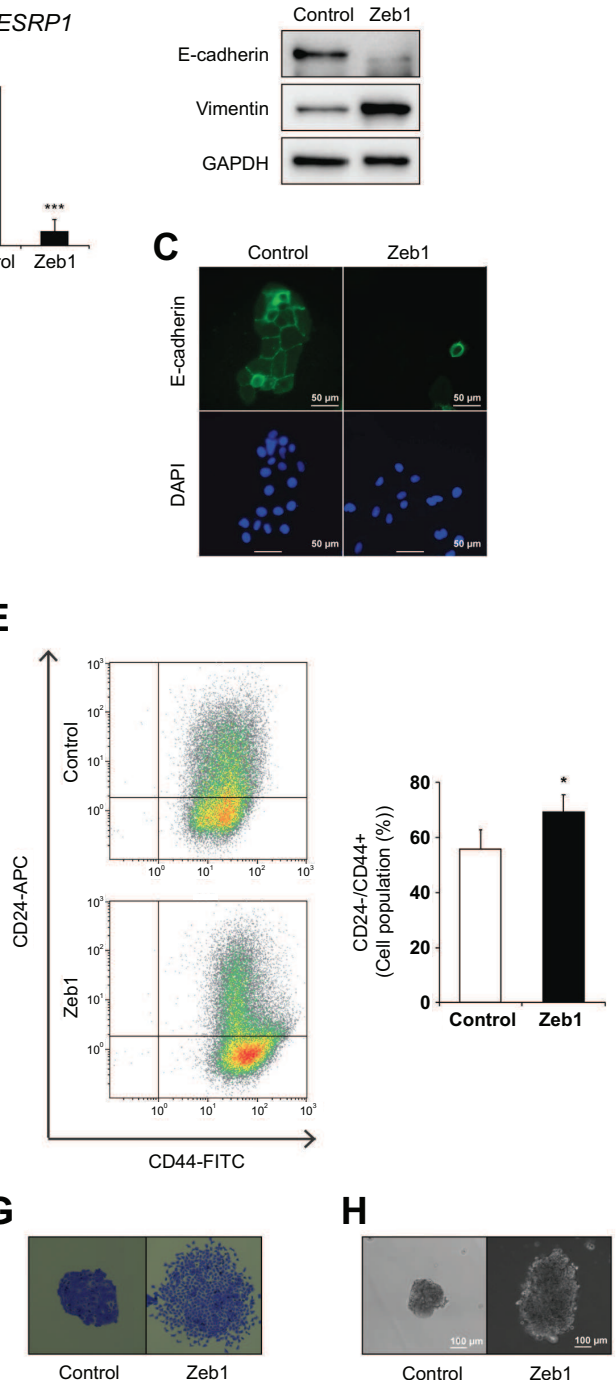

H
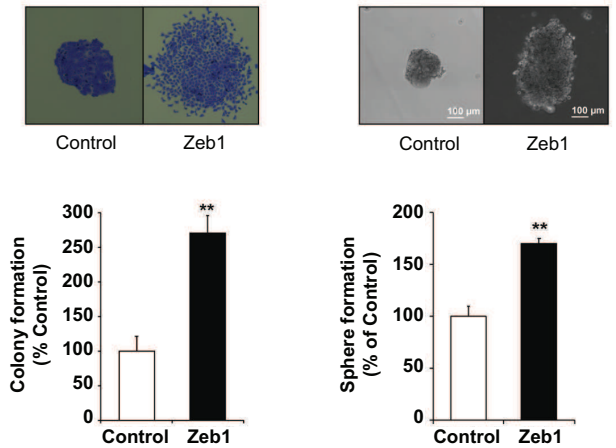

Figure 3 


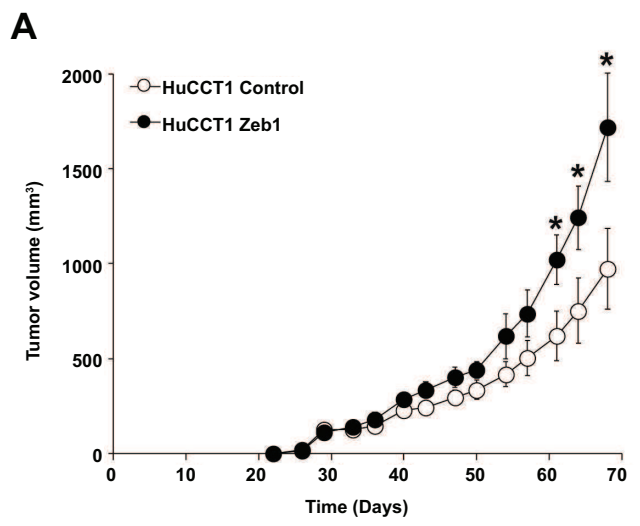

B C
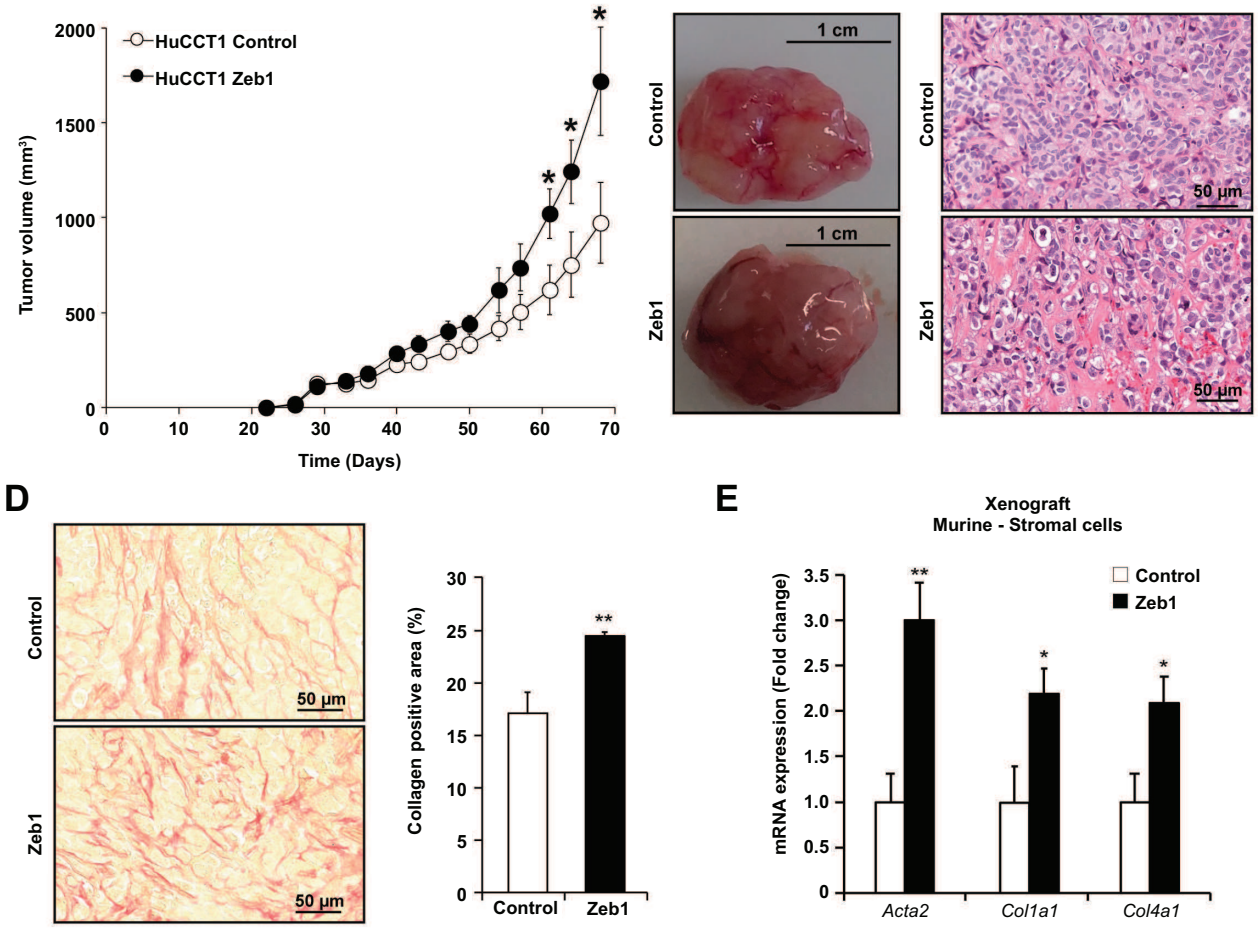

E

Xenograft
Murine - Stromal ce
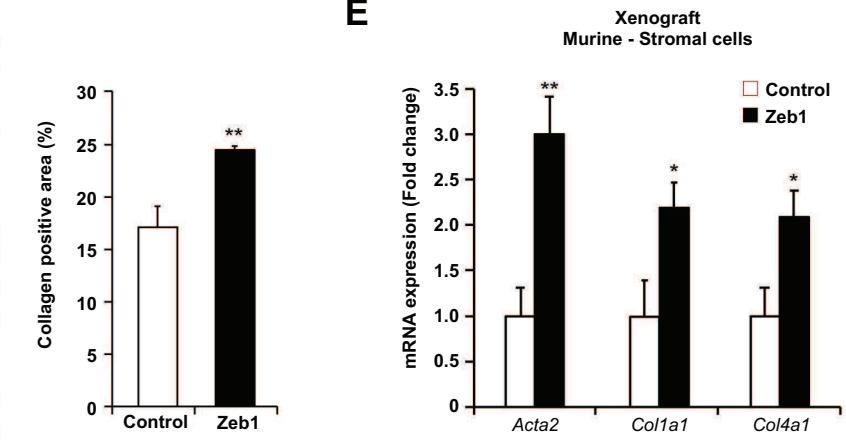

$\mathbf{F}$

Xenograft
Human - Tumor cells

G

Xenograft
Human - Tumor cells
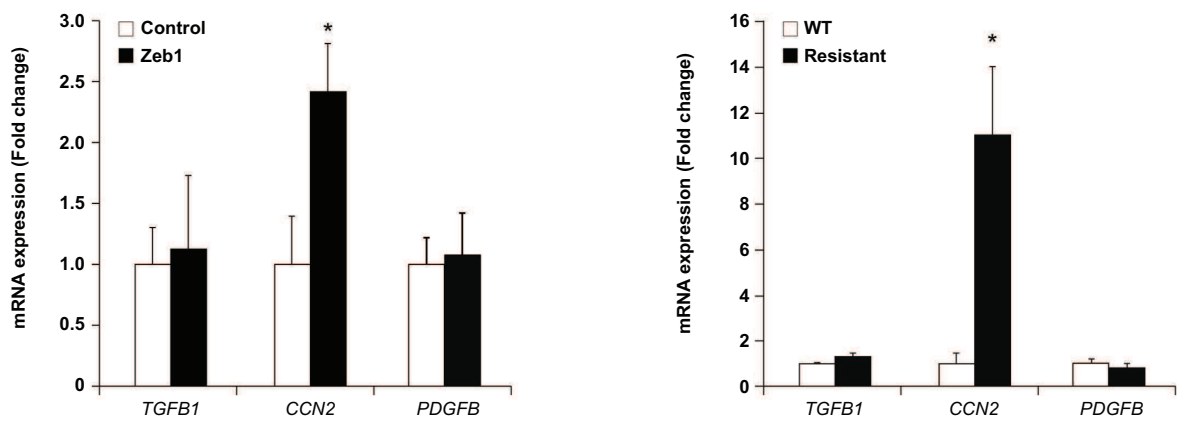

H

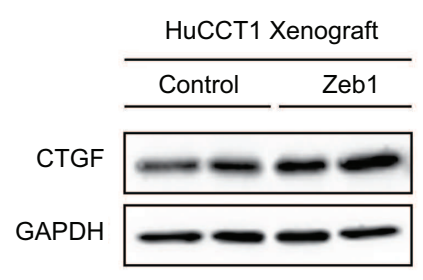

I

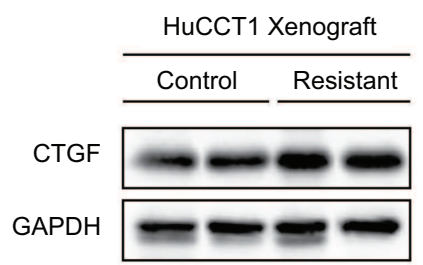

Figure 4

This article is protected by copyright. All rights reserved 
A
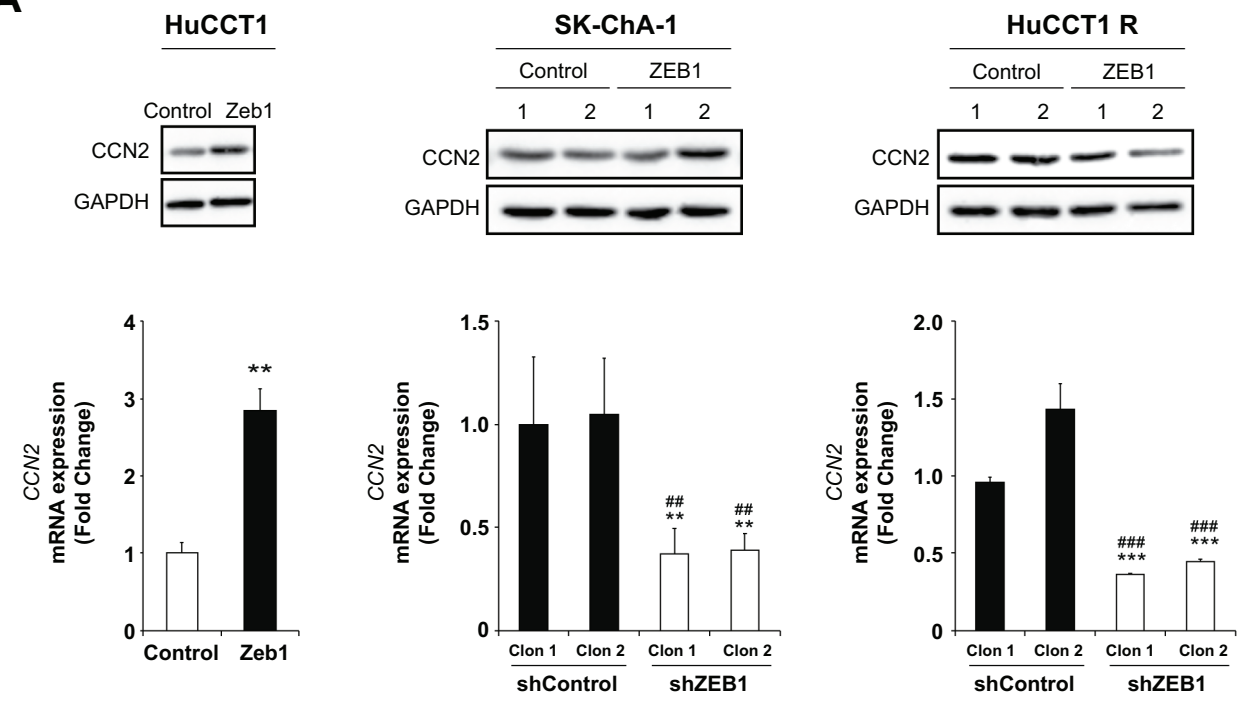

B

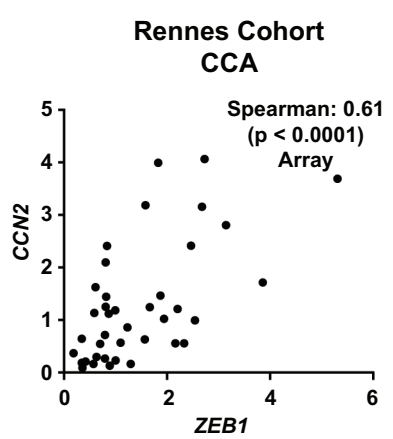

TCGA, PanCancer Atlas CCA
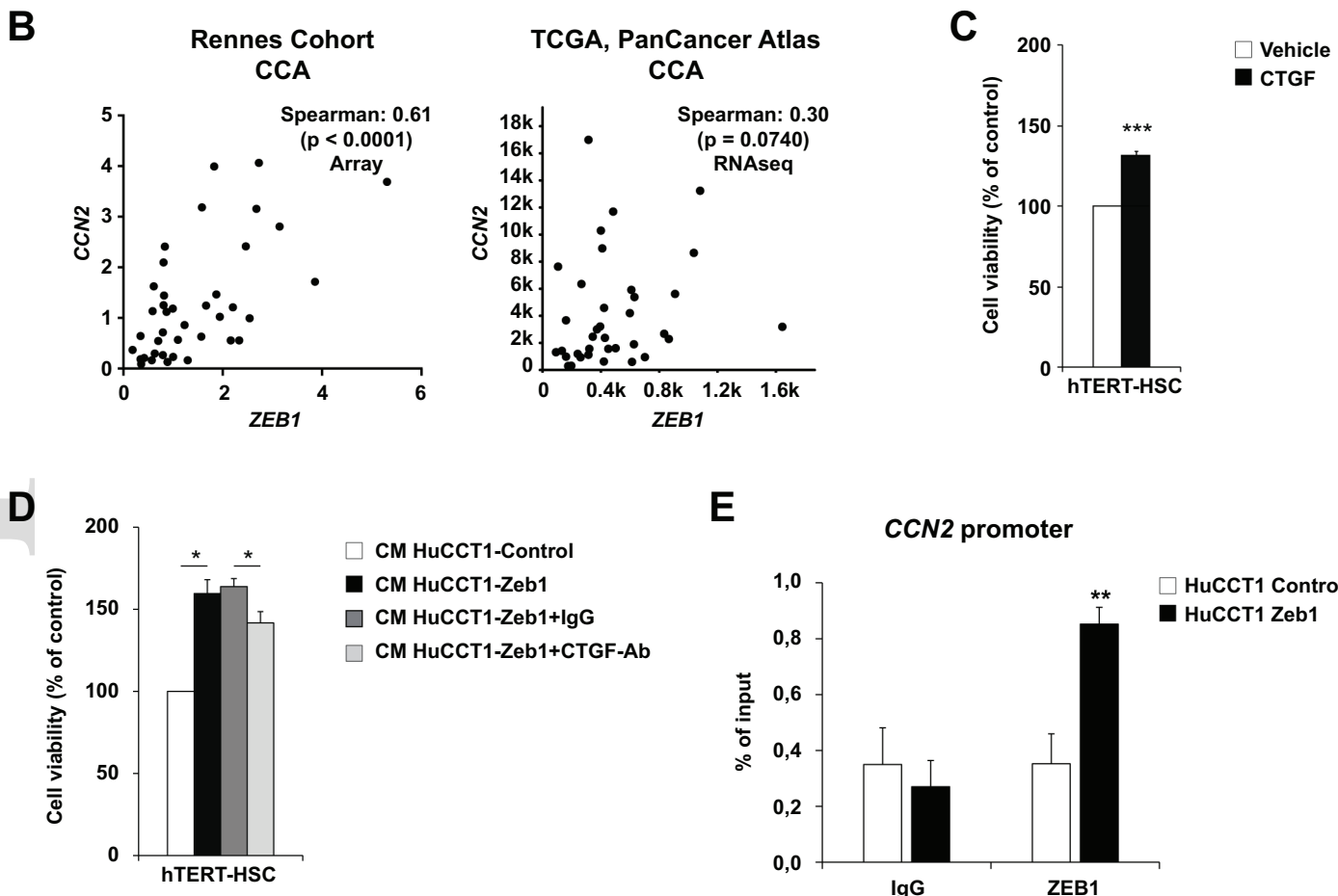

E

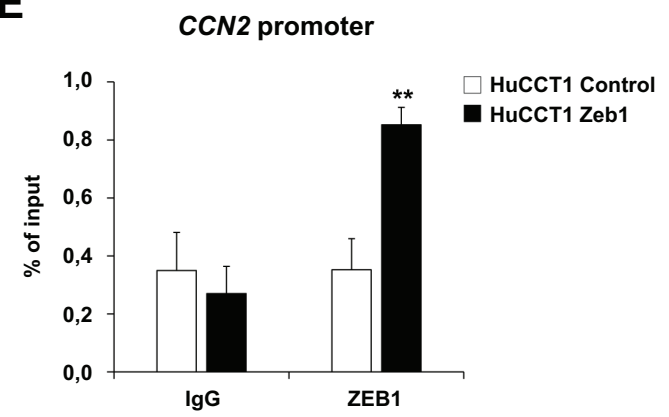

Figure 5 
A
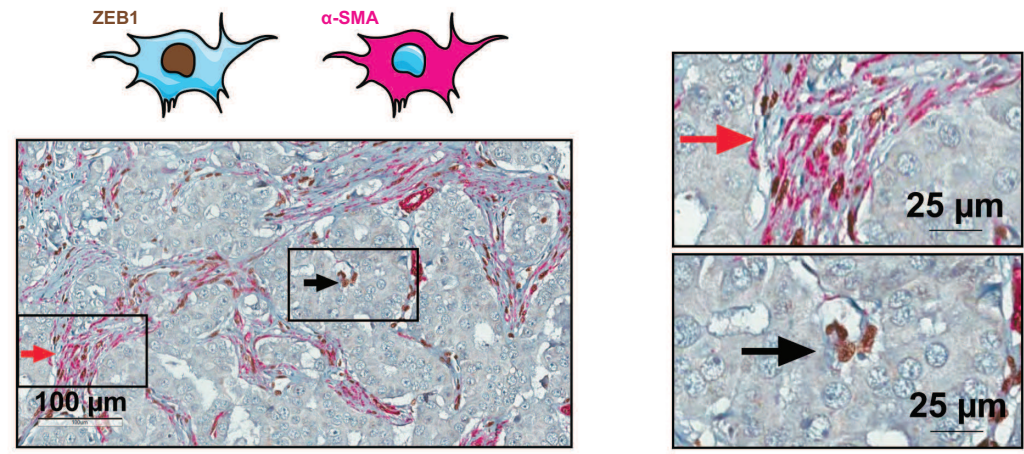

B

Laser microdissected CCA stroma
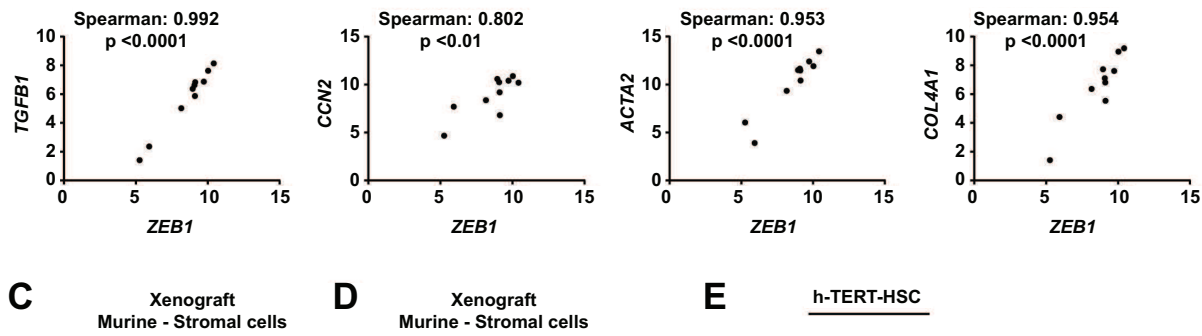

D $\begin{gathered}\text { Xenograft } \\ \text { Murine - Stromal cells }\end{gathered}$

E h-TERT-HSC
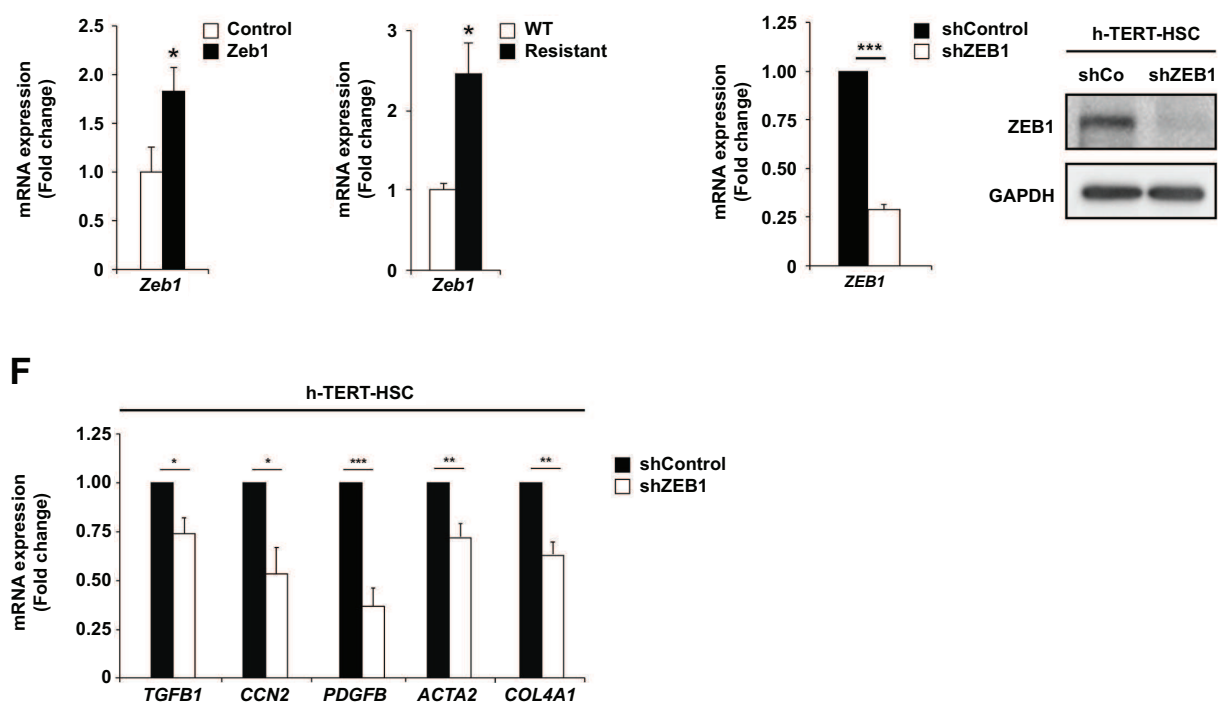

Figure 6

This article is protected by copyright. All rights reserved 
A

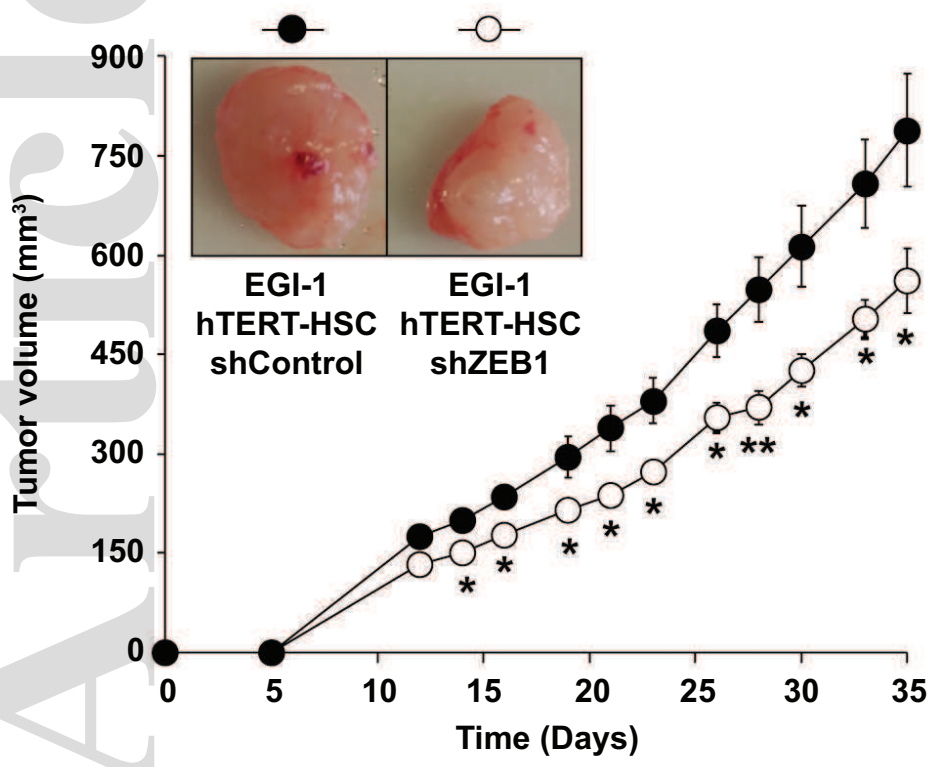

C

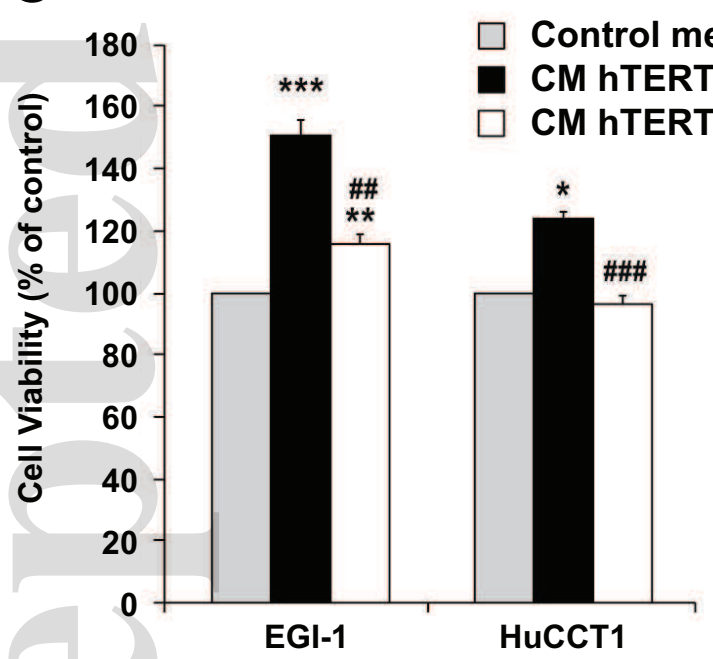

B

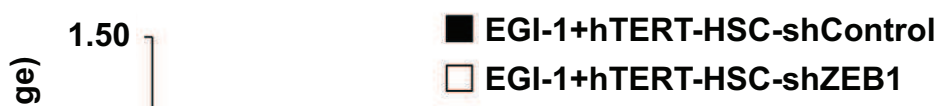

D

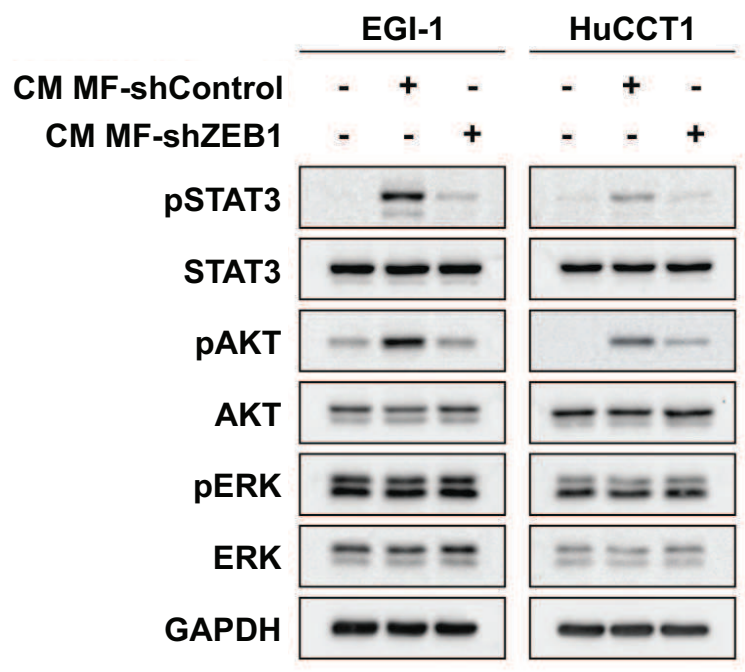

\title{
Figure 7
}

\author{
hep_32069_f7.eps
}


A
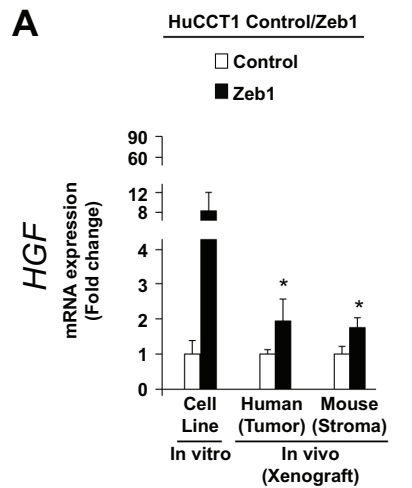

C

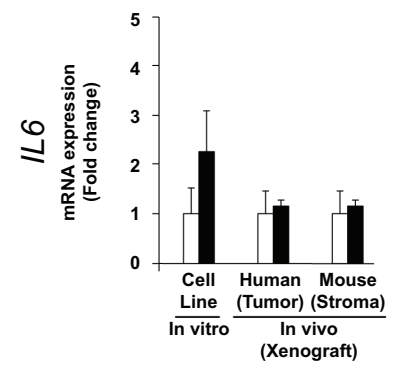

E
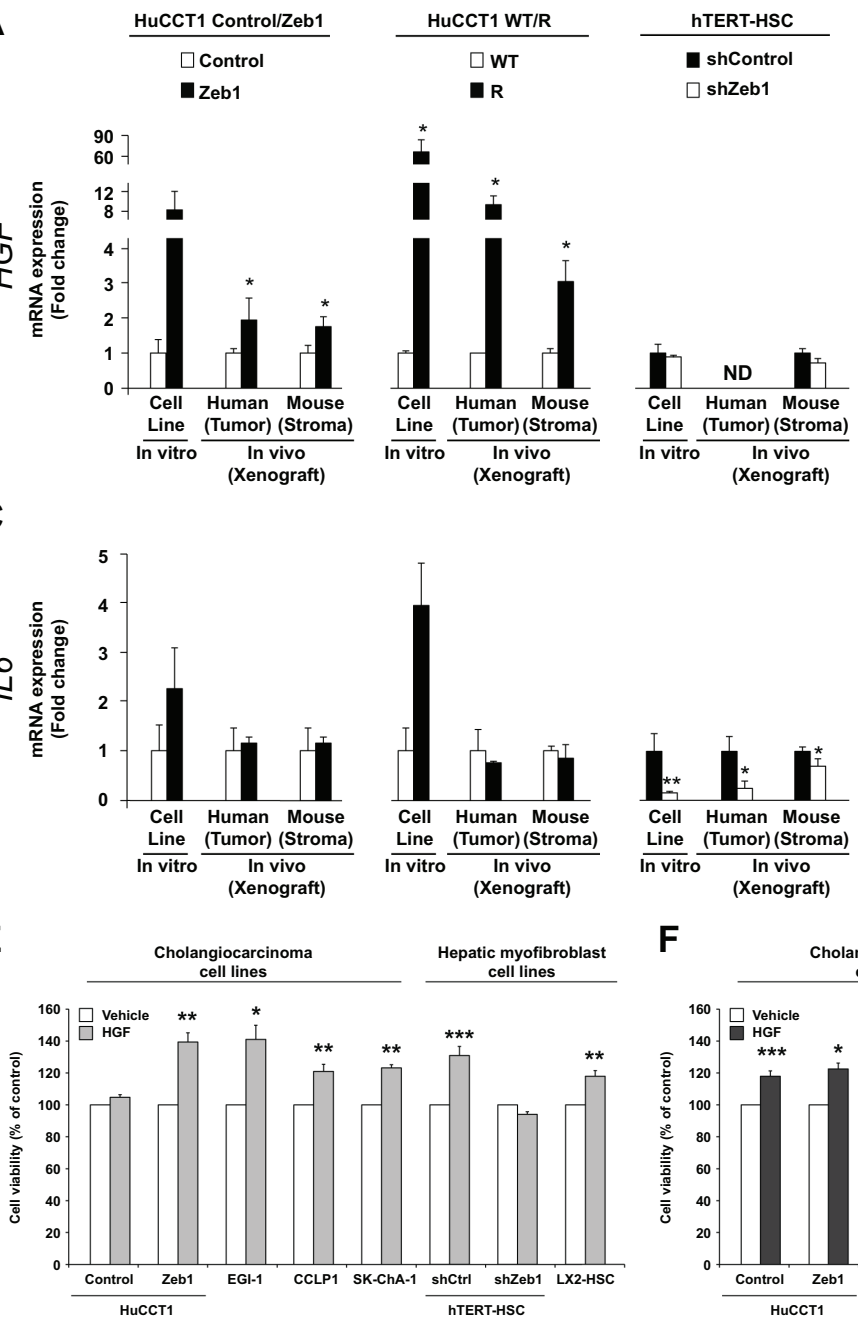

B
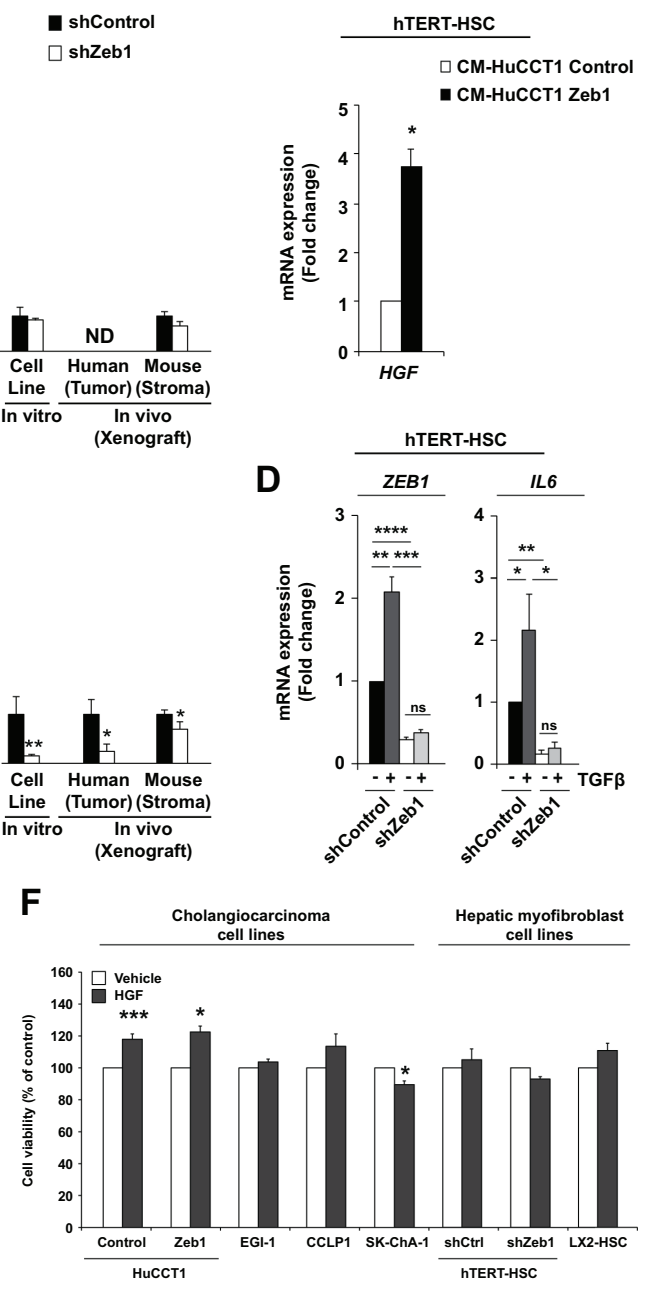
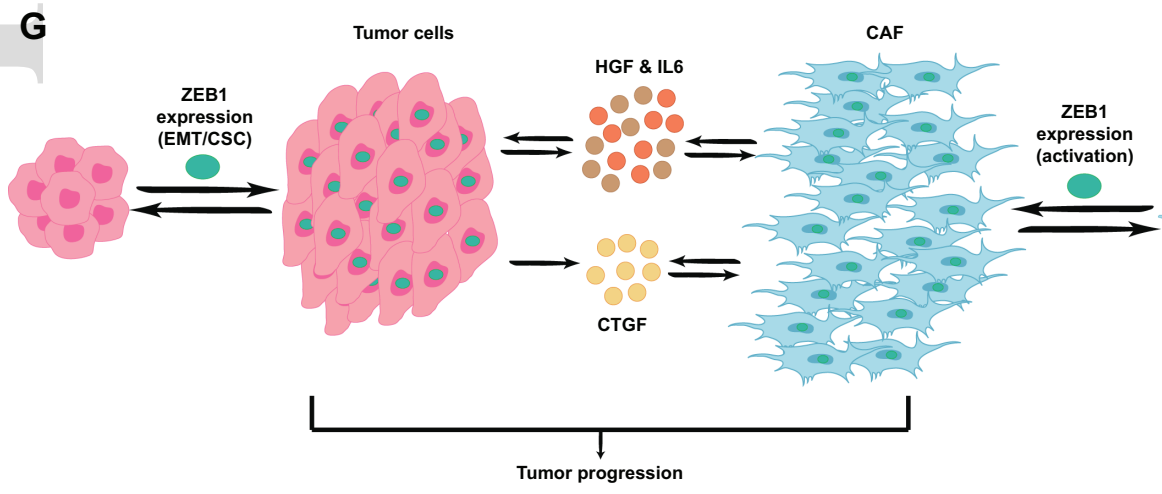

Figure 8 


\section{Supplementary information}

\section{ZEB1 promotes cholangiocarcinoma progression through tumor dedifferentiation and tumor-stroma paracrine signaling}

Cindy Lobe ${ }^{1, *}$, Marie Vallette ${ }^{1, *}$, Ander Arbelaiz ${ }^{1}$, Ester Gonzalez-Sanchez ${ }^{1,2,3}$, Laura Izquierdo $^{3,4}$, Anna Pellat ${ }^{1}$, Nathalie Guedj ${ }^{6}$, Corentin Louis ${ }^{7}$, Valérie Paradis ${ }^{6}$, Jesus M Banales $^{3,4,5}$, Cédric Coulouarn ${ }^{7}$, Chantal Housset ${ }^{1,8}$, Javier Vaquero ${ }^{1,2,3,9, \#, \S}$ \& Laura Fouassier $1, \#, \$$

1-Sorbonne Université, Inserm, Centre de Recherche Saint-Antoine (CRSA), Paris, France. 2-TGF- $\beta$ and Cancer Group, Oncobell Program, Bellvitge Biomedical Research Institute (IDIBELL), Barcelona, Spain.

3-National Biomedical Research Institute on Liver and Gastrointestinal Diseases (CIBERehd), Instituto de Salud Carlos III, Madrid, Spain.

4-Department of Liver and Gastrointestinal Diseases, Biodonostia Health Research Institute Donostia University Hospital, University of the Basque Country (UPV/EHU), San Sebastián, Spain.

5-IKERBASQUE, Basque Foundation for Science, Bilbao, Spain.

6-Service d'Anatomie Pathologique Hôpital Beaujon, F-92110 Clichy, France; INSERM, UMR 1149, Centre de Recherche sur I'Inflammation, F-75018 Paris, France.

7-Inserm UMR 1241, Univ Rennes 1, Rennes, France.

8- Assistance Publique-Hôpitaux de Paris (AP-HP), Saint-Antoine Hospital, Reference center for inflammatory biliary diseases and autoimmune hepatitis, ERN Rare-Liver, Paris, France.

9- LPP, Sorbonne Université, CNRS, Ecole Polytech., Univ. Paris-Sud, Observatoire de Paris, Université Paris-Saclay, PSL Research University, 4 Place Jussieu, 75252 Paris, France.

* Share co-first authorship

\# Share co-senior authorship 


\section{Supplementary Materials and Methods}

\section{Reagents}

CTGF, EGF, HGF, IGF2 and IL6 were purchased from PreproTech.

\section{Cell culture and treatment}

HuCCT1 cells, derived from intrahepatic CCA, were kindly provided by Dr. G. Gores (Mayo Clinic, MN). SG231, CCLP1 and HuH28 cells, derived from intrahepatic CCA were kindly provided by Dr. Cédric Coulouarn (NuMeCan, Rennes, France). EGI-1 and TFK1 cells, derived from extrahepatic CCA, were obtained from the German Collection of Microorganisms and Cell Cultures (DSMZ, Germany). SK-ChA-1 and Mz-ChA-1 cells, derived from extrahepatic CCA, were obtained from Dr. A. Knuth (Zurich University, Switzerland). HuCCT1, CCLP1, EGI-1, Mz-ChA-1 and SK-ChA-1 cells were cultured in DMEM supplemented with $1 \mathrm{~g} / \mathrm{L}$ glucose, 10 mmol/L HEPES. TFK-1 cells were cultured in RPMI supplemented with $\mathrm{HCO}_{3}{ }^{-} 0.75 \%$. SG231 cells were cultured in MEM alpha supplemented with $10 \mathrm{mmol} / \mathrm{L}$ HEPES. HuH28 were cultured in MEM. All culture medias were supplemented with glutamax, $10 \%$ fetal bovine serum (FBS), antibiotics (100 Ul/mL penicillin and $100 \mathrm{mg} / \mathrm{mL}$ streptomycin), and antimycotic $(0.25 \mathrm{mg} / \mathrm{mL}$ amphotericin B; Invitrogen). The human hepatic stellate cell (HSC) lines hTERT-HSC and LX2HSC, were kindly provided by Dr. L. Aoudjehane (ICAN, Paris, France). hTERT-HSC and LX2HSC were cultured in DMEM supplemented with $4.5 \mathrm{~g} / \mathrm{L}$ glucose, antibiotics $(100 \mathrm{Ul} / \mathrm{mL}$ penicillin and $100 \mu \mathrm{g} / \mathrm{mL}$ streptomycin), antimycotic $(0.25 \mathrm{mg} / \mathrm{mL}$ amphotericin $\mathrm{B})$, and $10 \%$ FBS and $2 \%$ FBS, respectively. Cell lines were routinely screened for the presence of mycoplasma and authenticated for polymorphic markers to prevent cross-contamination.

\section{CCN2 RNA silencing}

CCN2 expression was silenced by using a pool of four small interfering RNAs (siRNA) (OnTargetplus SMARTpool, Dharmacon). Control siRNA was a pool from Dharmacon (siGENOME nontargeting siRNA Pool 2). Transient transfections were performed with $100 \mathrm{nM}$ siRNA using DharmaFECT 4 (Thermo Scientific). Cells were plated in 6-well plates and after $24 \mathrm{~h}$ they were 
transfected with the corresponding siRNA. $24 \mathrm{~h}$ after transfection cell culture media was changed to remove transfection complexes. Then, the appropriate protocol for each experiment was followed.

\section{Conditioned media}

HuCCT1-Control, HuCCT1-Zeb1, hTERT-HSC-shControl and hTERT-HSC-shZEB1 cells were grown to confluence, washed twice with phosphate-buffered saline (PBS), and 0\% FBS DMEM (supplemented as above) was added and collected after $48 \mathrm{~h}$. Conditioned media were centrifuged to remove cell debris and stored at $-80^{\circ} \mathrm{C}$ until use. $0 \%$ FBS DMEM was used as control.

\section{Gene expression profiling}

Gene expression datasets established from whole(1) or laser microdissected(2) CCA tumors were previously described. Clinical and pathological features of a cohort of 39 patients with iCCA (thereafter referred to as the Rennes cohort) were previously reported.(1). Freshly frozen tumor samples were obtained through the French liver cancer biobanks network - INCa (BB0033-00085). Of note, because the probe designed for CDH1 (A_23_P206359) was unreliable providing poorly reproducible data, we performed a RT-qPCR analysis using validated pairs of primers for $C D H 1, Z E B 1$ and TBP (housekeeping gene) (Supplementary Table 1) to evaluate the correlation between these genes in the Rennes cohort.

\section{Comparison of mRNA expression from public databases}

The mRNA expression levels of ZEB1 and selected markers (CDH1, VIM, CCN2, ESRP1, IGF2, EGF, HGF and IL6) in human cancer cell lines derived from the Broad-Novartis Cancer Cell Line Encyclopedia (CCLE) and in tumors (breast invasive carcinoma, hepatocellular carcinoma, head and neck squamous cell carcinoma, and, colorectal, lung, pancreatic and prostate adenocarcinomas) from the repository of The Cancer Genome Atlas (TCGA) were analyzed through the cBioPortal database (https://www.cbioportal.org/) and represented as Spearman correlation coefficients $(r)$ and P-values to measure the significance.(3-5) 


\section{RNA and reverse transcription-PCR}

Total RNA extraction and RT-qPCR were performed as previously described.(6) Primer sequences are provided in Supplementary Tables 1, 2 and 3. Gene expression was normalized to GAPDH mRNA content for human genes and to Hprt mRNA content for mouse genes and was expressed relatively to the control condition of each experiment. The relative expression of each target gene was determined from replicate samples using the formula $2^{-\Delta \Delta C t}$.

\section{Chromatin immunoprecipitation PCR (ChIP-PCR) assays}

ChIP assays were performed using the Chromatin Immunoprecipitation Assay Kit from Milipore (\#17-295). $4 \times 10^{6}$ cells were plated in a T75 for $24 \mathrm{~h}$ and sonicated with Bioruptor sonicator (Diagenode) for 20 cycles of 30 seconds sonication and 30 seconds cooling. ChIP was done with $2 \mu \mathrm{g}$ of ZEB1 Antibody from NovusBiologicals (NBP1-05987). Internal control has been done with a normal rabbit IgG (Cell Signaling Technologies) (CST2729). ChIP DNA product and input DNA, extracted by phenol chloroform method, were suspended in $20 \mu \mathrm{L}$ and Input were diluted 1 to 100 , to perform the qPCR $(2 \mu \mathrm{L}$ of sample). Primer sequences are provided in Supplementary Table 4.

\section{Immunoblot analysis}

For obtaining whole-cell lysates for immunoblotting, cell cultures were lysed in RIPA buffer (Sigma) supplemented with $1 \mathrm{mmol} / \mathrm{L}$ orthovanadate and a cocktail of protease inhibitors. Proteins were quantified using a BCA kit (Pierce). Western blot analysis was performed as previously described.(6) Primary antibodies are provided in Supplementary Table 5.

\section{Immunofluorescence}

Immunofluorescence assays were performed as previously described.(6) Primary antibodies are provided in Supplementary Table 5. Cells were observed with an Olympus Bx 61 microscope (Olympus). 


\section{Viability assays}

Cells were plated in 96-well plates. Twenty-four hours later, the medium was replaced by fresh culture medium in the absence (vehicle) or presence of the corresponding growth factors or conditioned media (CM) prepared from HuCCT1 or hTERT-HSC cells. For neutralization experiments unspecific IgG or a neutralizing antibody against CTGF (10 $\mu \mathrm{g} / \mathrm{ml})$ (PreproTech 500-P252) were added to the CM of HuCCT1-Zeb1 cells. Cells were then incubated for $72 \mathrm{~h}$ before determining the viability by the crystal violet method. Absorbance was quantified with a spectrophotometer (Tecan) at $595 \mathrm{~nm}$.

\section{Migration assay}

Cell motility was evaluated by time-lapse microscopy. Cells were plated in 24-well plates (TPP) and cultured for $24 \mathrm{~h}$. Then, medium was replaced for fresh culture medium and images were collected every 20 min for $24 \mathrm{~h}$ with an Olympus Bx 61 microscope (Olympus). During the entire experiment, cells were kept at $37^{\circ} \mathrm{C}$ under $5 \% \mathrm{CO}_{2} .8$ cells were randomly selected in the first frame of the videos and then, their movement was followed in all the frames from 0 to $24 \mathrm{~h}$. Cell trajectories were traced using the center of the nucleus as a reference point and were used to calculate the mean speed of the cells with ImageJ software.

\section{Flow cytometry analysis}

For the determination of the CD44/CD24 phenotype, cells were washed with PBS, detached with trypsin incubation, and resuspended in PBS supplemented with 0.5\% BSA. Combinations of fluorochrome-conjugated monoclonal antibodies against human CD44 (FITC) and CD24 allophycocyanin (APC) were obtained from Beckman Coulter. Specific antibodies or the respective isotype controls were added to the cell suspension, as recommended by the manufacturer, and incubated at $4^{\circ} \mathrm{C}$ for 20 minutes. Cells were washed with PBS containing $0.5 \%$ BSA, centrifuged, and resuspended in PBS with $2 \%$ paraformaldehyde. The labelled cells were analyzed with a Gallios flow cytometer (Beckman-Coulter). 


\section{Colony formation assay}

Cells were plated in 6-well plates at a density of 500 cells/well and then they were cultured for 7 days. Colonies were fixed with $4 \%$ paraformaldehyde, stained with $1 \%$ crystal violet and counted. Only colonies with more than 50 cells were counted.

\section{Sphere formation assay}

Cells were plated in 6-well ultralow attachment plates (Greiner Bio One) in serum-free DMEM/F12 medium, supplemented with $100 \mathrm{mg} / \mathrm{mL}$ gentamycin (Sigma-Aldrich), B27 (Life Technologies), $20 \mathrm{ng} / \mathrm{mL}$ human epidermal growth factor (EGF, Life Technologies), $20 \mathrm{ng} / \mathrm{mL}$ human basic fibroblast growth factor (bFGF, Life Technologies), and 1\% antibiotic-antimycotic solution (Life Technologies) at a density of $4 \times 10^{4}$ cells/well. Fresh sphere medium was added to plates after 4 days. After 7 days, spheres were counted on EVOS FL Cell Imaging System (Thermo Fisher Scientific).

\section{Xenograft tumor model}

Animal experiments were performed in accordance with the French Animal Research Committee guidelines and all procedures were approved by a local ethic committee (APAFIS\#14678-2018041510038914). In a first set of experiments, HuCCT1-Control and HuCCT1-Zeb1 cells $\left(5 \times 10^{6}\right)$ suspended in $60 \mathrm{~mL}$ of PBS were mixed with $60 \mathrm{~mL}$ of Matrigel growth factor reduced (Corning) and implanted subcutaneously into the flank of 5-week-old female NMRI-nu (nu/nu) mice (Envigo). In a second set of experiments EGI-1+hTERT-HSCshControl or EGI-1+hTERT-HSC-shZEB1 $\left(2 \times 10^{6}\right.$ in a ratio 1:1) were injected into the animals following the same protocol. Mice were housed under standard conditions in individually ventilated cages enriched with a nesting material and kept at $22^{\circ} \mathrm{C}$ on a $12-\mathrm{h}$ light/ $12-\mathrm{h}$ dark cycle with ad libitum access to food and tap water. Tumor growth was followed with a caliper, and tumor volume $(\mathrm{V})$ was calculated as follows: xenograft volume $=x y^{2} / 2$, where $x$ is the longest and $\mathrm{y}$, the shortest of two perpendicular diameters. During the experiments, animals showed no sign of toxicity, such as body weight loss (>15\%), decreased food intake, or 
diarrhea. The day of the sacrifice, a slice of the tumor was fixed in 10\% formalin and embedded in paraffin, and the rest was cut in pieces and frozen in liquid nitrogen.

\section{CCA specimens}

We retrospectively retrieved from the files of surgical and pathology department, cases of patients who had undergone liver resection for intrahepatic CCA between 2002 and 2014 at Beaujon Hospital, Clichy, France. All clinicopathological and follow up data of patients were collected and registered in data base (Supplementary Table 6). Informed consent was obtained in all cases.

\section{Immunohistochemical analysis on Tissue MicroArray (TMA)}

Paraffin embedded tissue blocks were used for tissue microarray construction. The slides were reviewed to identify and mark representative areas of viable tumor tissue. Taking tumor heterogeneity into account, three tissue cores of $1 \mathrm{~mm}$ each were punched from selected from selected tumor areas of any donor tissue block and brought into a recipient paraffin block. We used a tissue arraying instrument (MTA-1, Beecher Instrument, Inc., Sun Prairie, WI, USA). A total of 5 TMA were built.

Immunohistochemistry against ZEB1 and smooth muscle actin ( $\alpha$-SMA) (Supplementary Table 5) was realized. Immunohistochemical study was performed on TMA using an automated immunohistochemical stainer according to the manufacturer's guidelines (streptavidinperoxidase protocol; Benchmark, Ventana, Tucson, AZ). All sections were evaluated by pathologists (NG and VP) using a semi-quantitative analysis. ZEB1 was considered positive when nuclear staining in tumor cells was observed whatever the intensity. a-SMA was considered positive when CAF were immunostained.

We also performed a co-immunostaining between ZEB1 and a-SMA according the ultraview double immunostained protocol (optiview DAB/ultraview RED). 


\section{Statistical analysis}

Results were analyzed using the GraphPad Prism 5.0 statistical software. Data are shown as means \pm standard error of the mean (SEM). For comparisons between two groups, parametric Student $\mathrm{t}$ test or nonparametric Mann-Whitney test were used. For comparisons between more than two groups, parametric one-way ANOVA test followed by a posteriori Bonferroni test was used. Histopathological parameters were statistically analyzed Chi-square test. 


\section{Supplementary References.}

1. Angenard G, Merdrignac A, Louis C, Edeline J, Coulouarn C. Expression of long noncoding RNA ANRIL predicts a poor prognosis in intrahepatic cholangiocarcinoma. Dig Liver Dis 2019;51:1337-1343.

2. Sulpice L, Rayar M, Desille M, Turlin B, Fautrel A, Boucher E, Llamas-Gutierrez F, et al. Molecular profiling of stroma identifies osteopontin as an independent predictor of poor prognosis in intrahepatic cholangiocarcinoma. Hepatology 2013;58:1992-2000.

3. Cerami E, Gao J, Dogrusoz U, Gross BE, Sumer SO, Aksoy BA, Jacobsen A, et al. The cBio cancer genomics portal: an open platform for exploring multidimensional cancer genomics data. Cancer Discov 2012;2:401-404.

4. Gao J, Aksoy BA, Dogrusoz U, Dresdner G, Gross B, Sumer SO, Sun Y, et al. Integrative analysis of complex cancer genomics and clinical profiles using the cBioPortal. Sci Signal 2013;6:pl1.

5. Ghandi M, Huang FW, Jane-Valbuena J, Kryukov GV, Lo CC, McDonald ER, 3rd, Barretina J, et al. Next-generation characterization of the Cancer Cell Line Encyclopedia. Nature 2019;569:503-508.

6. Vaquero J, Lobe C, Tahraoui S, Claperon A, Mergey M, Merabtene F, Wendum D, et al. The IGF2/IR/IGF1R Pathway in Tumor Cells and Myofibroblasts Mediates Resistance to EGFR Inhibition in Cholangiocarcinoma. Clin Cancer Res 2018;24:4282-4296. 
Supplementary Table 1. Human primers used for determination of $C D H 1$ and ZEB1 correlation in the Rennes cohort by quantitative real-time PCR.

\begin{tabular}{ccccc}
\hline Gene & Protein & Forward $\left(5^{\prime} \rightarrow 3^{\prime}\right)$ & Reverse $\left(5^{\prime} \rightarrow 3^{\prime}\right)$ & Accession Number \\
\hline CDH1 & E-cadherin & CGAGAGCTACACGTTCACGG & GGGTGTCGAGGGAAAAATAGG & NM_004360.4 \\
TBP & TBP & GAGCTGTGATGTGAAGTTTCC & TCTGGGTTTGATCATTCTGTAG & NM_003194.5 \\
ZEB1 & ZEB1 & CCCACACGACCACAGATACG & TGAGGAGAACTGGTTGCCTG & NM_001128128.2 \\
\hline
\end{tabular}

Supplementary Table 2. Mouse primers used for quantitative real-time PCR.

\begin{tabular}{ccccc}
\hline Gene & Protein & Forward $\left(5^{\prime} \rightarrow 3^{\prime}\right)$ & Reverse $\left(5^{\prime} \rightarrow 3^{\prime}\right)$ & Accession Number \\
\hline Acta2 & a-SMA & CTGTCAGGAACCCTGAGACGCT & TACTCCCTGATGTCTGGGAC & NM_007392.3 \\
Adgre1 & F4/80 & CTTTGGCTATGGGCTTCCAGTC & GCAAGGAGGACAGAGTTTATCGTG & NM_010130.4 \\
Ccl2 & Mcp1 & GCCTGCTGTTCACAGTTGC & CAGGTGAGTGGGGCGTTA & NM_011333 \\
Ccn2 & Ctgf & GGGCCTCTTCTGCGATTTC & ATCCAGGCAAGTGCATTGGTA & NM_010217.2 \\
Col1a1 & Collagen I & GAAACCCGAGGTATGCTTGA & GACCAGGAGGACCAGGAAGT & NM_007742.4 \\
Col4a1 & Collagen IV & ATTTCCAGGCGTGTCATTGC & AGCTCTCTCCTTTCTGACCTT & NM_009931.2 \\
Egf & Egf & TGCCTCAGAAGGAGTGGGTTA & GTGTTCCAAGCGTTCCTGAGA & NM_010113.4 \\
Hgf & Hgf & ATGTGGGGGACCAAACTTCTG & GGATGGCGACATGAAGCAG & NM_001289458.1 \\
Hprt & Hprt & TCAGTCAACGGGGGACATAA & TGCTTAACCAGGGAAAGCAAA & NM_013556.2 \\
lgf2 & Igf2 & GCATCGTGGAAGAGTGCTG & GGGTATCTGGGGAAGTCGTC & NM_001315489.1 \\
Il6 & Il6 & TAGTCCTTCCTACCCCAATTTCC & TTGGTCCTTAGCCACTCCTTC & NM_031168.2 \\
Pdgfb & Pdgfb & CATCCGCTCCTTTGATGATCTT & GTGCTCGGGTCATGTTCAAGT & NM_011057.4 \\
Pecam1 & Cd31 & AGCCTCCAGGCTGAGGAAA & GATGTCCACAAGGCACTCCA & NM_001305158.1 \\
Tgfb1 & TgfB1 & CTCCCGTGGCTTCTAGTGC & GCCTTAGTTTGGACAGGATCTG & NM_011577.2 \\
Vwf & Vwf & GCTGCAGTTATCTCCTGGCT & ACAGGCTCATTCTCTTGCCA & NM_011708.4 \\
Zeb1 & Zeb1 & CAGATTCCGATGATGAAGACAAAC & TCTTTCACGTTGTCTTGCCAGC & NM_011546.3 \\
\hline
\end{tabular}


Supplementary Table 3. Human primers used for quantitative real-time PCR.

\begin{tabular}{|c|c|c|c|c|}
\hline Gene & Protein & Forward (5’ $\left.\rightarrow 3^{\prime}\right)$ & Reverse $\left(5^{\prime} \rightarrow 3^{\prime}\right)$ & Accession Number \\
\hline ACTA2 & $\alpha-S M A$ & GACAATGGCTCTGGGCTCTGTAA & CTGTGCTTCGTCACCCACGTA & NM_001141945.1 \\
\hline CCN2 & CTGF & CAGCATGGACGTTCGTCTG & AACCACGGTTTGGTCCTTGG & NM_001901.3 \\
\hline$C D 24$ & CD24 & CTCCTACCCACGCAGATTTATTC & AGAGTGAGACCACGAAGAGAC & NM_013230.3 \\
\hline$C D 44$ & CD44 & TGCAGTCAACAGTCGAAGAAG & CTGTCCTCCACAGCTCCATT & NM_000610.4 \\
\hline $\mathrm{CDH} 1$ & E-Cadherin & AAGAAGCTGGCTGACATGTACGGA & CCACCAGCAACGTGATTTCTGCAT & NM_004360.4 \\
\hline COL1A1 & Collagen I & AGTTCGAGTATGGCGG & CAGTGACGCTGTAGGT & NM_000088.3 \\
\hline COL4A1 & Collagen IV & CCGCTGCGAAGGGTGG & CCCTTTTGTCCTGGTGGTCCC & NM_001845.5 \\
\hline CXCL8 & IL8 & ACTGAGAGTGATTGAGAGTGGAC & AАCCCTCTGCACCCAGTTTTC & NM_000584 \\
\hline$E G F$ & EGF & AGTGCATCCACTTGCACAAC & CAATTTGCAAAGTTTCTGCTCA & NM_001963.4 \\
\hline EPCAM & EpCAM & CCATGTGCTGGTGTGTGAA & TGTGTTTTAGTTCAATGATGATCCA & NM_002354.2 \\
\hline ESRP1 & ESRP1 & CCCACCGCCATGTAAGTT & GCAGGAGCTGGAAATGTGTAG & NM_017697.4 \\
\hline GAPDH & GAPDH & AGCCACATCGCTCAGACAC & GCCCAATACGACCAAATCC & NM_002046.5 \\
\hline HGF & HGF & AAAGGACTTCCATTCACTTGC & CCACTCTTAGTGATAGATACTG & NM_000601.4 \\
\hline IGF2 & IGF2 & CGTCGCAGCCGTGGCATCGTTGA & GCCCACGGGGTATCTGGGGAAG & NM_000612.5 \\
\hline IL6 & IL6 & TACATCCTCGACGGCATCTC & TCCAGATTGGAAGCATCCATCCATC & NM_000600.5 \\
\hline IL6R & IL6R & ССССTCAGCAATGTTGTTTGT & CTCCGGGACTGCTAACTGG & NM_001206866.2 \\
\hline IL6ST & GP130 & CGGACAGCTTGAACAGAATGT & АССАТСССАСТСАСАССТСА & NM_002184.4 \\
\hline ITGB3 & CD61 & CAAGATTGGAGACACGGTGA & CGGGCTTTATGGTAAAGGACT & NM_000212.3 \\
\hline$M E T$ & MET & AGCAATGGGGAGTGTAAAGAGG & CCCAGTCTTGTACTCAGCAAC & NM_000245.4 \\
\hline SNAl1 & SNAIL & CCAGAGTTTACCTTCCAGCAGCC & CAGGACAGAGTCCCAGATGAGCAT & NM_005985.3 \\
\hline$P D G F B$ & PDGFB & GGGCATCGGCAGGAGAGT & AGACAGACGGACGAGGGAAA & NM_002608.2 \\
\hline SNAI2 & SLUG & ATCTCCGGAGGTGGGATG & GAGAGACATTCTGGAGAAGG & NM_003068.4 \\
\hline TGFB1 & TGFB1 & GCCCTGGACACCAACTATTGC & GGCTCCAAATGTAGGGGCAGG & NM_000660.5 \\
\hline TWIST1 & TWIST1 & AGCTACGCCTTCTCGGTCT & ССTTCTCTGGAAACAATGACATC & NM_000474.3 \\
\hline TWIST2 & TWIST2 & GCAAGAAGTCGAGCGAAGAT & GCTCTGCAGCTCCTCGAA & NM_001271893.4 \\
\hline VIM & Vimentin & CCCTGCAATCTTTCAGACAG & ATCTGAGCCTGCAGCTCCT & NM_003380.3 \\
\hline ZEB1 & ZEB1 & ACAATCGTGGCCATTGCTGA & TGGTTCTTGGACTGCAGGGCT & NM_001128128.2 \\
\hline ZEB2 & ZEB2 & AGGGACAGATCAGCACCAAATGCTA & GGCACTCGTAAGGTTTTTCACCACTG & NM_014795.3 \\
\hline
\end{tabular}


Supplementary Table 4. Human primers used for chromatin immunoprecipitation PCR (CHIP-PCR) experiments.

\begin{tabular}{cccc}
\hline Gene & Forward $\left(5^{\prime} \rightarrow 3^{\prime}\right)$ & Reverse $\left(5^{\prime} \rightarrow 3^{\prime}\right)$ & Accession Number \\
\hline CCN2 & GCTGGAGTGTGCCAGCTTT & CGAGGCTTTTATACGCTCCG & NG_016131.1 \\
HGF & GGGTAAGGGAGCTGGAGGACAA & AGAACAGTGCCCCTGTCCA & NG_016274.2 \\
\hline
\end{tabular}

Supplementary Table 5. Primary antibodies used for immunodetection.

\begin{tabular}{|c|c|c|c|c|c|}
\hline Name & Species & Manufacturer & Reference & Dilution & Antigen unmasking \\
\hline$\alpha-S M A$ & $M$ & Dako & M851 & $1 / 500(\mathrm{IHC})$ & \\
\hline pAKT & $\mathrm{R}$ & Cell Signaling & \#4060 & $1 / 1000$ (WB) & \\
\hline AKT & $\mathrm{R}$ & Cell Signaling & \#4691 & 1/1000 (WB) & \\
\hline CTGF & $\mathrm{M}$ & Santa Cruz & Sc-365970 & 1/1000 (WB) & \\
\hline E-cadherin & M & Abcam & ab1416 & 1:5000 (WB) / 1:250 (IF) & \\
\hline pERK & $\mathrm{M}$ & Santa Cruz & sc-7383 & $1: 250(\mathrm{WB})$ & \\
\hline ERK 1 & $\mathrm{R}$ & Santa Cruz & sc-93 & $1: 500(\mathrm{WB})$ & \\
\hline GAPDH & $\mathrm{M}$ & Santa Cruz & sc-32233 & 1:5000 (WB) & \\
\hline SLUG & $\mathrm{R}$ & Cell Signaling & \#9585 & 1/1000 (WB) & \\
\hline SNAIL & $\mathrm{R}$ & Cell Signaling & \#3879 & 1/1000 (WB) & \\
\hline pSTAT3 & $\mathrm{R}$ & Cell Signaling & \#9145 & 1:500 (WB) & \\
\hline STAT3 & $\mathrm{R}$ & Cell Signaling & \#4904 & $1: 500(\mathrm{WB})$ & \\
\hline TWIST1 & $\mathrm{M}$ & Santa Cruz & sc-81417 & 1:500 (WB) & \\
\hline TWIST2 & $\mathrm{R}$ & Abcam & $a b 66031$ & 1:1000 (WB) & \\
\hline Vimentin & $\mathrm{M}$ & Sigma & V6630 & $1: 15000$ (WB) & \\
\hline ZEB1 & $\mathrm{R}$ & Sigma & HPA027524 & 1/200 (IHC) & yes \\
\hline ZEB1 & $\mathrm{R}$ & Santa Cruz & sc-25388 & 1:500 (WB) & \\
\hline ZEB2 & $\mathrm{R}$ & Santa Cruz & sc-48789 & 1:500 (WB) & \\
\hline
\end{tabular}

$M$, mouse; R, rabbit; WB, western blot; IF, immunofluorescence; IHC, immunohistochemistry. 
Supplementary Table 6. Clinical and pathological characteristics of patients with CCA $(n=45)$

\begin{tabular}{lc}
\hline $\begin{array}{l}\text { Age (years) } \\
\text { Mean }( \pm \mathrm{SD})\end{array}$ & $62+12$ \\
\hline Sex ratio (M/F) & 0.9 \\
\hline Tumor size (mm) & $82(15-220)$ \\
$\quad$ Mean $( \pm \mathrm{SD})$ & \\
\hline Tumor grade & $17 / 45(38 \%)$ \\
Well differentiated & $18 / 45(40 \%)$ \\
Moderately differentiated & $10 / 45(22 \%)$ \\
Poorly differentiated & \\
\hline pTNM $\left(7^{\text {th }}\right.$ edition) & $13 \%$ \\
T1a & $9 \%$ \\
T1b & $78 \%$ \\
T2 & $71 \%$ \\
\hline Vascular invasion & $31 \%$ \\
\hline Perineural invasion & $37 \%$ \\
\hline Lymph node metastasis &
\end{tabular}


ZEB1

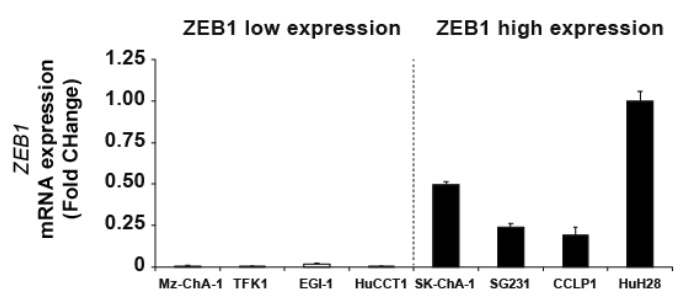

\section{EPITHELIAL MARKERS}
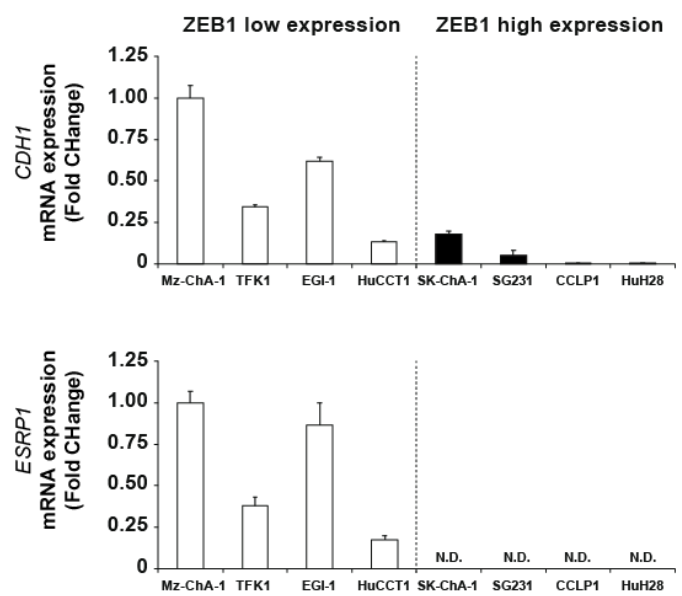

MESENCHYMAL MARKER

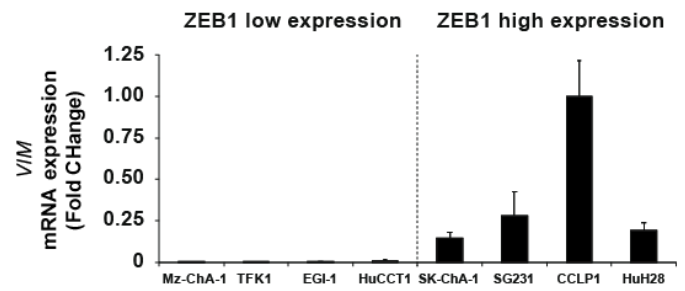

EMT-TF
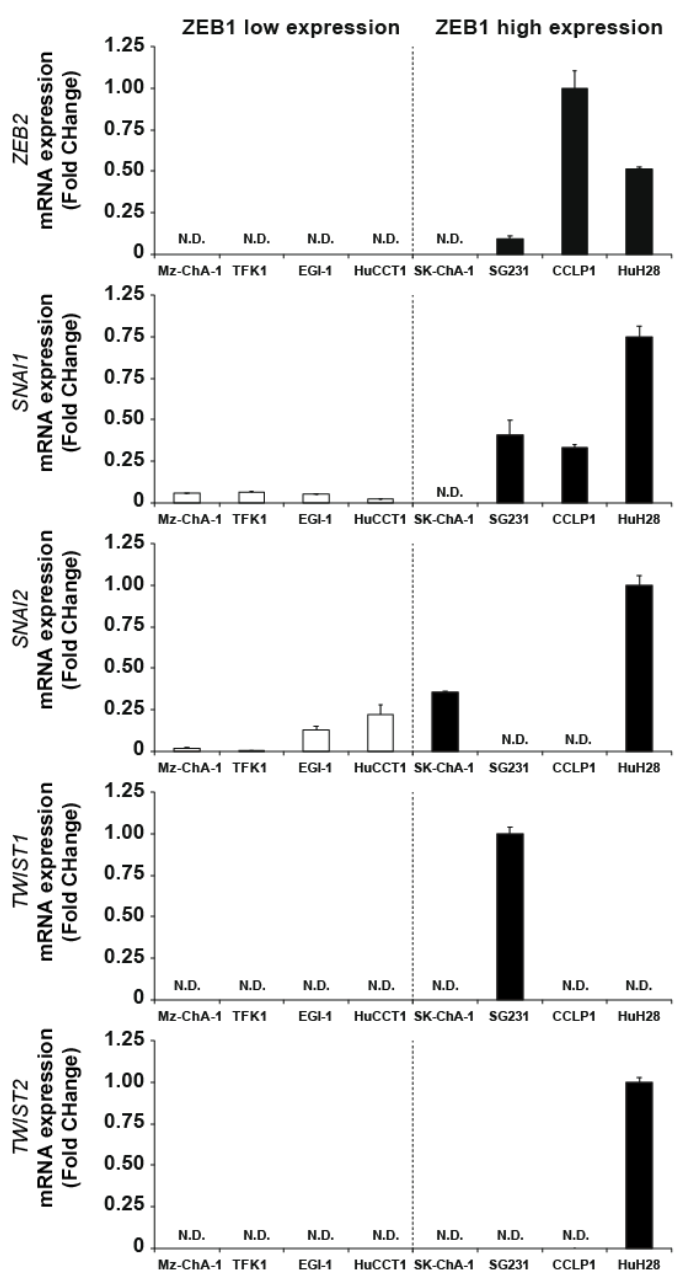

Supplementary Figure 1. ZEB1 expression correlates with that of EMT markers. EMT markers CDH1 (E-cadherin), ESRP1 and VIM (vimentin) and EMT-TFs ZEB2, SNAI1 (SNAIL), SNAI2 (SLUG), TWIST1 and TWIST2 mRNA expression in a panel of 8 human CCA cell lines with epithelial morphology and negligible ZEB1 expression (white bars) or mesenchymal morphology and high ZEB1 expression (black bars). Values are expressed as means \pm SEM from 5 independent cultures. 

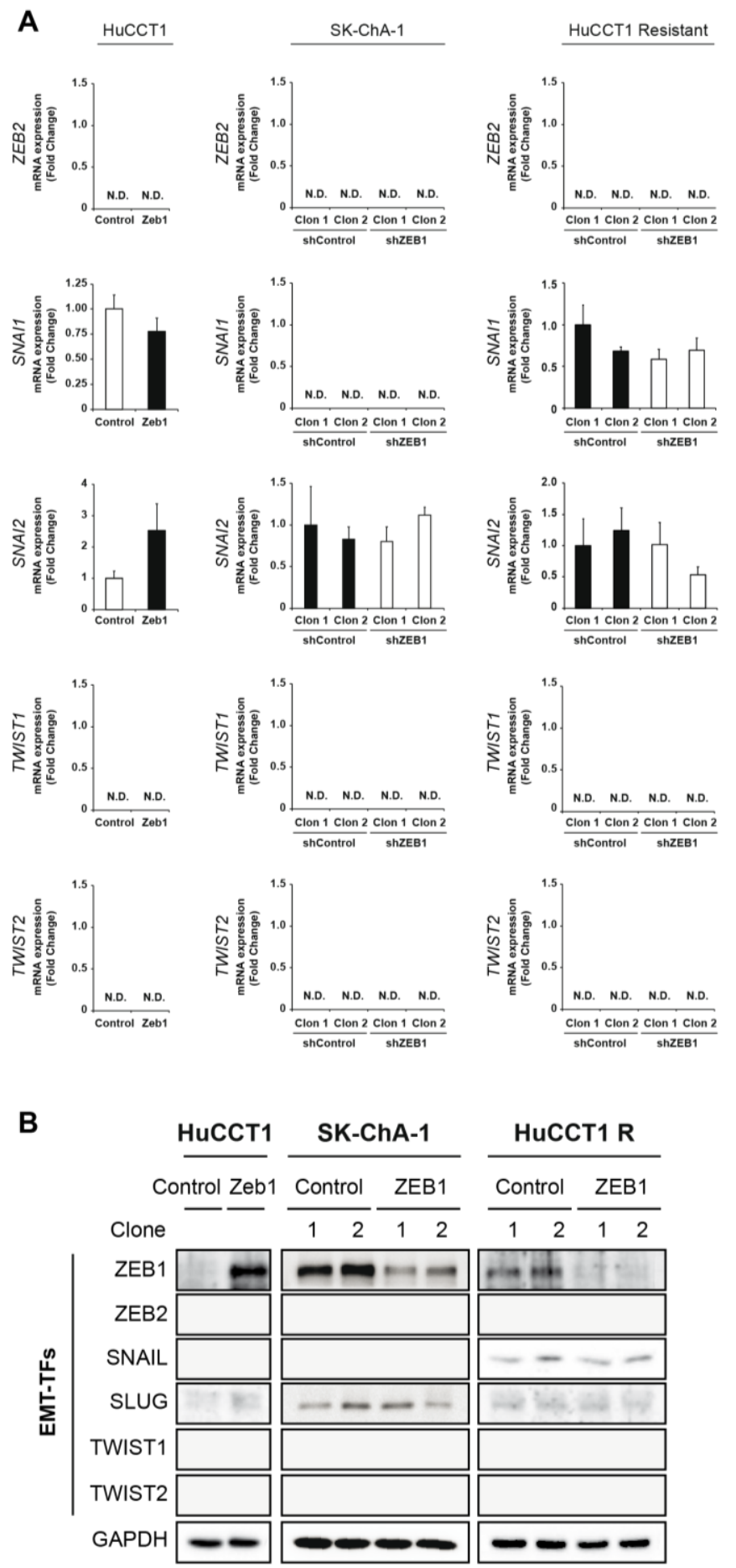

Supplementary Figure 2. ZEB1 gain/loss of function in in vitro models do not modify the expression of other EMT-TFs as a compensatory mechanism. A-B. Changes in EMT-TFs ZEB2, SNAI1 (SNAIL), SNAI2 (SLUG), TWIST1 and TWIST2 mRNA $(A)$ and protein (B) expression in gain/loss of function models derived from HuCCT1, SK-ChA-1 and HuCCT1 R cells, as determined by RT-QPCR and western blot. Values are expressed as means \pm SEM from 5 independent cultures. ND, Not Detected. 

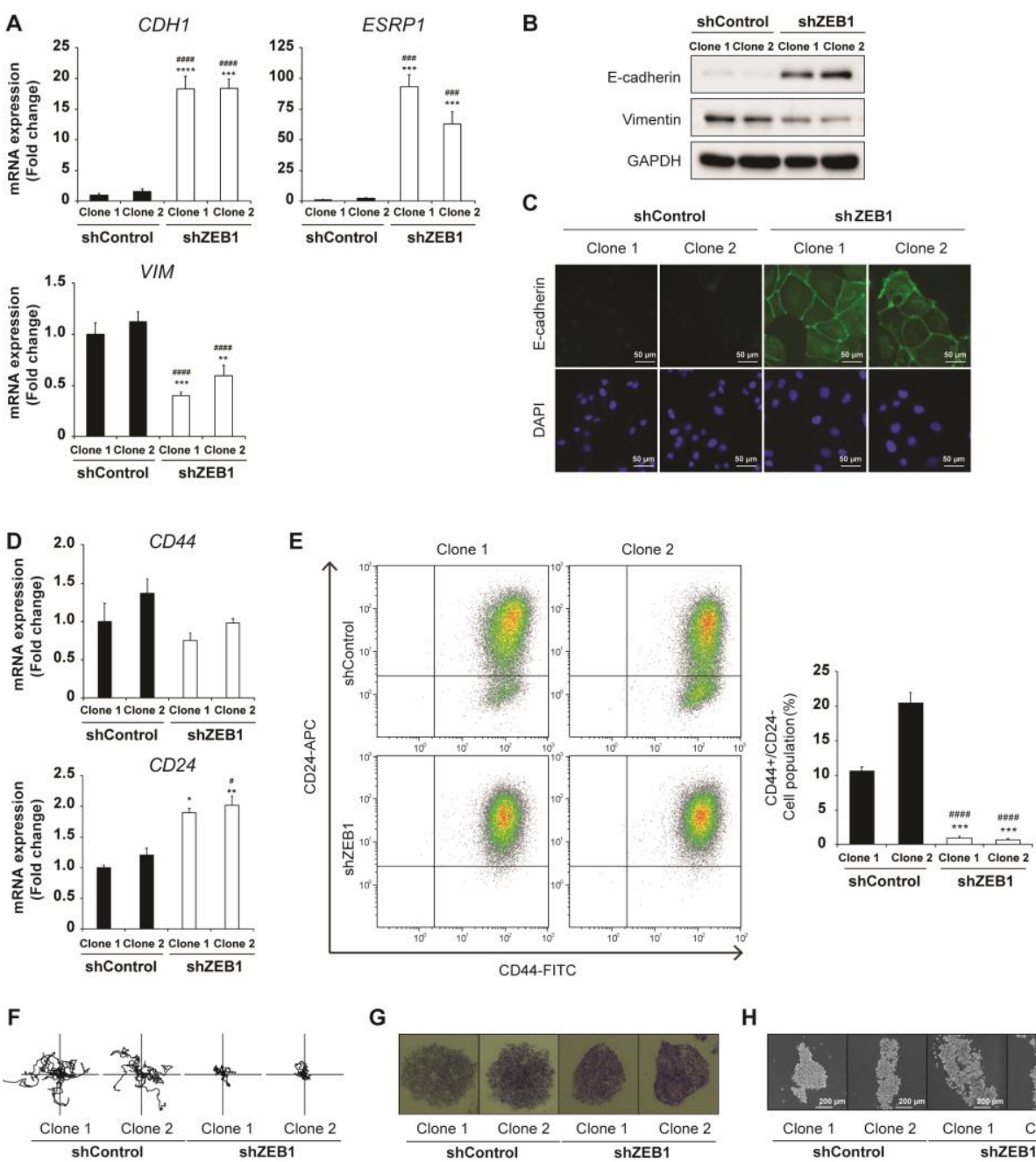

G

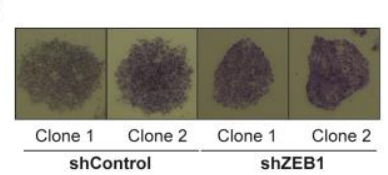

H
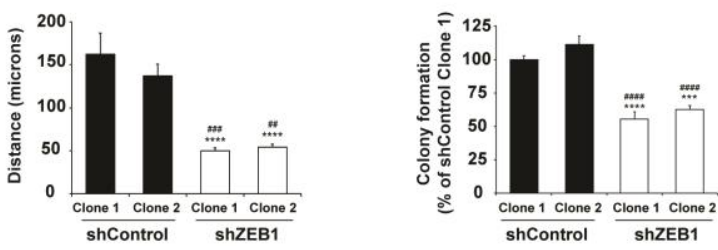

Supplementary Figure 3. ZEB1 expression promotes the acquisition of an EMT/CSC phenotype in CCA cells. A-C. Changes in CDH1 (E-cadherin), ESPR1, and VIM (vimentin) mRNA (A), and protein expression (B) and localization (C) of E-cadherin and vimentin in SK-ChA-1 cells downregulated or not for ZEB1 by shRNA by shRNA, as determined by RT-QPCR, western blot and immunofluorescence. Scale: $50 \mu \mathrm{m}$. D-E. Changes in CD44 and CD24 mRNA and protein expression in the same cells, as determined by RT-QPCR (D) and by flow cytometry (E) using APC-conjugated antiCD24 and FITC-conjugated anti-CD44 antibodies. Gates are based on the isotype controls. Quantification in the right panel shows the \% of cells in the lower right quadrant corresponding to a CD44 $4^{+}$CD24 cell population. F. Cell tracking analyzed by time-lapse microscopy during a $24 \mathrm{~h}$ long time-lapse experiment. Tracks of 8 representative cells for each condition are shown. Bar graphs show quantification of the distance travelled by the cells. G. Colony formation assay after 7 days in culture. Representative pictures of colonies from each cell line. H. Sphere formation assay after 7 days in culture. Representative pictures of spheres from each cell line. SK-ChA-1 sphere counting was impossible due to aggregate formation. Scale: $200 \mu \mathrm{m}$. Values are expressed as means \pm SEM from at least 3 independent cultures. ${ }^{*}, p<0.05 ;{ }^{* *}, p<0.01 ;{ }^{* * *}, p<0.001,{ }^{* * \star *}$, $p<0.0001$, as compared with shControl clone 1. \#, $p<0.05$; \#\#, $p<0.01$; \#\#\#, $p<0.001$; $\# \# \#$, $\mathrm{p}<0.0001$, as compared with shControl clone 2. 

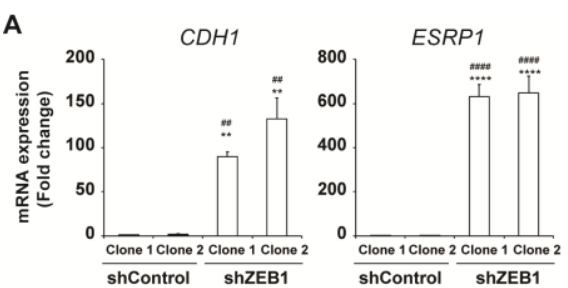

B
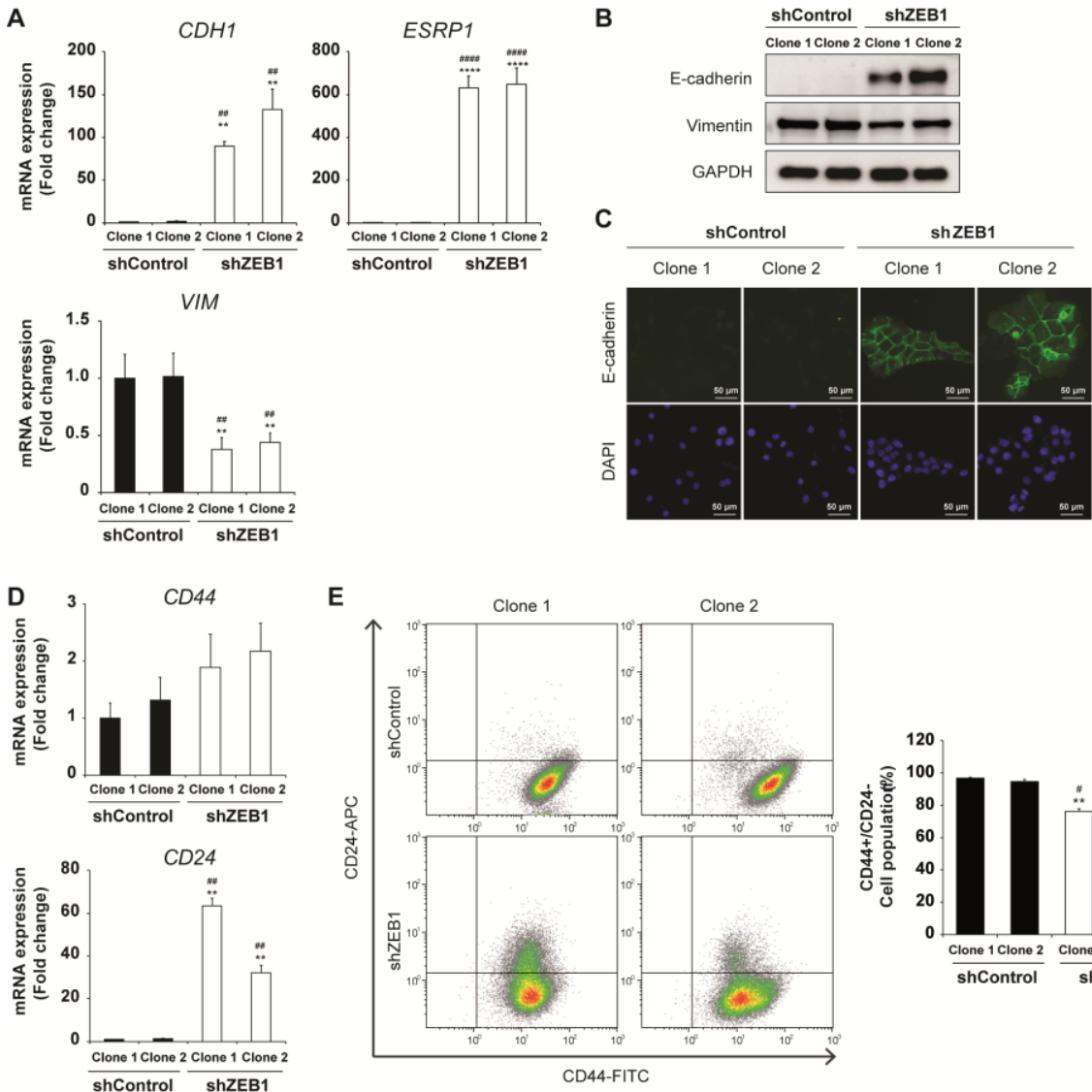

E
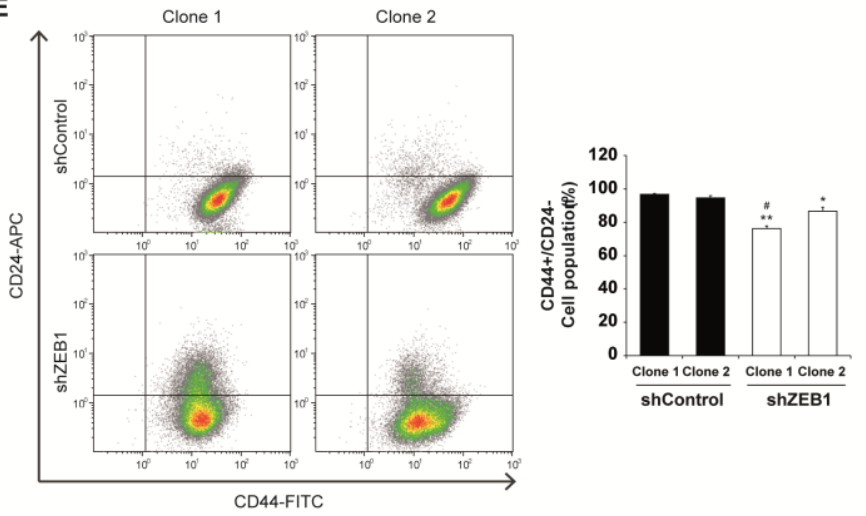

G
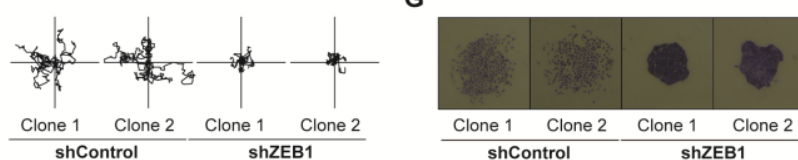

H
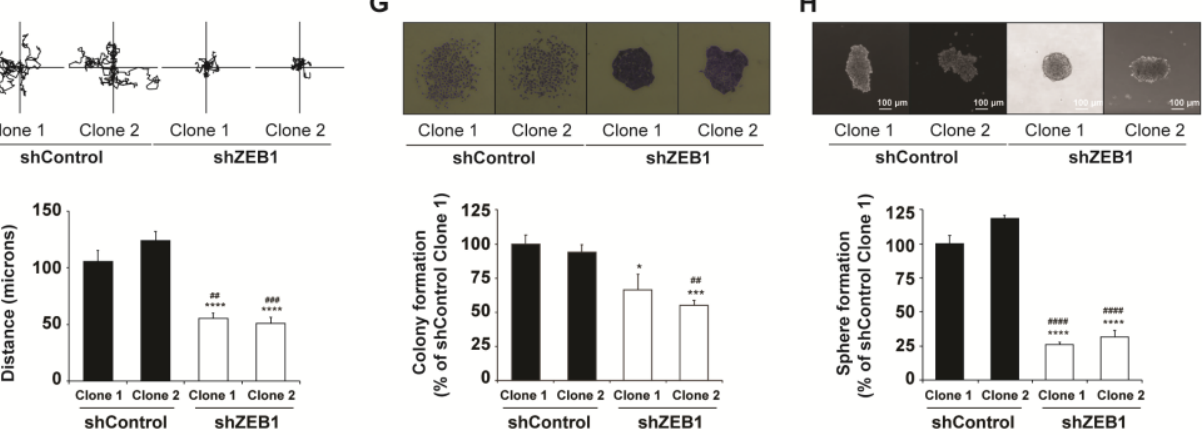

Supplementary Figure 4. ZEB1 expression promotes the acquisition of an EMT/CSC phenotype in CCA cells. A-C. Changes in CDH1 (E-cadherin), ESPR1, and VIM (vimentin) mRNA (A), and protein expression (B) and localization (C) of E-cadherin and vimentin in HuCCT1 $R$ cells downregulated or not for ZEB1 by shRNA, as determined by RT-QPCR, western blot and immunofluorescence. Scale: $50 \mu \mathrm{m}$. D-E. Changes in CD44 and CD24 mRNA and protein expression in the same cells, as determined by RT-QPCR (D) and by flow cytometry (E) using APC-conjugated antiCD24 and FITC-conjugated anti-CD44 antibodies. Gates are based on the isotype controls. Quantification in the right panel shows the \% of cells in the lower right quadrant corresponding to a CD44 $/ \mathrm{CD} 24$ cell population. F. Cell tracking analyzed by time-lapse microscopy during a $24 \mathrm{~h}$ long time-lapse experiment. Tracks of 8 representative cells for each condition are shown. Bar graphs show quantification of the distance travelled by the cells. G. Colony formation assay after 7 days in culture. Representative pictures of colonies from each cell line. H. Sphere formation assay after 7 days in culture. Representative pictures of spheres from each cell line. Scale: $200 \mu \mathrm{m}$. Values are expressed as means \pm SEM from at least 3 independent cultures. ${ }^{*}, p<0.05 ;{ }^{* *}, p<0.01$; ${ }^{* * *}, p<0.001,{ }^{* * \star *}, p<0.0001$, as compared with shControl clone 1. $\#, p<0.05 ; \# \#, p<0.01$; \#\#\#, $\mathrm{p}<0.001$; \#\#\#\#, $\mathrm{p}<0.0001$, as compared with shControl clone 2. 
A
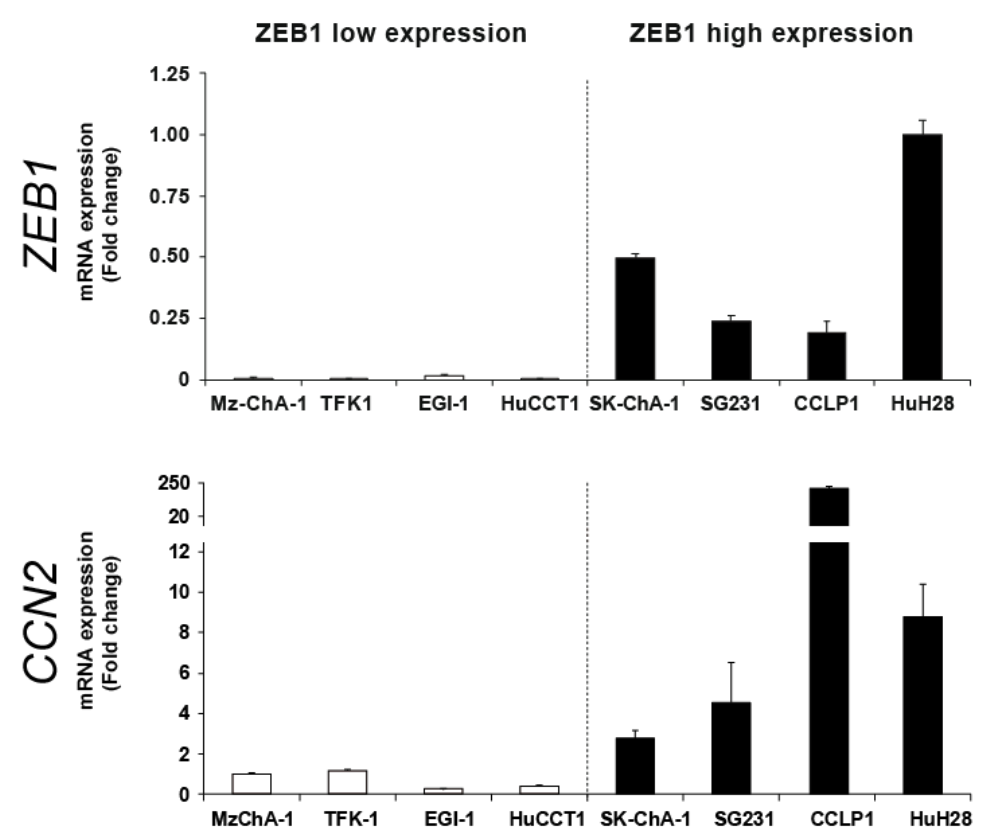

B

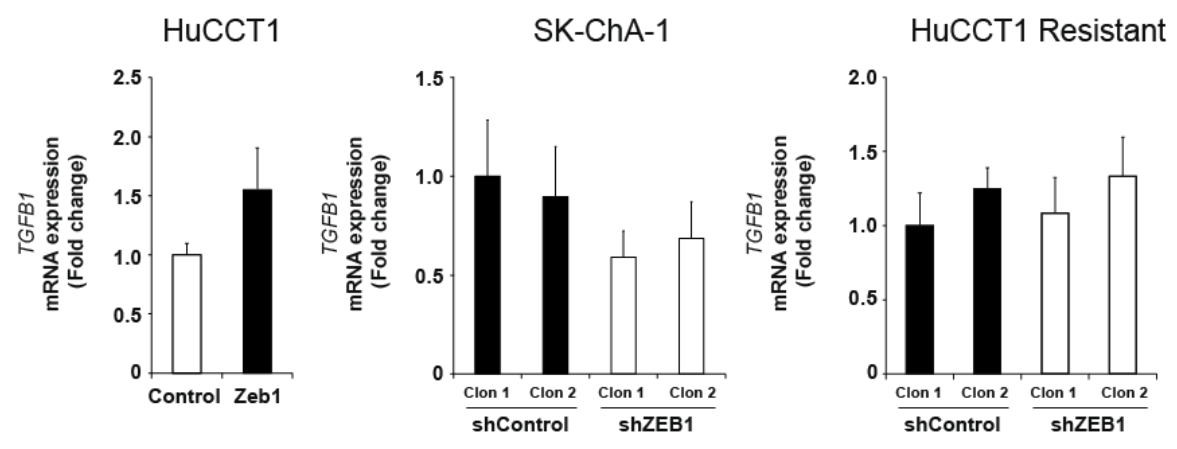

C

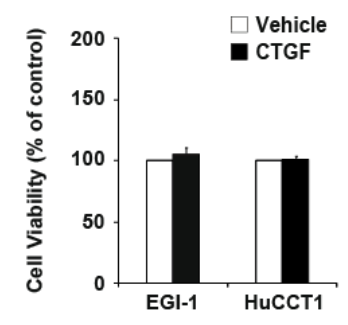

D
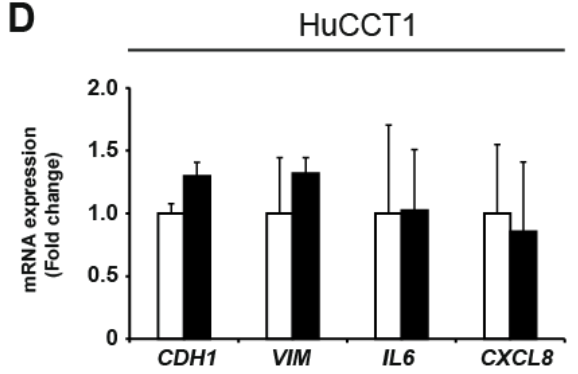

EGI-1

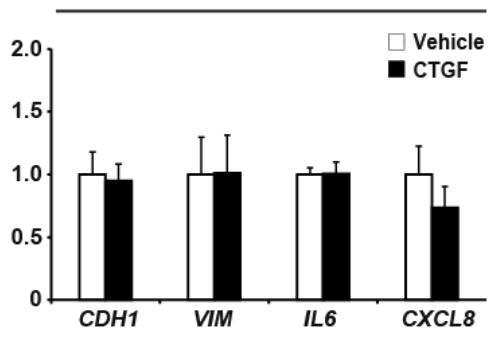

Supplementary Figure 5. A. Changes in CCN2 (encoding CTGF) mRNA expression in a panel of 8 CCA cell lines with epithelial morphology and negligible ZEB1 expression (white bars) or mesenchymal morphology and high ZEB1 expression (black bars). Values are expressed as means \pm SEM from 5 independent cultures. B. Changes in TGFB1 mRNA expression in gain/loss of ZEB1 function models derived from HuCCT1, SK-ChA-1 and HuCCT1 R cells, as determined by RT-QPCR. C-D. Effect of exogenous CTGF $100 \mathrm{ng} / \mathrm{mL}$ on cell viability (C) and mRNA expression of CDH1 (E-cadherin), VIM (vimentin), IL6 and CXCL8 (D) in HuCCT1 and EGl-1 cells. Cell viability and mRNA level were measured after $72 \mathrm{~h}$ and $24 \mathrm{~h}$ of incubation with CTGF, respectively. Values are expressed as means \pm SEM from 5 independent cultures. 


\section{A (Cancer Cell Line Encyclopedia)}

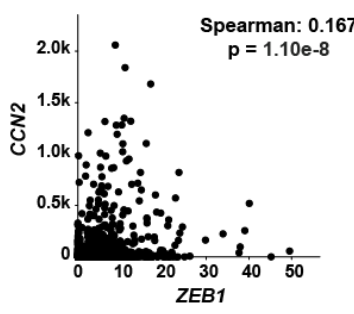

\section{B (TCGA, PanCancer Atlas)}
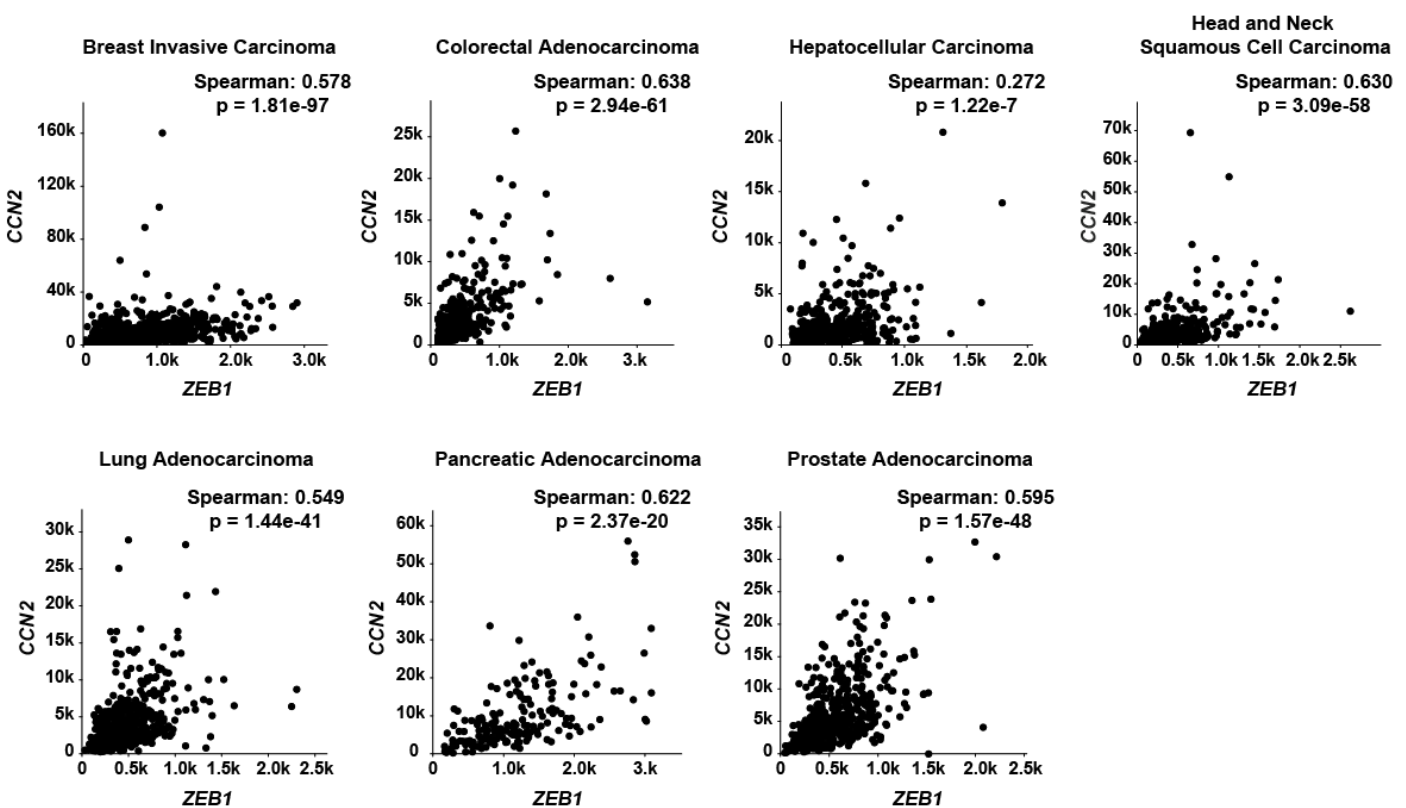

Supplementary Figure 6. CCN2 expression correlates with ZEB1 expression in multiple human cancers and cell lines. A-B. Correlation of $C C N 2$ gene expression with ZEB1 in cell lines from the Cancer Cell Line Encyclopedia $(A)$ and multiple cancers from the TCGA study (B), determined using Spearman's correlation analysis. 
A

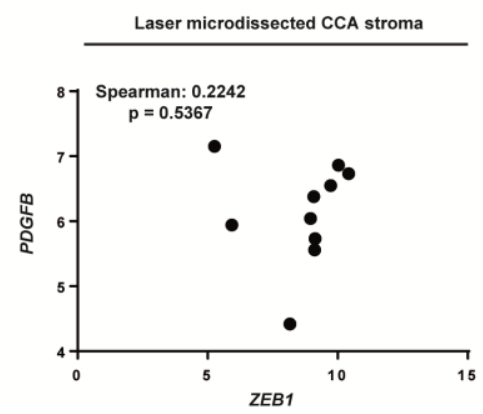

B
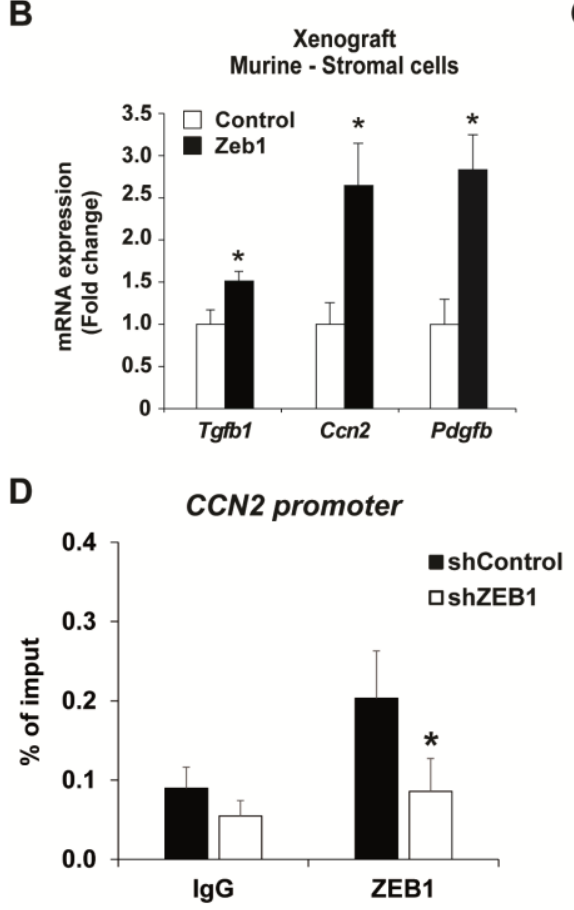

E

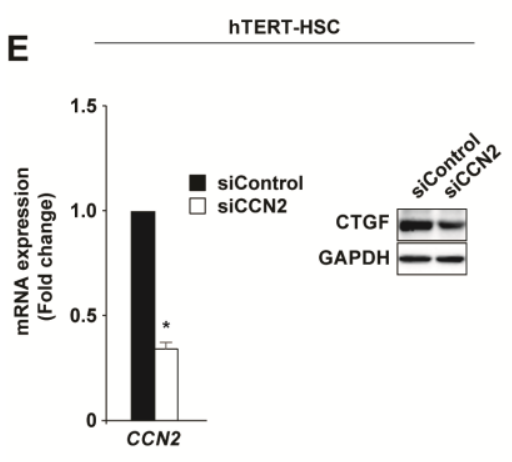

C

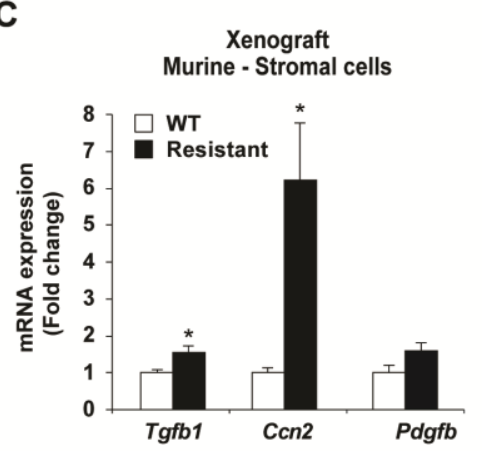

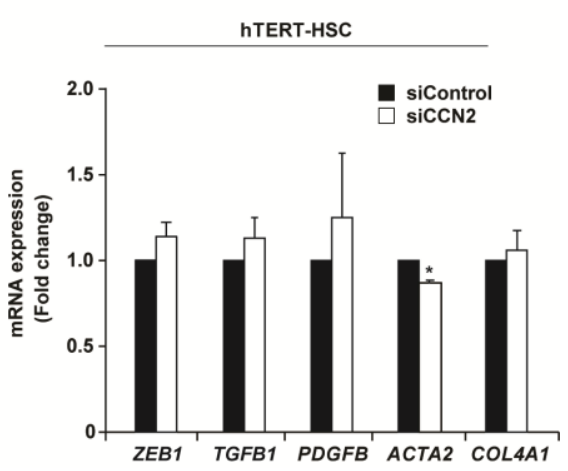

Supplementary Figure 7. A. Correlation between PDGFB with ZEB1 expression in microdissected stroma from 10 iCCA samples determined using Spearman's correlation analysis. B-C. Changes in mRNA expression Tgfb1, Ccn2 and Pdgfb in the stromal compartment (corresponding to murine genes) of xenograft tumors derived from HuCCT1 Control and Zeb1 cells (B), or HuCCT1 WT and Resistant (R) cells (C), analyzed by RT-QPCR. D. Chromatin immunoprecipitation PCR (ChIP-PCR) experiment using primers targeting CCN2 promoter in hTERT-HSC-shControl (black bars) and shZEB1 (white bars) cells. E. Effect of CCN2 downregulation by siRNA transfection in hTERT-HSC cells in the expression of ZEB1, TGFB1, PDGFB, ACTA2 and COL4A1. Values are expressed as means \pm SEM from 5 animals or 5 independent cultures. *, $\mathrm{p}<0.05$ comparing with Control tumors, WT tumors or sh/siControl cells. 
A
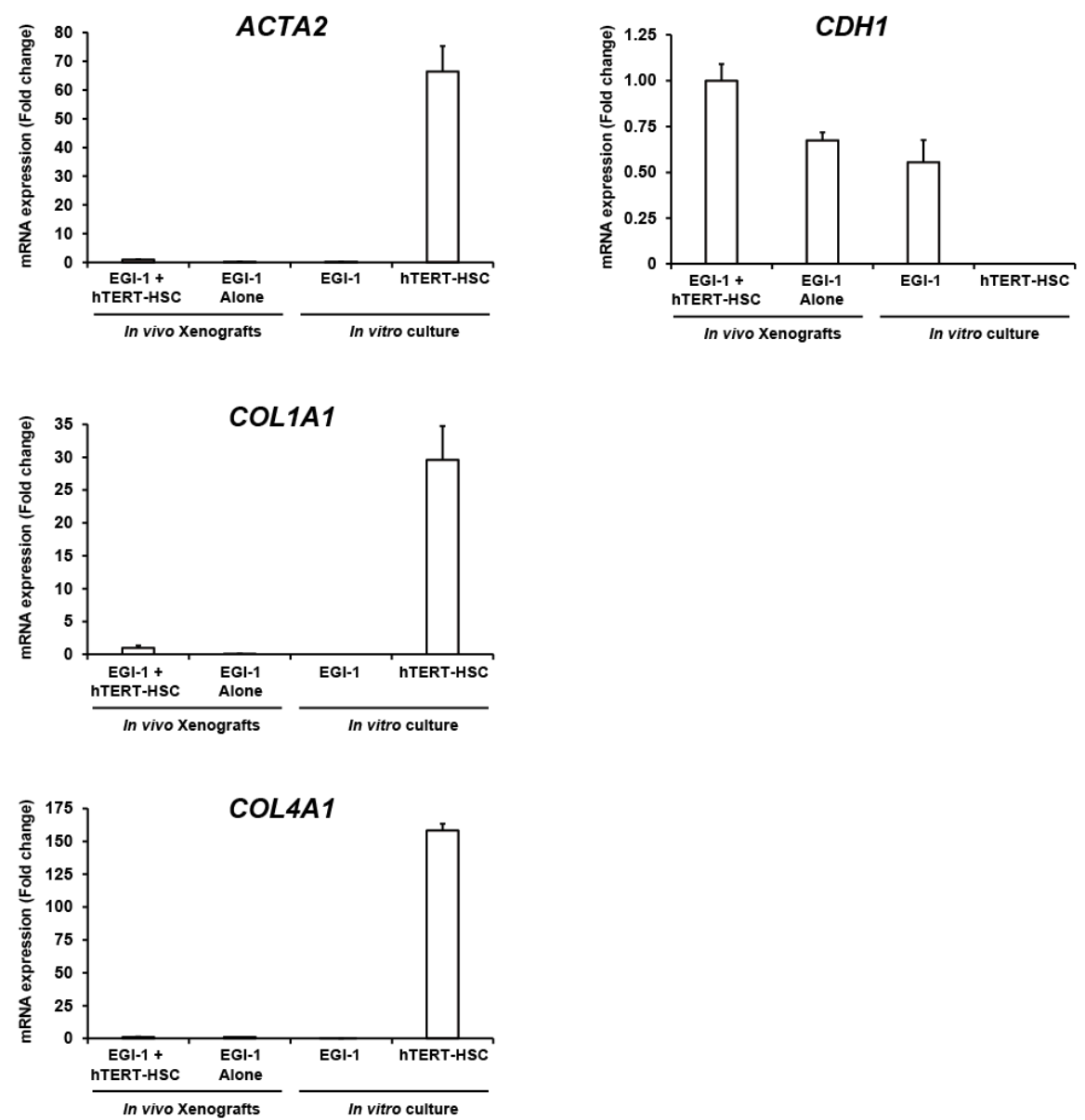

B

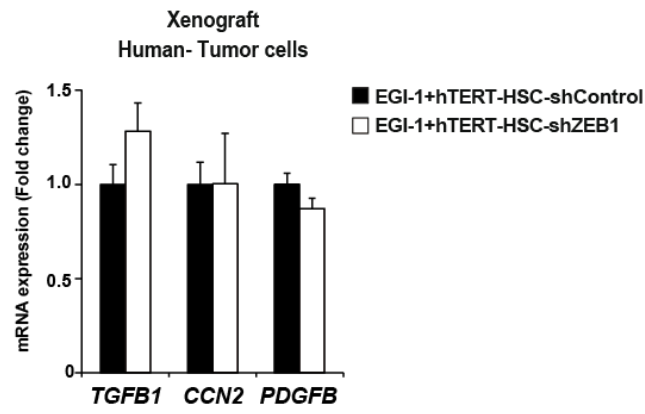

Supplementary Figure 8. A. mRNA expression of human ACTA2 (encoding a-SMA), COL1A1 COL4A1 and CDH1 (encoding E-cadherin) determined by RT-QPCR in xenograft tumors derived from EGI-1+hTERT-HSC or EGI-1 alone, and in EGI-1 and hTERT-HSC cells in vitro. B. mRNA expression of TGFB1, CCN2 and PDGFB in the tumor cells (corresponding to the human genes) of xenograft tumors from mice bearing EGl-1+hTERT-HSC-shControl or EGI-1+hTERT-HSC-shZEB1 cells. Results are expressed as means \pm SEM from 5 animals or 5 independent cultures. 

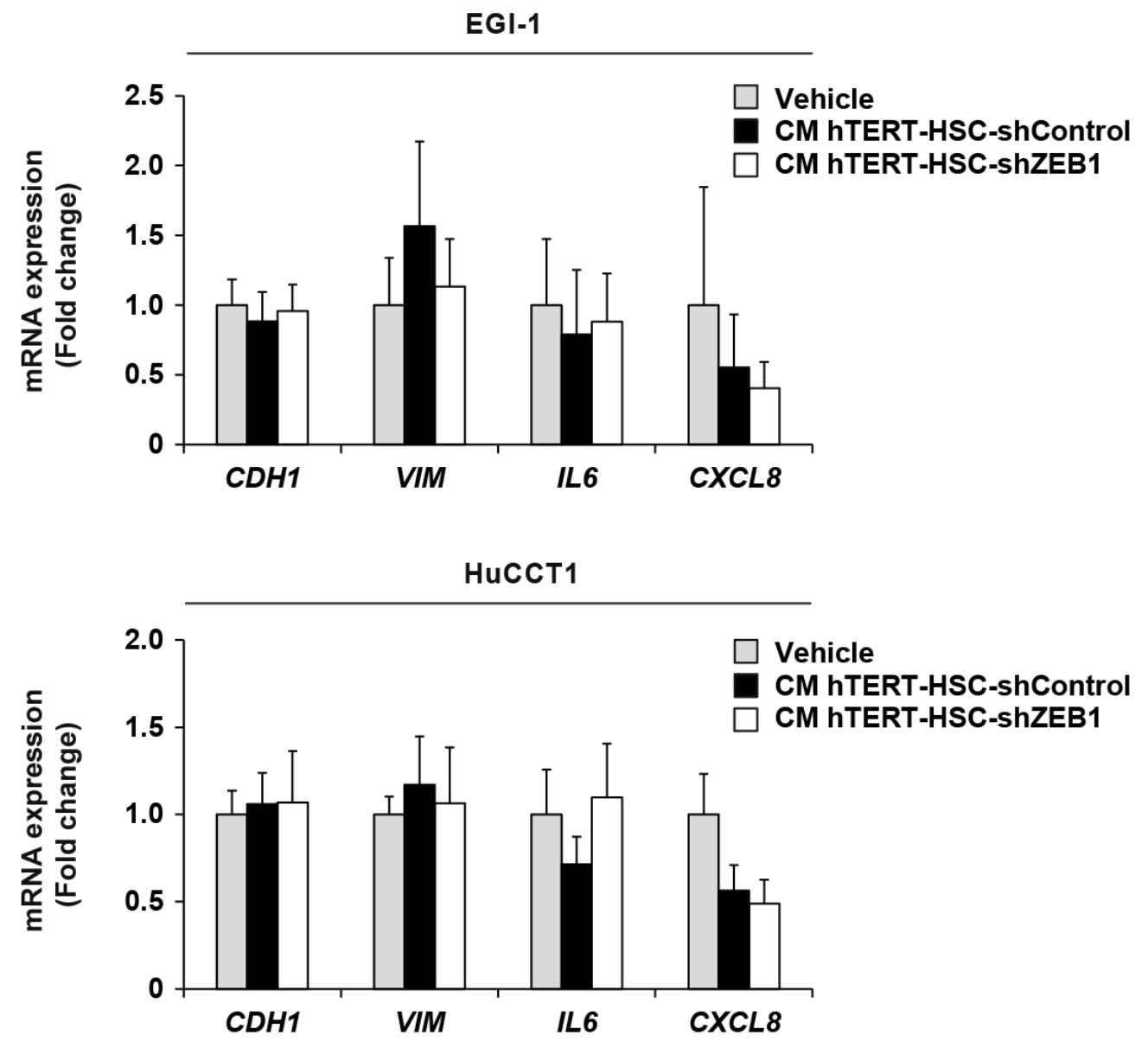

Supplementary Figure 9. Expression of $C D H 1$ (E-cadherin), VIM (vimentin), IL6 and CXCL8 mRNA analyzed by RT-QPCR in EGI-1 or HuCCT1 treated with conditioned media (CM) of hTERT-HSC shControl or shZEB1 cells. Values are expressed as means \pm SEM from 5 independent cultures. 

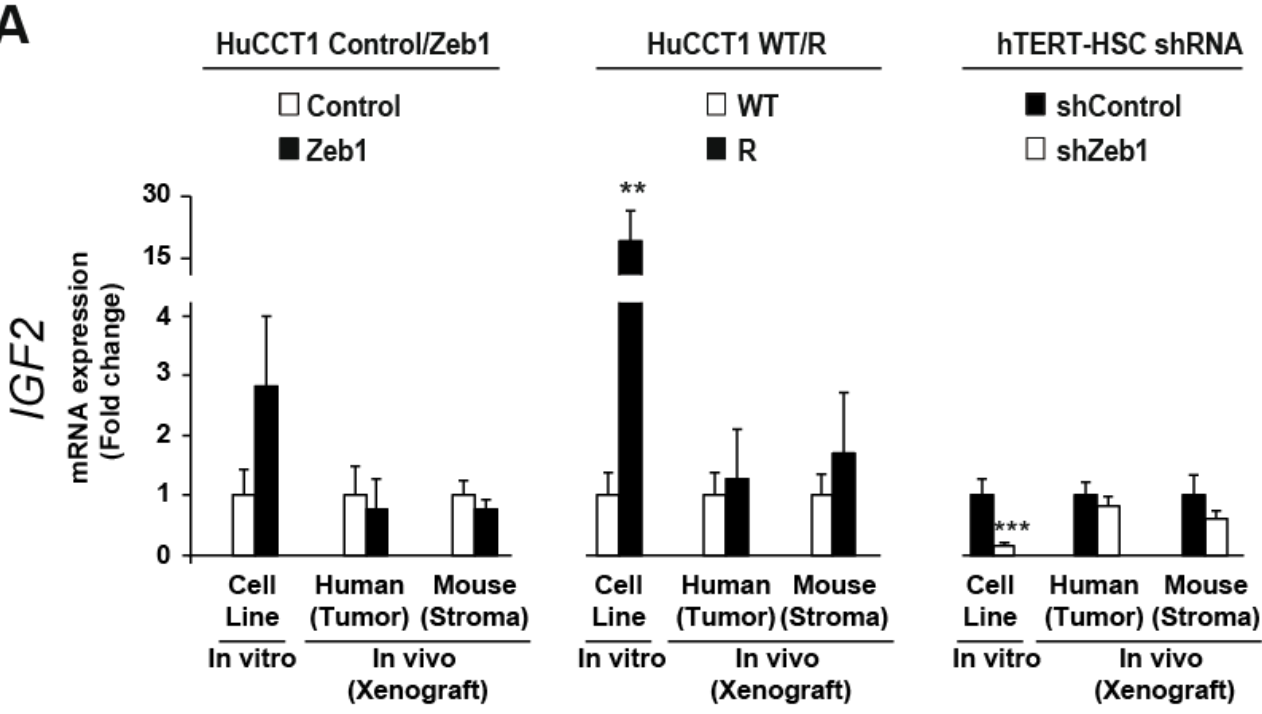

B
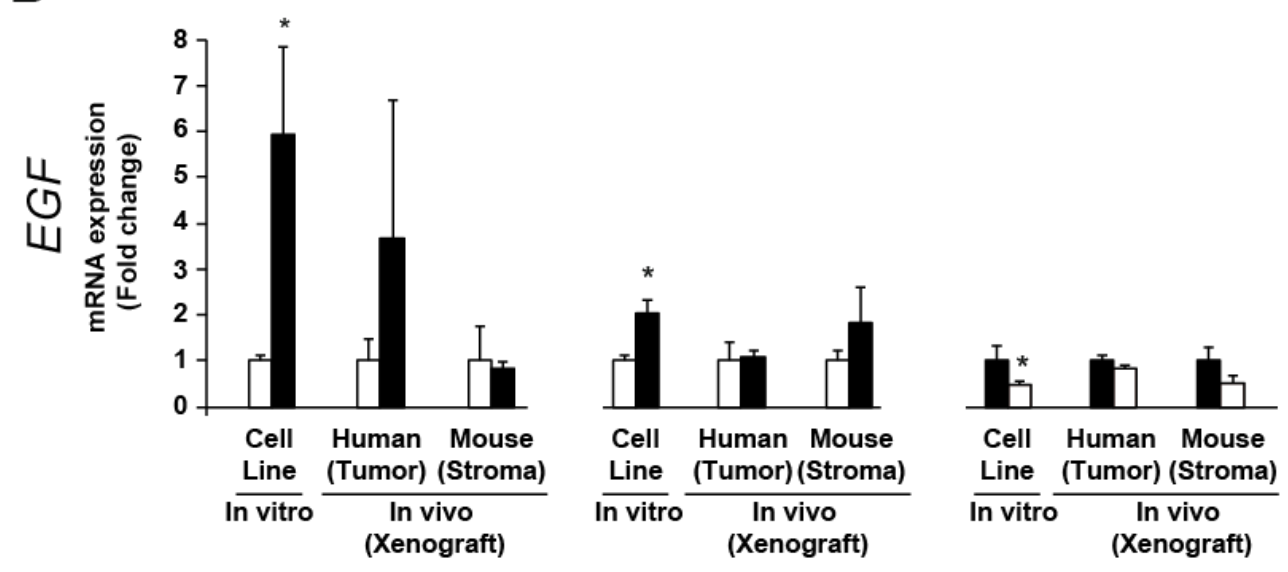

Supplementary Figure 10. A-B. Changes in mRNA expression of IGF2 (A) and EGF (B) in HuCCT1 Control and Zeb1, HuCCT1 WT and Resistant (R) CCA cell lines, and in hTERT-HSC-shRNA-Control and shRNA-ZEB1 cell lines, and their respective derived CCA xenografts, determined by RT-QPCR. For each model gene expression in cultured cells (in vitro), tumor cells (corresponding to human genes) and stromal cells (corresponding to murine genes) of the xenografts (in vivo) is shown. Values are expressed as means \pm SEM from 5 independent cultures/animals. ${ }^{*}, p<0.05 ;{ }^{* *}, p<0.01$; ${ }^{* * *}, \mathrm{p}<0.001$, as compared with Control cells or Control tumors. 
A

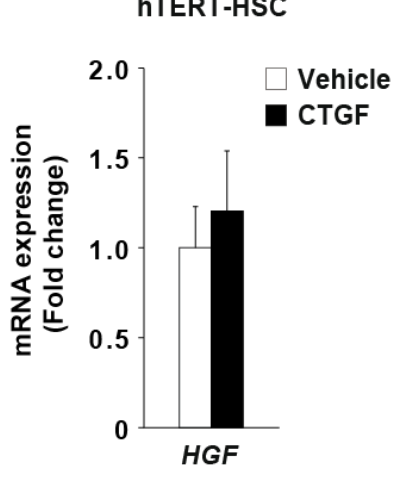

B

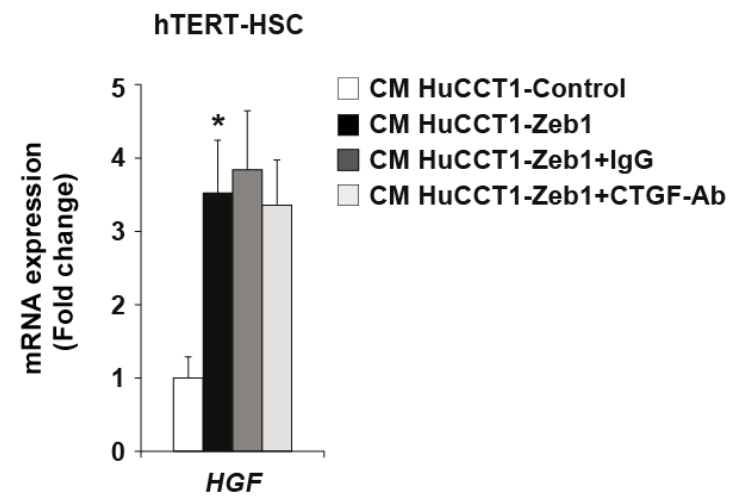

Supplementary Figure 11. Expression of HGF mRNA determined by RT-QPCR in hTERT-HSC cells incubated for $24 \mathrm{~h}$ with recombinant CTGF $100 \mathrm{ng} / \mathrm{mL}(\mathrm{A})$ and conditioned media (CM) (B) from HuCCT1 Control or Zeb1 cells treated with an unspecific IgG or a neutralizing antibody against CTGF. Values are expressed as means \pm SEM from 5 independent cultures. ${ }^{*}, p<0.05$. 
A

Rennes Cohort

CCA

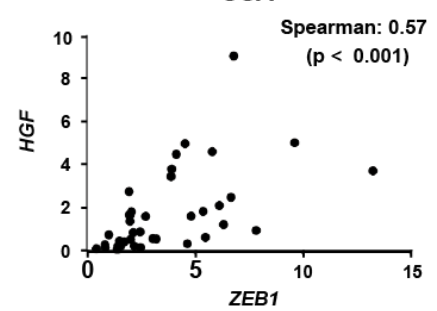

TCGA, PanCancer Atlas CCA

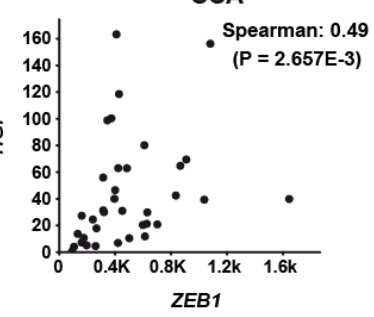

B (Cancer Cell Line Encyclopedia)

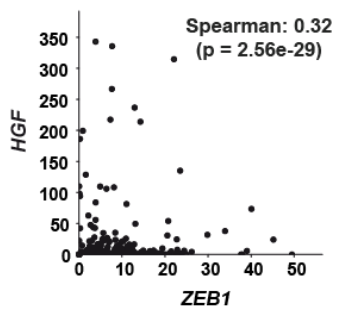

C (TCGA, PanCancer Atlas)
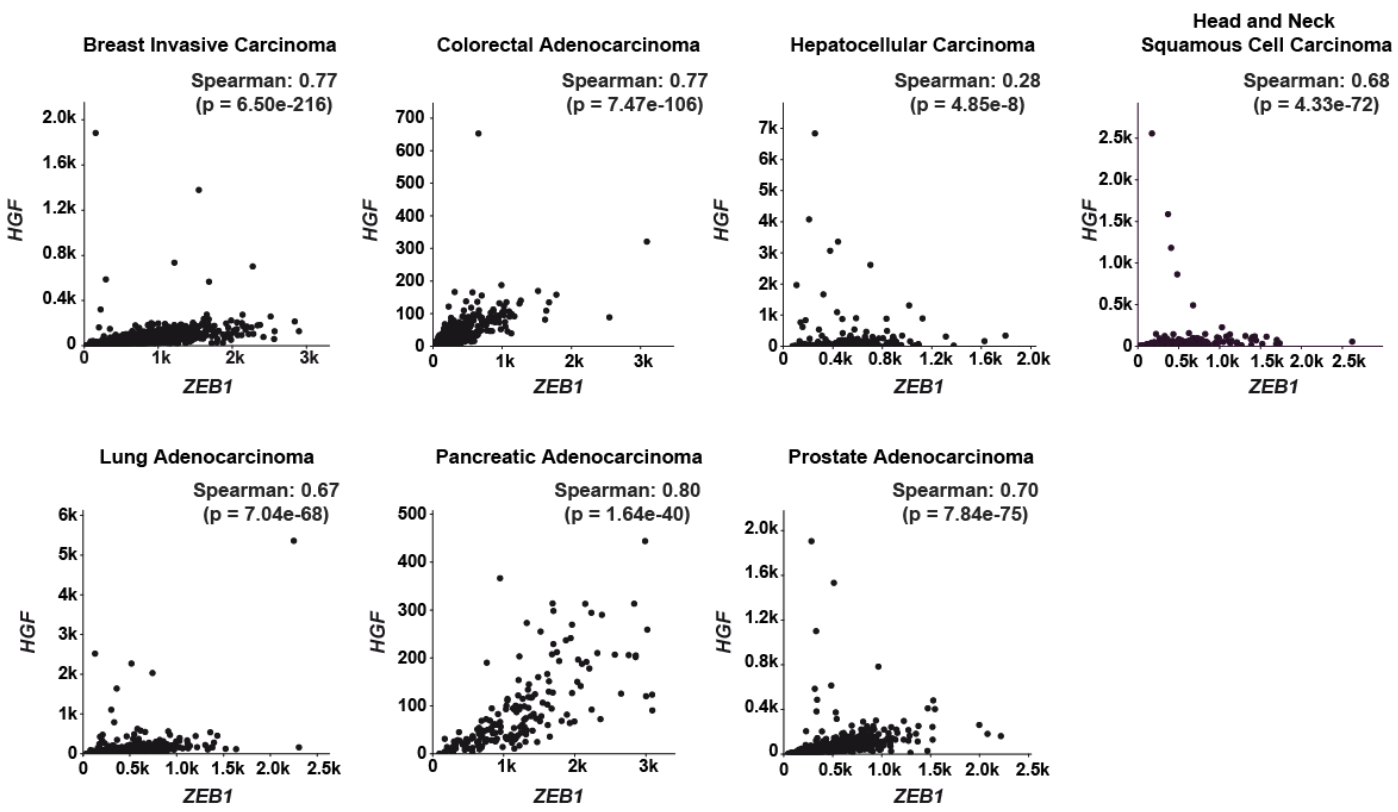

D

HGF promoter

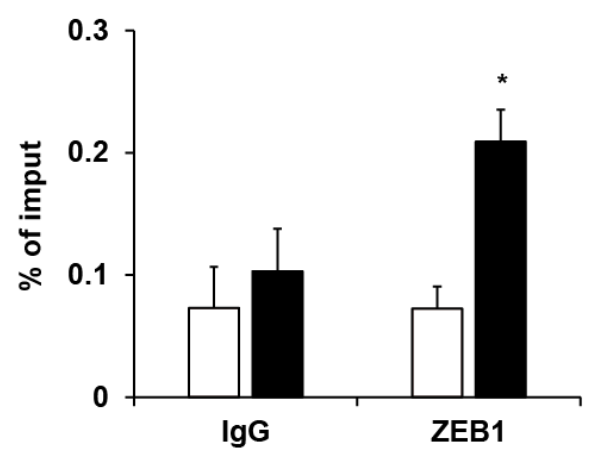

口HuCCT1 Control

- HuCCT1 Zeb1

Supplementary Figure 12. HGF expression correlates with that of ZEB1 in multiple human cancers and cell lines. A-C. Correlation of HGF gene expression with ZEB1 in Rennes and TCGA CCA cohorts (A), in cell lines from the Cancer Cell Line Encyclopedia (B) and in multiple cancers from the TCGA study (C), determined using Spearman's correlation analysis. C. Chromatin immunoprecipitation PCR (ChIP-PCR) experiment using primers targeting HGF promoter in HuCCT1 Control (white bars) and Zeb1 (black bars) cells. 
A

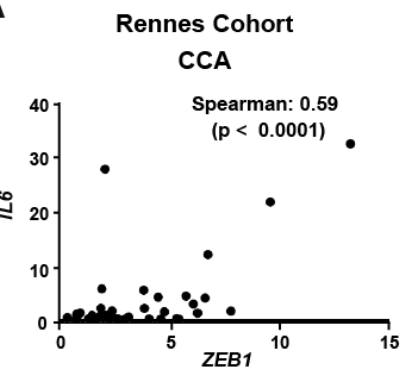

TCGA, PanCancer Atlas CCA

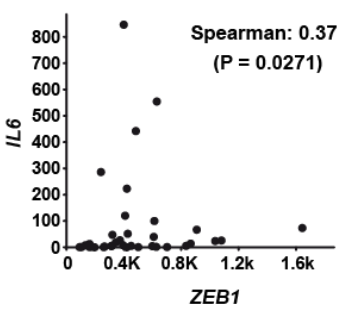

B (Cancer Cell Line Encyclopedia)

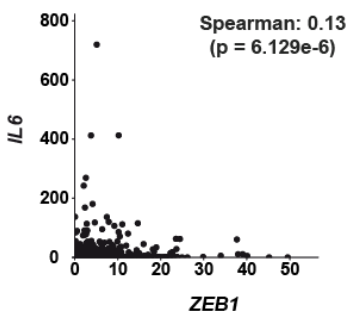

C (TCGA, PanCancer Atlas)
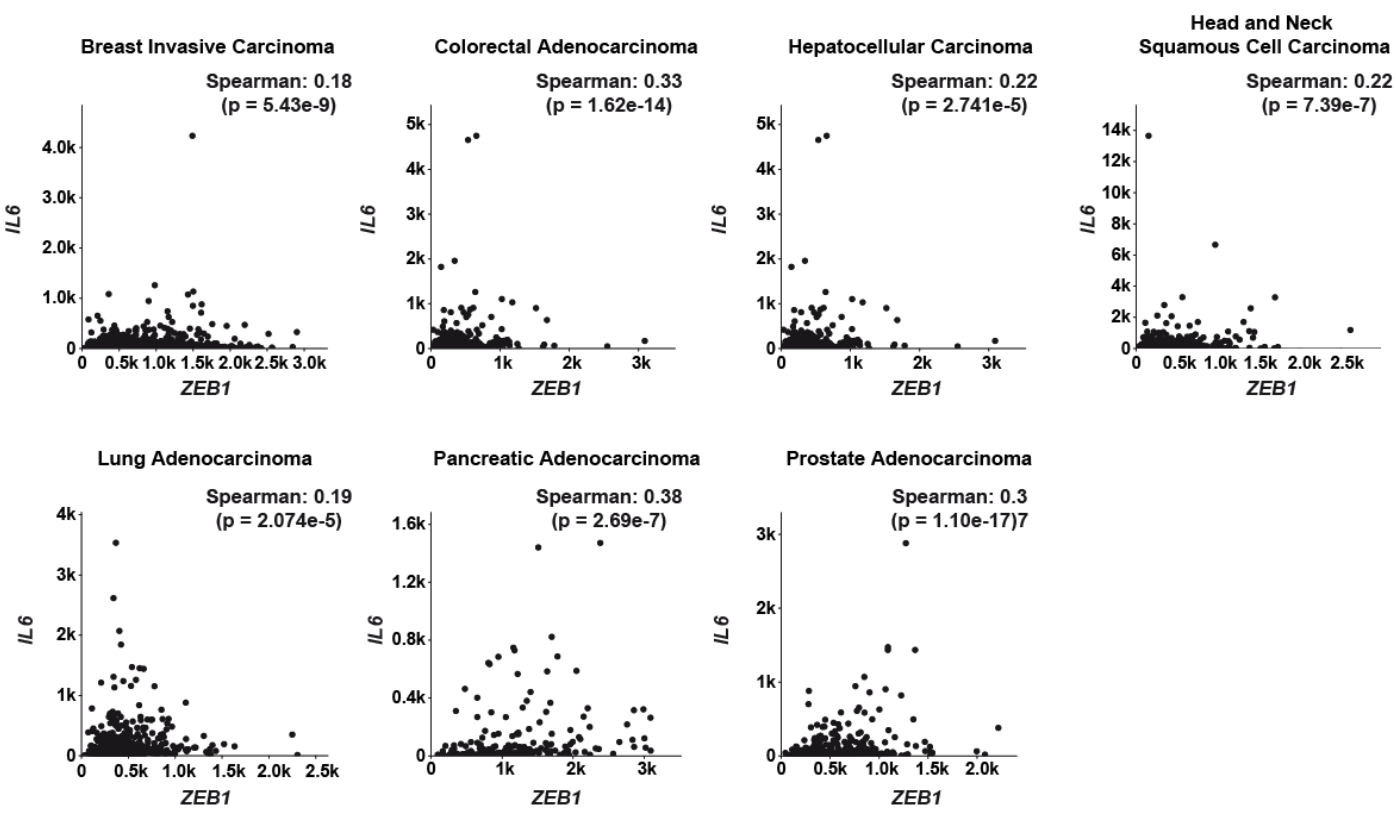

Supplementary Figure 13. IL6 expression correlates with that of ZEB1 in multiple human cancers and cell lines. A-C. Correlation of IL6 gene expression with ZEB1 in Rennes and TCGA CCA cohorts (A), in cell lines from the Cancer Cell Line Encyclopedia (B) and in multiple cancers from the TCGA study (C), determined using Spearman's correlation analysis. 
A

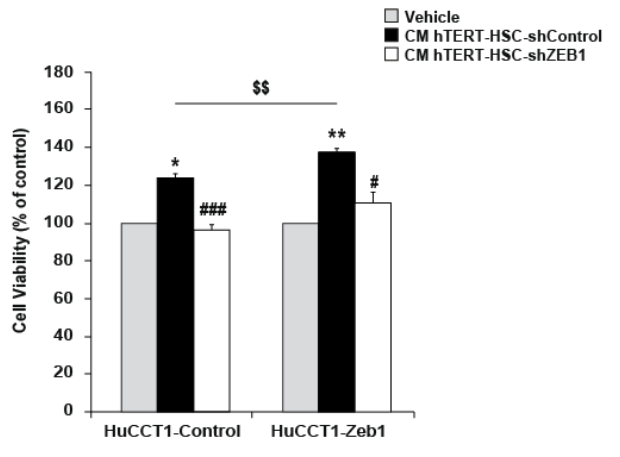

B

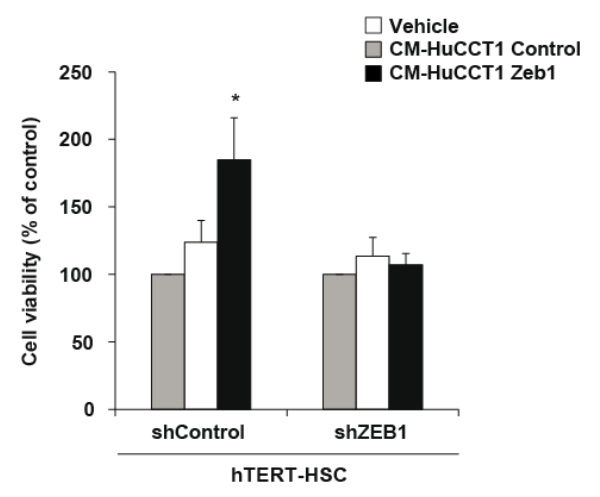

C

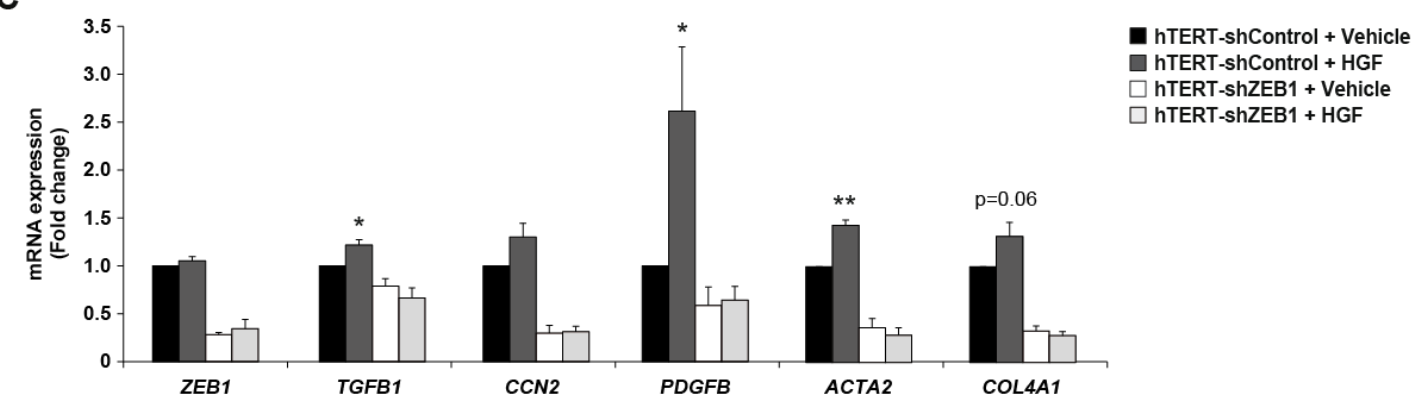

Supplementary Figure 14. A. Effect of conditioned media (CM) from hTERT-HSC shControl or shZEB1 cells on the viability of HuCCT1 Control and Zeb1 cells. B. Effect of CM from HuCCT1 Control and Zeb1 on the viability of hTERT-HSC shControl or shZEB1 cells. C. Effect of HGF on the expression of ZEB1, TGFB1, CCN2, PDGFB, ACTA2 and COL4A1 in hTERT-HSC shControl and shZEB1. mRNA expression was analyzed by RT-QPCR. Values are expressed as means \pm SEM from at least 4 independent cultures. ${ }^{*}, p<0.05 ;{ }^{*}, p<0.01$, as compared with Control cells (hTERTshControl + vehicle). *, $p<0.05 ;{ }^{* *}, p<0.01$; as compared with vehicle. \#, $p<0.05$; \#\#\#, $p<0.001$, as compared with CM from hTERT-HSC shControl. $\$ \$, p<0.01$ as compared with HuCCT1 Control cells. 


\section{A-MET}

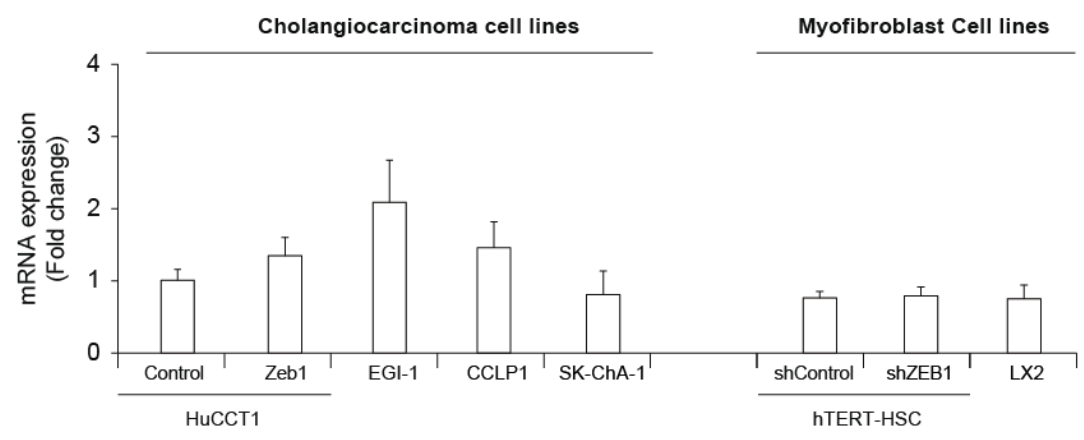

\section{B-IL6R}

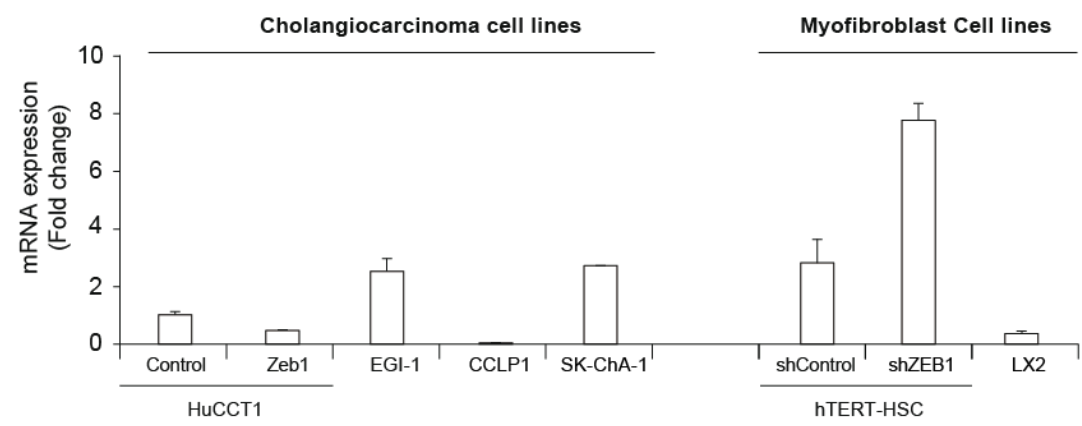

\section{C-IL6ST}

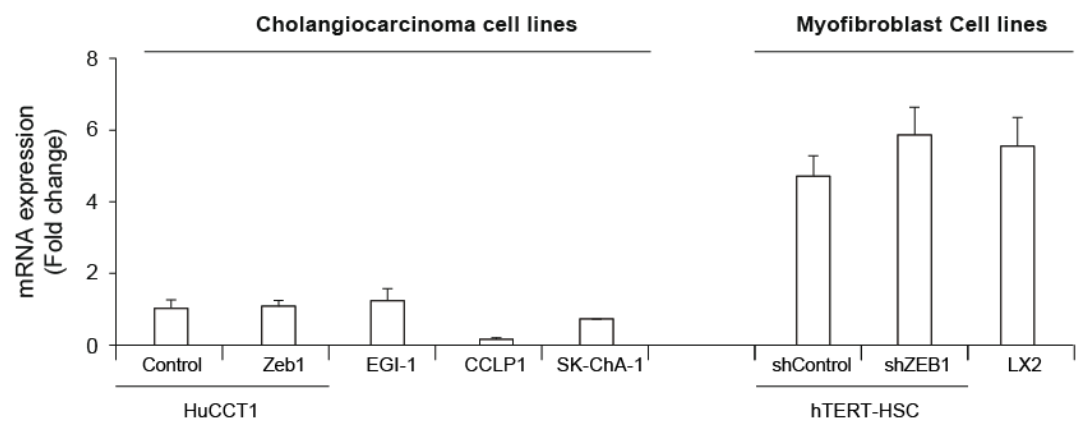

Supplementary Figure 15. mRNA expression of HGF receptor, MET, and of IL6 receptors, IL6R and IL6ST (GP130) in human CCA and hepatic myofibroblast cell lines. Expression is determined by RT-QPCR and values are expressed as means \pm SEM from 4 independent cultures. 
A

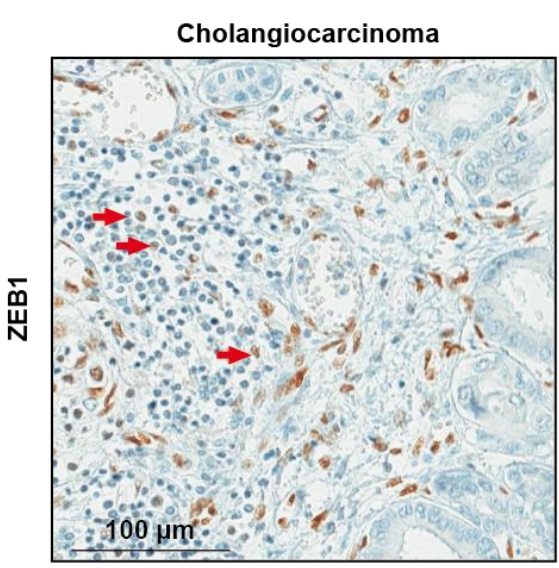

C
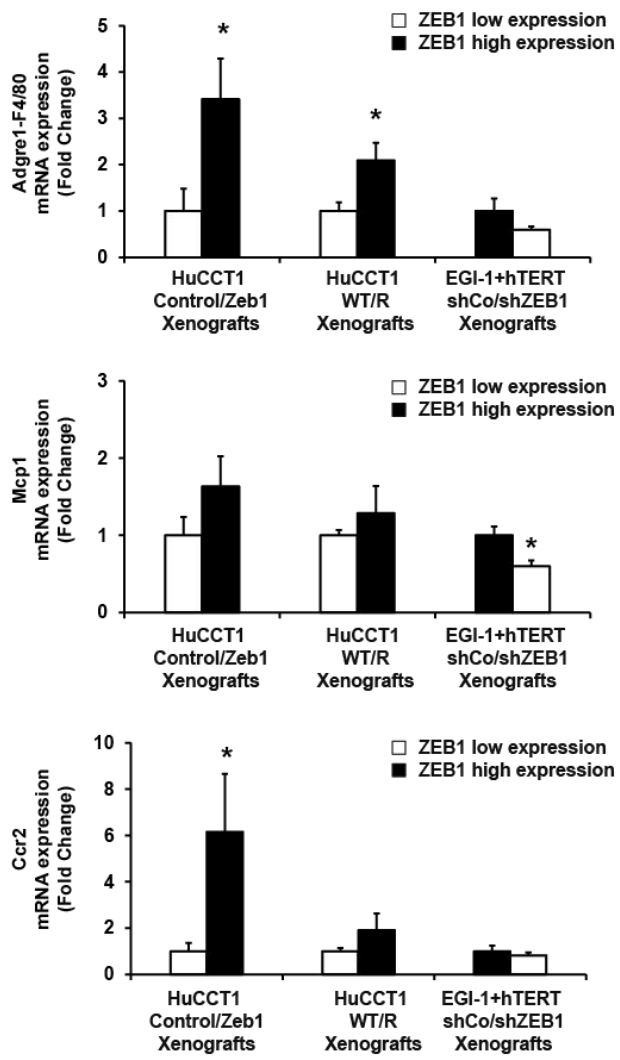

B

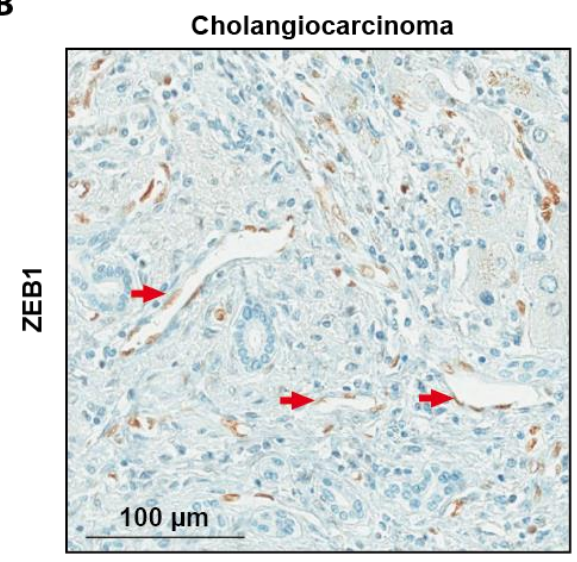

。
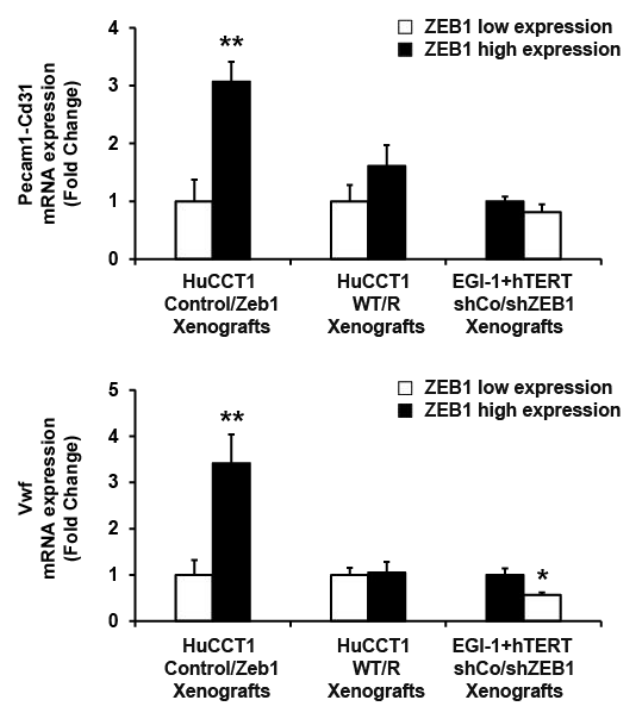

Supplementary Figure 16. A-B. Stromal cells other than CAF express ZEB1 in human intrahepatic CCA. A-B. Representative IHC staining of ZEB1, showing positive ZEB1 staining in immune cells $(A)$ and endothelial cells $(B)$. Red arrows point to either immune cells (A) or endothelial cells (B) expressing ZEB1. Scale: $100 \mu \mathrm{m}$. C-D. ZEB1 expression in tumor cells modifies other stromal cells than CAF in murine model of CCA. mRNA expression of murine Adgre (F4/80), Mcp1, Ccr2 (macrophage markers) (C), Pecam-1 (CD31) and Vwf (endothelial markers) (D), in xenografts from mice bearing HuCCT1 Control and Zeb1, HuCCT1 WT and R and the combination of EGl-1 plus hTERT-HSC-shControl and shZeb1 cells, analyzed by RT-QPCR. Values are expressed as means \pm SEM from 5 independent animals. ${ }^{*}, p<0.05 ;{ }^{* *}, p<0.01$, as compared with ZEB1 low expression. 
A

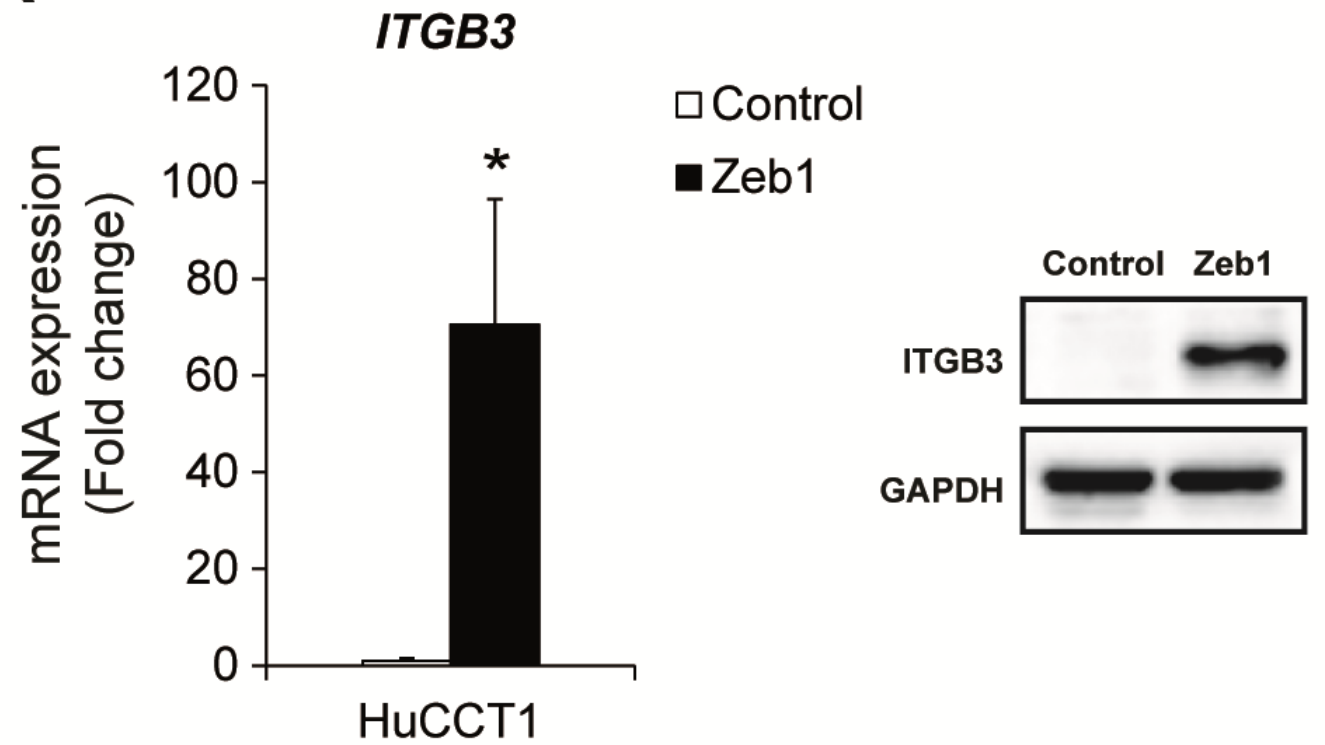

B

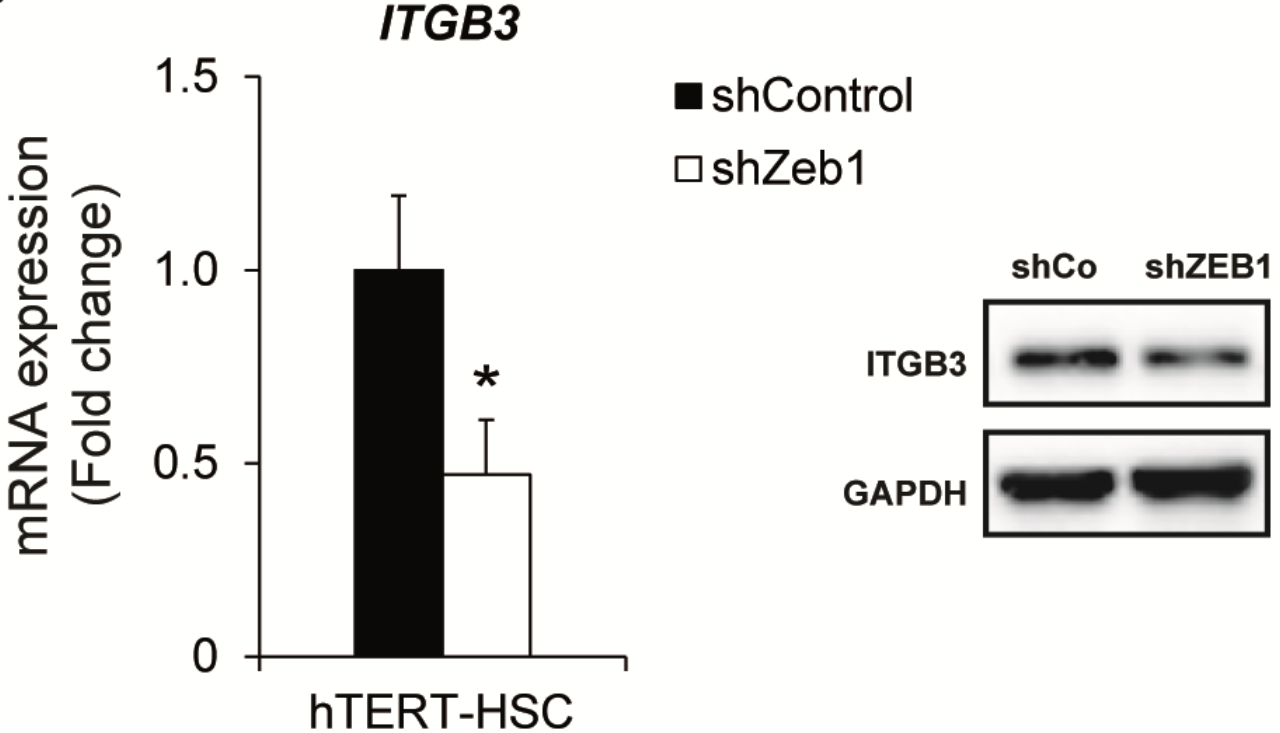

Supplementary Figure 17. A-B. Changes in ITGB3 (Integrin beta 3, also known as CD61) mRNA and protein expression in HuCCT1 Control and Zeb1 (A), and in hTERTHSC-shControl and shZEB1 (B), as determined by RT-QPCR and western blot. Results are expressed as means \pm SEM from 5 independent cultures. ${ }^{*}, \mathrm{p}<0.05$; as compared with Control or shControl cells. 


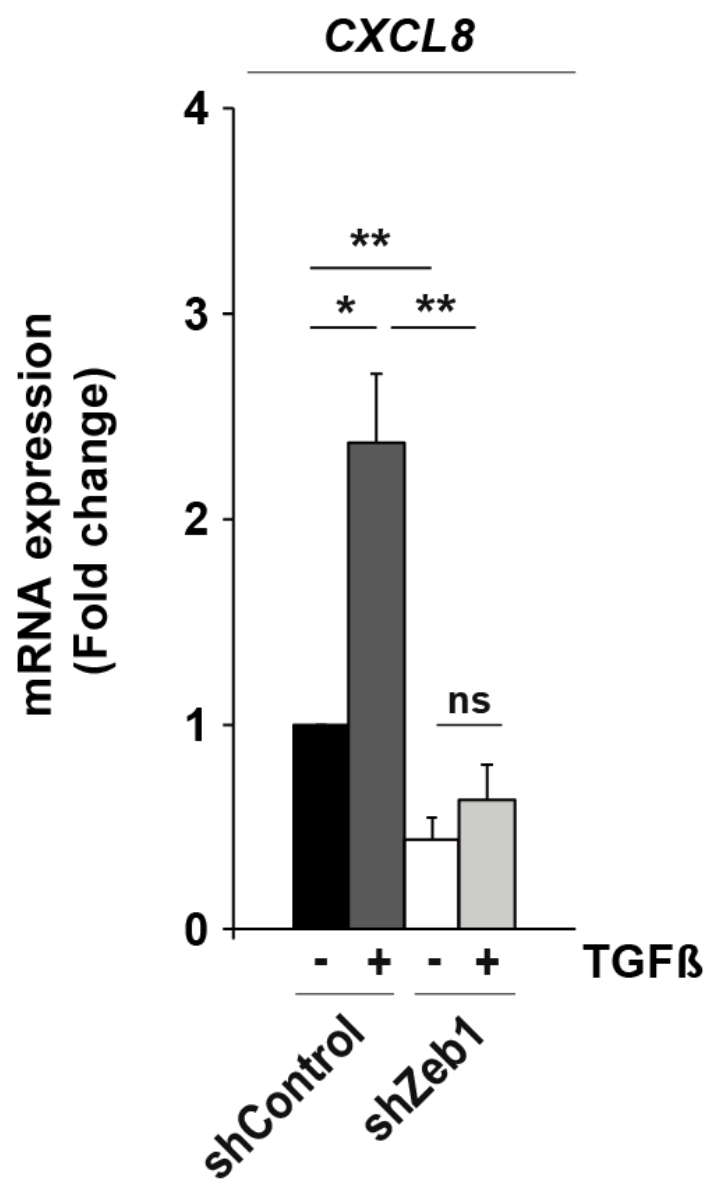

Supplementary Figure 18. ZEB1 in myofibroblasts impacts CXCL8 (IL8) expression. Changes in CXCL8 (IL8) in hTERT-HSC-shRNA-Control and shRNA-ZEB1 cells exposed to TGF- $\beta 110 \mathrm{ng} / \mathrm{ml}$ or the vehicle for $24 \mathrm{~h}$. Results are expressed as means \pm SEM from 5 independent cultures. ${ }^{*}, p<0.05 ;{ }^{* *}, p<0.01$, as indicated by lines. 